\title{
Geometric multiscale modeling of the cardiovascular system, between theory and practice
}

\author{
A. Quarteroni ${ }^{\mathrm{a}}$, A. Veneziani ${ }^{\mathrm{b}}$, C. Vergara $^{\mathrm{c}}$ \\ ${ }^{a}$ SB MATHICSE CMCS, EPFL, Lausanne, Switzerland \\ ${ }^{b}$ Department of Mathematics and Computer Science, Emory University, Atlanta (GA), United States \\ ${ }^{c}$ MOX, Dipartimento di Matematica, Politecnico di Milan, Italy
}

\begin{abstract}
This review paper addresses the so called geometric multiscale approach for the numerical simulation of blood flow problems, from its origin (that we can collocate in the second half of '90s) to our days. By this approach the blood fluid-dynamics in the whole circulatory system is described mathematically by means of heterogeneous problems featuring different degree of detail and different geometric dimension that interact together through appropriate interface coupling conditions.

Our review starts with the introduction of the stand-alone problems, namely the 3D fluidstructure interaction problem, its reduced representation by means of 1D models, and the so-called lumped parameters (aka 0D) models, where only the dependence on time survives. We then address specific methods for stand-alone 3D models when the available boundary data are not enough to ensure the mathematical well posedness. These so-called "defective problems" naturally arise in practical applications of clinical relevance but also because of the interface coupling of heterogeneous problems that are generated by the geometric multiscale process. We also describe specific issues related to the boundary treatment of reduced models, particularly relevant to the geometric multiscale coupling. Next, we detail the most popular numerical algorithms for the solution of the coupled problems. Finally, we review some of the most representative works - from different research groups - which addressed the geometric multiscale approach in the past years.

A proper treatment of the different scales relevant to the hemodynamics and their interplay is essential for the accuracy of numerical simulations and eventually for their clinical impact. This paper aims at providing a state-of-the-art picture of these topics, where the gap between theory and practice demands rigorous mathematical models to be reliably filled.
\end{abstract}

Keywords: Blood flow simulation, fluid-structure interaction, 1D models, lumped parameter models, geometric multiscale coupling.

\section{Introduction}

In the last two decades mathematical and numerical modeling of cardiovascular diseases has been progressively used to support medical investigations from basic research to clinical practice. This success of applied mathematics in such an important field of life sciences is not only due to the general improvement of high performance computing hardware, but also - and perhaps most importantly - to the development of more insightful and accurate mathematical models and effective 
numerical methods. As a matter of fact, these models have been specifically devised to include many features of medical interest in the simulation process with progressively more accuracy and precise quantification of the errors.

One of the aspects that engaged for several years bioengineers and mathematicians and promoted the development of special methodologies - that eventually found applications in other contexts - is the treatment of boundary conditions. This issue is particularly sensitive for the reliability of numerical solutions and it is particularly challenging for at least two reasons.

1. Lack of available data: the mathematical boundary might not be a real physical one. This is e.g. the case of the inlet and outlet of arteries "artificially chopped" for computational purposes. For this reason, a significant gap between data available in practice from measurements and boundary conditions required by the mathematical problem occurs systematically; more than in other engineering fields, either practical or ethical reasons prevent to obtain all the data that are required by the mathematical model.

2. Reciprocal influence of the local and systemic dynamics: circulation is a closed network of vessels featuring different properties (both geometrical and mechanical) in different regions, where local disturbances (induced for instance by a pathology or a surgery) may have a global impact. When setting up the simulation of a local vascular district, it is generally required to include this mutual influence; this eventually resorts to a proper boundary treatment, where the (artificial) boundaries represent in fact the interface between the local region of interest and the rest of the system.

The latter issue somehow justifies the introduction of the term "multiscale", since it basically stems from the coupling of dynamics acting on scales of centimeters (a single vascular district) and of meters (the entire network). However, since this term may assume different meaning in different engineering and modelling fields, it seems appropriate to specify that here with multiscale we mean the coupling of different length scales, so that we will use this term in combination with the adjective "geometric". While a local detailed hemodynamic analysis requires in general the accurate solution of fluid-structure interaction problems (blood and vascular walls), henceforth in the true 3D domain, quantitative investigations of the cardiovascular system have often been based on surrogate models featuring lower geometric dimensions. We recall the pioneering work by Otto Frank [77], followed up by the simulators of Nico Westerhof [222], based on the analogy of the circulatory network with electrical circuits. These are lumped parameter or - with a popular notation that follows from discarding an explicit dependence on any space dimension and that will be used extensively later on - 0D models. Even earlier (two centuries!) L. Euler proposed his equations for describing the motion of a fluid in elastic pipes, having in mind blood flow in arteries [60]. This system of equations has then provided the baseline for assembling mathematical models of several arterial segments, in each of them the axial dynamics is the only one retained, resorting to what we will denote as 1D models. Because of their hyperbolic nature, these models turned out to be particularly effective in predicting the pressure wave propagation along the arterial tree.

The two issues listed above turn out to be strictly related. In order to address point 2 above bioengineers looked for reliable boundary conditions for a district of interest by solving Westerhof-like 0D models to be prescribed in a specific district. Then, to solve the incompressible Navier-Stokes equations in that district, this naturally brought up the problem of defective data set, as for point 1. For example, a lumped parameter as well as 1D model can provide a flow rate incoming a district of interest. However a Navier-Stokes solver for that district requires the whole velocity field at the boundaries. A practical and popular approach consists in conjecturing an a priori velocity profile 
(typically a parabolic one) to be fitted with the flow rate available from the systemic model. Similar considerations hold for Neumann-like conditions such as those prescribing the traction or the pressure. However, the accuracy of these heuristic approaches may be sometimes questionable. A more sound mathematical approach was deemed in order to enhance both reliability and accuracy. Starting from the second half of 90's with the paper [179], this problem challenged several groups and led to many different ideas.

The purpose of this work is to critically review these topics in order to highlight the important impact that mathematically sound methods may have on the accuracy of the results. Nevertheless, we will include in our discussion also practical aspects that need to be considered when performing geometric multiscale simulations on real problems.

Moving from a brief description (Sect. 2) of the different models that can be used in a standalone fashion to describe the circulation with a different level of detail (3D, 1D or 0D), we consider more specifically the issues related to their boundary treatment in Sect. 3. While for the 3D problem we need to consider how to fill the gap between insufficient available data and a complete data set, for 1D problems the treatment of the boundary requires special techniques to avoid numerical artifacts in computing the pressure wave propagation. Finally, for 0D models the concept of "boundary" is actually inappropriate, since the model reduction drops the explicit space dependence. However, in view of coupling dimensionally heterogeneous models, we need to address how data at the interface of the lumped parameter compartment can be spatially localized. In Sect. 4 we address extensively the coupling of the different models that leads to a "geometric multiscale" description, whereas we will address different approaches for the numerical solution of the dimensionally heterogeneous problems in Sect. 5. In Sect. 6 we provide an annotated review of selected works to outline significant contributions of the literature over the last two decades. Conclusions and perspectives follow in Sect. 7 .

\section{Stand-alone models: fluid, structure and their interaction}

In this section, we start from the classical 3D model for fluid-structure interaction in hemodynamics. We then address the 1D and finally 0D models. Each of these models is standing alone; the analysis of coupling will make the subject of next sections. We necessarily limit to a brief introduction to this vast and still active field of research.

\subsection{The 3D model}

\subsubsection{Modeling blood, vascular wall and their interaction}

We start considering a 3D high fidelity description of blood flowing in a vessel of interest, the vascular wall deformation, and their interaction (fluid-structure interaction - FSI).

It is worth mentioning that many vascular diseases affect large and medium sized arteries. In such districts, blood is modeled by means of the Navier-Stokes (NS) equations for incompressible homogeneous Newtonian fluids [163, 200, 201, 71]. For non-Newtonian rheological models necessary to describe some specific flow processes, such as clotting, or sickle cell diseases, or more generally flow in capillaries, we refer, e.g., to [185].

As for the structure problem, we assume the arterial wall to obey a (possibly nonlinear) finite elastic law relating stress to strain in the arterial tissue. This is clearly a simplification of the indeed far more complex behavior of arterial walls $[100,101]$ that however we postulate for the 

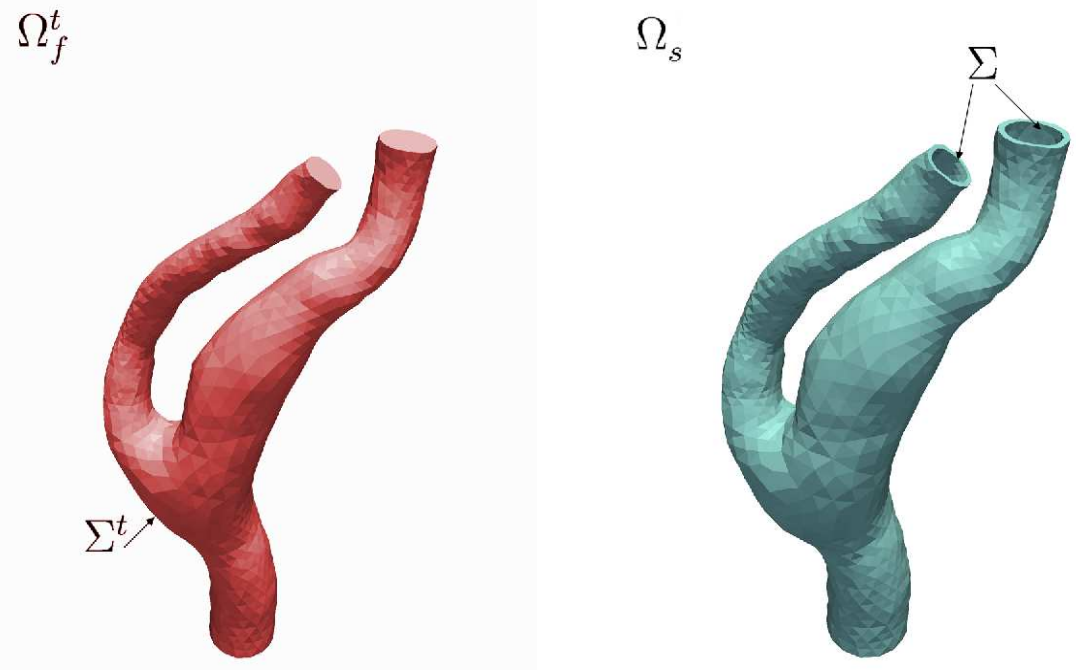

Figure 1: Representation of the two components of the FSI problem: fluid domain on the left, structure domain on the right. The fluid domain - here we illustrate a carotid artery - has been reconstructed from MRI images (Courtesy of Dr. M. Domanin, Fondazione IRCSS Cà Granda, Ospedale Maggiore Policlinico, Milan, Italy), whereas the structure domain has been obtained by extrusion of the fluid one - see Sect. 2.1.3.

sake of simplicity. In more realistic settings, strain is function of the stress but also of the loading history [79].

For the mathematical formulation of the problem, we find convenient to write the fluid equations with respect to an Eulerian frame of reference, and we denote by $\Omega_{f} \subset \mathbb{R}^{3}$ the time-varying arterial lumen (see Figure 1, left), while the structure problem is usually written in a reference or material domain $\widetilde{\Omega}_{s} \subset \mathbb{R}^{d}$ using a Lagrangian framework. For any $t>0$ the spatial domain $\Omega_{s}$ (depicted in Fig. 1, right) is then regarded as the image of $\widetilde{\Omega}_{s}$ by a proper Lagrangian map $\mathcal{L}: \widetilde{\Omega}_{s} \rightarrow \Omega_{s}$. We use the abridged notation $\widetilde{g}=g \circ \mathcal{L}$ to denote in $\widetilde{\Omega}_{s}$ any function $g$ defined in the current solid configuration $\Omega_{s}$. The interface between the fluid and the structure domains at time $t>0$ is denoted by $\Sigma$.

We denote by $\boldsymbol{F}=\nabla \boldsymbol{x}$ the deformation tensor, the gradient being taken with respect to the reference space coordinates. Correspondingly, $J=\operatorname{det}(\boldsymbol{F})$ represents the change of volume between the reference and the current configurations.

Under all the assumptions stated above, we eventually write the 3D fluid-structure interaction problem as follows. Find, at each time $t \in(0, T]$, fluid velocity $\boldsymbol{u}$, fluid pressure $p$ and structure 
displacement $\boldsymbol{\eta}$, such that

$$
\begin{array}{lc}
\rho_{f} \frac{\partial \boldsymbol{u}}{\partial t}+\rho_{f}(\boldsymbol{u} \cdot \nabla) \boldsymbol{u}-\nabla \cdot \boldsymbol{T}_{f}(\boldsymbol{u}, p)=\boldsymbol{f}_{f} & \text { in } \Omega_{f} \\
\nabla \cdot \boldsymbol{u}=0 & \text { in } \Omega_{f} \\
\boldsymbol{u}=\frac{\partial \boldsymbol{\eta}}{\partial t} & \text { on } \Sigma, \\
\boldsymbol{T}_{s}(\boldsymbol{\eta}) \boldsymbol{n}-\boldsymbol{T}_{f}(\boldsymbol{u}, p) \boldsymbol{n}=\mathbf{0} & \text { on } \Sigma, \\
\rho_{s} \frac{\partial^{2} \widetilde{\boldsymbol{\eta}}}{\partial t^{2}}-\nabla \cdot \widetilde{\boldsymbol{T}}_{s}(\widetilde{\boldsymbol{\eta}})=\boldsymbol{f}_{s} & \text { in } \widetilde{\Omega}_{s} .
\end{array}
$$

In the previous problem $\rho_{f}$ and $\rho_{s}$ are the fluid and structure densities respectively, $\boldsymbol{f}_{f}$ and $\boldsymbol{f}_{s}$ are volumetric forces acting in the two domains (e.g. corresponding to gravity or muscle forces in the walls - quite often these contributions can be neglected),

$$
\boldsymbol{T}_{f}(\boldsymbol{u}, p)=-p \boldsymbol{I}+\mu\left(\nabla \boldsymbol{u}+(\nabla \boldsymbol{u})^{T}\right)
$$

is the fluid Cauchy stress tensor with $\mu$ the blood viscosity. As we consider only Newtonian rheology here, $\mu$ is assumed to be constant. In addition, $\boldsymbol{n}$ denotes the outward unit normal from the structure domain and the first Piola-Kirchhoff tensor $\widetilde{\boldsymbol{T}}_{s}(\widetilde{\boldsymbol{\eta}})$ and the Cauchy tensor $\boldsymbol{T}_{s}(\boldsymbol{\eta})$ are such that $\widetilde{\boldsymbol{T}}_{s}=J \boldsymbol{T}_{s} \boldsymbol{F}^{-T}$. For an hyperelastic material, the first Piola-Kirchhoff stress tensor is obtained by differentiating a suitable Strain Energy Density Function (SEDF) $\Theta$ such that $\widetilde{\boldsymbol{T}}_{s}=\frac{\partial \Theta}{\partial \boldsymbol{F}}$. For arteries, several non-linear elastic energy functions have been proposed. A simple choice is provided by the Saint-Venant-Kirchhoff material, in which case the first Piola-Kirchhoff tensor is given by

$$
\widetilde{\boldsymbol{T}}_{s}=\frac{E \nu}{(1+\nu)(1-2 \nu)}\left(\operatorname{tr}\left(\boldsymbol{F}^{T} \boldsymbol{F}\right)-3\right) \boldsymbol{F}-\frac{E}{2(1+\nu)} \boldsymbol{F}+\frac{E}{2(1+\nu)} \boldsymbol{F F}^{T} \boldsymbol{F},
$$

where $E$ is the Young modulus and $\nu$ the Poisson modulus. To take into account the stiffening increment for large displacements due to the collagen, an exponential law is often used

$$
\widetilde{\boldsymbol{T}}_{s}=G J^{-2 / 3}\left(\boldsymbol{F}-\frac{1}{3} \operatorname{tr}\left(\boldsymbol{F}^{T} \boldsymbol{F}\right) \boldsymbol{F}^{-\mathrm{T}}\right) e^{\gamma\left(J^{-\frac{2}{3}} \operatorname{tr}\left(\boldsymbol{F}^{T} \boldsymbol{F}\right)-3\right)}+\frac{\kappa}{2}\left(J-1+\frac{1}{J} \ln (J)\right) J \boldsymbol{F}^{-\mathrm{T}},
$$

where $\kappa$ is the bulk modulus, $G$ the shear modulus, and $\gamma$ characterizes the stiffness of the material for large displacements $[78,100,182]$. More complex models account for the collagen fibers' orientation, identified by the unit vector $\boldsymbol{m}$. In this case, a popular approach relies on separating the isotropic behavior of the ground substance given by elastin, described by a neo-Hookean model, from the anisotropic response due to the collagen fibers, obtaining

$$
\widetilde{\boldsymbol{T}}_{s}=k_{1} \boldsymbol{F} \boldsymbol{F}^{T}+k_{2}(I-1) e^{\gamma(I-1)^{2}}(\boldsymbol{F} \boldsymbol{m}) \otimes(\boldsymbol{F} \boldsymbol{m}),
$$

with $I=\boldsymbol{m} \cdot\left(\boldsymbol{F}^{T} \boldsymbol{F} \boldsymbol{m}\right)$ being an invariant of the system and $k_{1}, k_{2}$ suitable material parameters [101]. More complete laws also account for the symmetrical helical arrangement of the collagen fibers, with directions $\boldsymbol{m}$ and $\boldsymbol{m}^{\prime}$ lying in the tangential plane of the artery [101]. The arterial tissue is sometimes considered as incompressible [44]. In this case, one has to enforce the constraint 
$\operatorname{det} J=1$ and in the related Cauchy stress tensor the term $-p_{s} \boldsymbol{I}$ is added, $p_{s}$ being the hydrostatic pressure (which plays the role of Lagrange multiplier of the incompressibility constraint).

The matching conditions enforced at the FS interface follow from the continuity of velocities (1c) (kinematic condition) and the continuity of tractions (1d) (dynamic condition - NOTICE THAT HERE "DYNAMIC" HAS BEEN USED AS OPPOSED TO "KINEMATIC") .

Finally, problem (1) is completed by boundary conditions at $\partial \Omega_{f} \backslash \Sigma$ and $\partial \widetilde{\Omega}_{s} \backslash \widetilde{\Sigma}$, and by initial conditions on $\boldsymbol{u}, \boldsymbol{\eta}$ and $\frac{\partial \boldsymbol{\eta}}{\partial t}$. Boundary conditions typically prescribe:

- for the fluid subproblem, the upstream velocity $\boldsymbol{u}_{u p}$ on the proximal boundaries and absorbing traction conditions $\boldsymbol{T}_{f} \boldsymbol{n}=\mathbf{h}$ on the distal boundaries, $\boldsymbol{h}$ being a suitable function [150];

- for the structure subproblem, either $\boldsymbol{\eta}=\mathbf{0}$ (fixed boundary) or $\boldsymbol{\eta} \cdot \boldsymbol{n}=0$ together with $\left(\boldsymbol{T}_{s} \boldsymbol{n}\right) \cdot \boldsymbol{\tau}=\mathbf{0}$, $\tau$ being the unit tangential directions (displacement allowed in the tangential direction).

Other conditions may be prescribed if patient-specific measured data are available. However as pointed out in the Introduction - measures seldom provide a complete data set to be used in the computation and a preprocessing step is required as we will illustrate in Sect. 3.

Boundary conditions at the external lateral boundary of the structure account for the effect of the tissues surrounding the artery. In [140], an algebraic law is proposed to mimic an elastic behavior of this tissue. This law is meant at representing the action of these tissues by independent springs characterized by an elastic space dependent coefficient $\alpha_{S T}$ (ST stands for "surrounding tissues"). This yields the following Robin boundary condition

$$
\alpha_{S T} \widetilde{\boldsymbol{\eta}}+\widetilde{\boldsymbol{T}}_{s}(\widetilde{\boldsymbol{\eta}}) \widetilde{\boldsymbol{n}}=P_{e x t} \widetilde{\boldsymbol{n}}, \quad \text { on } \widetilde{\Sigma}_{e x t},
$$

where $\Sigma_{\text {ext }}$ is the external lateral surface and $P_{\text {ext }}$ the external pressure. For tuning $\alpha_{S T}$, we refer the reader to $[126,49]$.

Under several regularity assumptions, these data may guarantee well posedness to the coupled fluid-structure problem, see e.g. $[18,86,35,128]$ for a comprehensive description of this topic.

\subsubsection{Numerical discretization}

Numerical approximation of (1) demands an appropriate discretization of time as well as space variables. One of the challenging aspects here is the movement of the domain, both for the fluid and the solid. For the structure, deformations are in general small enough so that a purely Lagrangian description is a viable option. On the contrary, for the fluid we need to use a Lagrangian description of the fluid-structure interface and an Eulerian description of the proximal/distal boundaries. As pointed out in the Introduction, these are artificial portions of the boundary and their location does not follow the fluid displacement. This hybrid situation led to the introduction of the so-called Arbitrary Lagrangian-Eulerian (ALE) formulation [104, 58]. With this approach the displacement field at the boundary is arbitrarily extended into the domain. For instance, a harmonic lifting (i.e. the displacement computed by solving a Laplace problem) is a popular choice. This provides a convenient yet non-inertial frame of reference where to write the Navier-Stokes equations (ALE formulation). In this framework, the solution of the fluid and structure problem is supplemented by the solution of the lifting (hereafter called "geometric coupling problem"). Different methods can be used for the solution of the FSI plus geometric coupling problem. Time discretization can be obtained by standard finite difference procedures. Among the others, we mention Backward 
Difference Formulas (BDF), successfully adopted for both fluid and structure problems. Alternatively, the $\vartheta$-method for fluid and Newmark schemes for the structure are successfully used e.g. in [147]. For the space discretization, finite elements and finite volumes are the most popular strategies. Notice however that the movement of the domain makes the accuracy analysis of the overall procedure quite challenging as the interplay between space and time accuracy of the discretization of the fluid, structure and geometric coupling problems is not trivial.

At the algorithmic level, after a suitable treatment of the geometric coupling (either implicit applying, e.g., the Newton method [148] or explicit by means of extrapolation from previous time steps [62]), the FSI problem may be solved by monolithic as well as segregated approaches. In the former case, the complete non-linear system arising after the space discretization is assembled and solved with a suitable preconditioned Krylov [96, 15], domain-decomposition [50, 57] or multigrid $[82,13]$ methods. In the partitioned case the successive solution of the fluid and solid subproblems in an iterative framework is carried out (see, e.g., $[45,62,11,53,9,117,148]$ ). In this case, the schemes feature in general poor convergence properties due to the added mass effect, that predicts a breakdown of performances when the values of the densities of fluid and structure are close as it happens in hemodynamics $[45,76,10,85,151,84]$. For the sake of COnCRETEness, we Refer here to Finite Element discretization for the space dependence and Finite DiffERENCES FOR THE TIME DEPENDENCE. Alternatively, one could consider space-time finite elements, see, e.g., [203, 17], or the iso-geometric analysis, see [15, 16].

It is worth noting that for problems related to the movement of structures floating in incompressible fluids, a successful approach is the so-called Immersed Boundary Method originated by the work of C. Peskin $[167,37]$.

Recent introductions to the numerical approximation of FSI problems can be found in [61, 17].

\subsubsection{Further developments and comments}

Modeling the structure as a 2D membrane. For the sake of simplification, the structure may be modelled as a 2D membrane whose position in space at any time exactly coincides with the FS interface $\Sigma$. In this case, only the radial displacement $\eta_{r}$ is considered, and a possible mathematical representation is given by the generalized string model [177]:

$$
\rho_{s} H_{s} \frac{\partial^{2} \widetilde{\eta}_{r}}{\partial t^{2}}-\nabla \cdot\left(P \nabla \widetilde{\eta}_{r}\right)+\beta H_{s} \widetilde{\eta}_{r}=\widetilde{f}_{s} \text { at } \widetilde{\Sigma},
$$

where the manifold $\widetilde{\Sigma}$ represents the reference membrane configuration, $H_{s}$ the structure thickness, tensor $P$ accounts for shear deformations and, possibly, for prestress, $\beta(\boldsymbol{x})=\frac{E}{1-\nu^{2}}\left(4 \rho_{1}^{2}-2(1-\nu) \rho_{2}\right)$, where $\rho_{1}(\boldsymbol{x})$ and $\rho_{2}(\boldsymbol{x})$ are the mean and Gaussian curvatures of $\widetilde{\Sigma}$, respectively, [150], and $f_{s}$ the forcing term. The previous model is derived from the equations of the linear infinitesimal elasticity (Hooke law) under the assumptions of small thickness, plane stresses, and negligible elastic bending terms. To account for the effect of the surrounding tissue, the term $\beta$ in (4) needs to be properly modified. For example, in the case of an elastic tissue as in (3), we need to substitute $\beta$ with $\widehat{\beta}=\beta+\alpha_{S T}$, with $\alpha_{S T}$ the elastic coefficient of the tissue.

In the particular case where $\widetilde{\Sigma}$ is the lateral surface of a cylinder and any dependence on the circumferential coordinate is discarded, model (4) reduces to

$$
\rho_{s} H_{s} \frac{\partial^{2} \widetilde{\eta}_{r}}{\partial t^{2}}-k G H_{s} \frac{\partial^{2} \widetilde{\eta}_{r}}{\partial z^{2}}+\frac{E H_{s}}{\left(1-\nu^{2}\right) R_{0}^{2}} H_{s} \widetilde{\eta}_{r}=f_{s} \quad \text { at } \widetilde{\Sigma}
$$


$k$ being the Timoshenko correction factor, $G$ the shear modulus, $R_{0}$ the cylinder radius, and $z$ the axial coordinate. Often, in the latter case, also a visco-elastic term of the form $\gamma_{v} \frac{\partial^{3} \eta_{r}}{\partial^{2} z \partial t}$ is added, with $\gamma_{v}$ a suitable visco-elastic parameter [177].

When (4) is coupled with the fluid equations (1a)-(1b), possible matching conditions read

$$
\begin{array}{ll}
\boldsymbol{u} \cdot \boldsymbol{n}=\frac{\partial \eta_{r}}{\partial t} & \text { at } \Sigma, \\
\boldsymbol{T}_{f}(\boldsymbol{u}, p) \boldsymbol{n} \cdot \boldsymbol{n}=f_{s} & \text { at } \Sigma .
\end{array}
$$

In fact, the coupling occurs only in the radial direction, so that we have to complete the conditions at $\Sigma$ for the fluid problem by prescribing tangential information, e.g., homogeneous Dirichlet or Neumann data.

In [64] an effective formulation to solve the FSI problem with a membrane structure is proposed, whereas in [46] the accuracy of the FSI-membrane problem is discussed in comparison to full 3D3D/FSI results. For the Hooke law, the wall shear stresses computed with the two FSI models are in good agreement for a distal arterial tract such as a femoropopliteal bypass. Instead, when larger displacements are considered such as in the ascending aorta, the discrepancies between the two FSI models increase as high as more than $10 \%$. At a theoretical level, it is interesting to note that the assumption of purely radial displacement not only simplifies the computation, but also improves the properties of the associated numerical ALE approximation [149].

Geometrical reconstruction of $3 D$ domains. To accurately simulate the hemodynamics and fluidstructure interaction in patient-specific settings, the individual morphology needs to be retrieved from available images. This is possible in different ways, depending on the source of the data (Computed Tomography, Magnetic Resonance, Intravascular Ultrasound, Optical Coherence Tomography, Positron Emission Tomography, to mention a few) [202]. This attains to the field of image and geometric processing and the parallel progress of imaging devices as well as computational geometry techniques led to terrific advancement in the field. However, the reconstruction of vascular walls may be still troublesome as some imaging techniques can reconstruct the interface $\Sigma$ (and thus the corresponding lumen $\Omega_{f}$ ). This can be listed as another example of "lack of data" and practical gap between data needed and actually available in cardiovascular mathematics. Black Blood MRI can actually provide wall reconstruction (see e.g. [5]), but when this is not possible a reasonable approach is to extrude the interface $\Sigma$ along the outward unit vector by postulating a reliable function that specifies the vessel thickness in the different regions of a district of interest. This is the case reported in Fig. 1 for a carotid artery. In this example, thickness was assumed to be constant.

EXPERIMENTAL EVIDENCE CLEARLY HIGHLIGHTS THAT ARTERIAL TISSUE FEATURES AN INTRINSIC PRE-LOAD, CORRESPONDING TO A NONTRIVIAL REST CONDITION. THE LATTER SHOULD Be included in the Constitutive law of the Structure. Pre-LoAd CAN Be included ei-

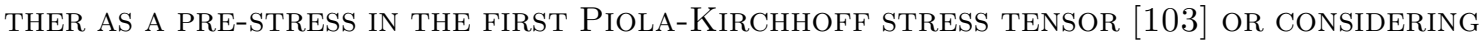
A MODIFIED UPDATED LAgRANGIAN FORMULATION [83]. UNFORTUNATELY, PRE-LOAD QUANTITATIVE DETERMiNATION IS DIFFICULT, SINCE AUTOPTIC SPECIMENS DO NOT REFLECT THE REAL "IN VIVO" CONDition IN GENERAL. NumERICAL ESTIMATION TECHNiques CAN BE CONSIDERED, BASED ON THE SOLUTION OF INVERSE ELASTOGRAPHY-LIKE PROBLEMS (SEE, E.G., $[52,127]$ ) OR the ReCONSTRUCtion of the Zero-STRESS GeOMETRY (SEe $[39,121]$ ). To THIS AIM, RECENT IMAGING DEVICES ABLE OF PERFORMING 4D ACQUISITIONS ARE EXPECTED 
TO PLAY A MAJOR ROLE IN THE NEXT YEARS. IN FACT, THE REGISTRATION PROCESS MAY ALLOW THE DISPLACEMENT RETRIEVAL AND EVENTUALLY THE PRELOAD CAN BE ESTIMATED BY SOLVING AN INVERSE FLUID-STRUCTURE INTERACTION PROBLEM (SIMILAR TO WHAT PROPOSED IN [162] FOR ARTERIAL COMPLIANCE, SEE BELOW). IN THIS REVIEW PAPER, FOR THE SAKE OF SIMPLICITY WE DO NOT INCLUDE THIS ASPECT IN THE FOLLOWING ANALYSIS. HOWEVER, MODELS AND METHODS DESCRIBED HEREAFTER CAN BE PROMPTLY EXTENDED TO CONSTITUTIVE LAWS INCLUDING PRE-LOAD. FOR A RECENT NUMERICAL STUDY ABOUT THE EFFECT OF IGNORING THE PRELOAD IN REAL GEOMETRIES, WE REFER THE INTERESTED READER TO [26]. For a comprehensive introduction to image and geometric processing in vascular hemodynamics, see $[3]$.

Parameter estimation for the constitutive laws. To estimate the values of the parameters in the coupled problem (1)-(3) several experimental methods have been proposed so far, as the pulse wave velocity method, based on measuring the rate of propagation of flow waves coming form the heart, to estimate vessels compliance [36]. Alternatively, mathematical approaches based on the solution of inverse problems have been recently proposed, such as a variational approach based on the minimization of a suitable functional [162] and an approach based on the Kalman filter $[22,155]$. For a review of estimate procedures in fluid-structure interaction see [19].

\subsection{The $1 D$ model}

Numerical modeling of the whole cardiovascular system by means of 3D models is currently out of reach because of the complexity of the computational domain, that would require the acquisition and reconstruction of thousands (or even more) vessels. This would lead to huge algebraic linear systems to be solved at each time step, not affordable also for modern supercomputers, at least not for clinical applications going beyond prototypes and proofs of concept.

On the other hand, in many applications the level of information of 3D models exceeds the accuracy requested, in particular when we aim at modeling the dynamics occurring at the systemic more than at a local level. In this case it is preferable to adopt reduced models for which the computational efficiency and the systemic breath are considered more important than the local accuracy. One-dimensional (1D) models for the description of blood flow in a compliant vessel where the only space coordinate is the one associated with the vessel axis may provide a good trade-off among the different requirements. They have been introduced almost 250 years ago by L. Euler [60], and then rediscovered in the second half of the XX century in [14] - see also [105, 106]. The construction of these models is the result of two steps.

1. The description of motion of an incompressible fluid in a single compliant pipe. Only the axial dynamics is included; several simplifying assumptions are postulated - as we see later on - to apply conservation of mass and momentum along one space dimension. A suitable constitutive law is introduced to describe the relation between pressure and area of the pipe to include the arterial compliance;

2. The coupling of different segments composing the arterial tree by writing appropriate interface conditions between the single-segment models obtained at the previous step.

These reduced models do not allow to describe secondary flows. However, they provide average quantities at a very low computational time, a desirable feature that has been exploited since the '80s (see, e.g., [7, 115, 98]). It is worth reminding the book [159] reporting accurate investigations of the circulatory systems by means of Euler-like models.

Let us detail hereafter steps 1 and 2 . 


\subsubsection{The Euler model for an arterial segment}

One dimensional models may be derived in different ways. One of the most popular (and more sound from a physical standpoint) moves from the full 3D model and several simplifying assumptions on the behavior of the flow, the structure and their interaction. Hereby, we briefly sketch these assumptions and the consequent modeling procedure. Ample details can be found, e.g., in [174] and [160].

We assume the fluid domain to be represented by a cylindrical geometry or more generally by a truncated cone. We refer for notations to Figure 2, where a cylindrical coordinate system $(r, \varphi, z)$ is outlined. We make the following simplifying assumptions: (i) the axis of the cylinder is fixed; (ii) for any $z$, the cross section $\mathcal{S}(t, z)$ is a circle (i.e. no dependence on the circumferential coordinate $\varphi$ is assumed) with radius $R(t, z)$; (iii) the solution of both fluid and structure problems does not depend on $\varphi$; (iv) the pressure is constant over each section $\mathcal{S}(t, z)$; (v) the axial fluid velocity $u_{z}$ is dominant vs the other velocity components; (vi) only radial displacements are allowed, so that the structure deformation takes the form $\boldsymbol{\eta}=\eta \boldsymbol{e}_{r}$, where $\boldsymbol{e}_{r}$ is the unit vector in the radial direction; more precisely, we set $\eta(t, z)=R(t, z)-R_{0}(z)$ where $R_{0}(z)$ is the reference radius at the equilibrium; (vii) the viscous effects are modeled by a linear term proportional to the flow rate; (viii) the vessel structure is modeled as a membrane with constant thickness. As for assumption (vii), this is justified by the well known Poiseuille solution for a 3D Newtonian incompressible fluid in a circular cylinder, where the effects of viscosity are actually proportional to the flow rate.

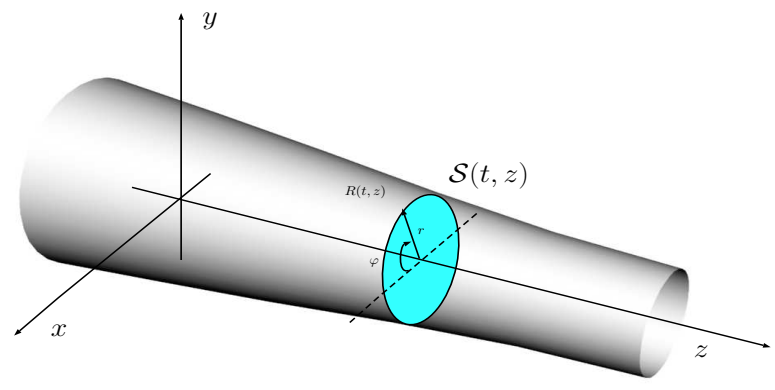

Figure 2: Fluid domain for the derivation of the 1D model.

To write the reduced model, we introduce the following quantities: $A(t, z)=|\mathcal{S}(t, z)|=$ $\pi R(t, z)^{2}$ (lumen section area), $\bar{u}(t, z)=A^{-1} \int_{\mathcal{S}(t, z)} u_{z}(t, z) d S$ (mean velocity), $s(r / R)$ is a velocity profile such that $u_{z}(t, r, z)=\bar{u}(t, z) s(r / R(t, z)), Q(t, z)=\rho_{f} \int_{\mathcal{S}(t, z)} u_{z} d S=\rho_{f} A(t, z) \bar{u}(t, z)$ (flow rate), $P(t, z)=A^{-1} \int_{\mathcal{S}(t, z)} p(t, z) d S$ (mean pressure).

As for the structure and its interaction with the fluid, we need a closure condition that states a functional dependence of the pressure on the lumen area (or equivalently on the displacement $\eta_{r}$ ) of the following form

$$
P(t, z)=P_{e x t}+\psi\left(A(t, z), A_{0}(z), \boldsymbol{\beta}(z)\right),
$$

where $\psi$ is a given function satisfying $\frac{\partial \psi}{\partial A}>0, \psi\left(A_{0}\right)=0$ and $P_{\text {ext }}$ the external pressure. Here $\boldsymbol{\beta}$ is a vector of parameters describing the mechanical properties of the membrane. The condition on $\frac{\partial \psi}{\partial A}$ responds to the intuitive expectation that the area gets larger when the pressure increases. 
By integrating over the sections $\mathcal{S}$ the momentum fluid equation (1a) in the $z$-direction and the mass conservation law (1b), we obtain the following system

$$
\frac{\partial \boldsymbol{U}}{\partial t}+\mathrm{H}(\boldsymbol{U}) \frac{\partial \boldsymbol{U}}{\partial z}+\boldsymbol{B}(\boldsymbol{U})=\mathbf{0} \quad z \in(0, L), t>0,
$$

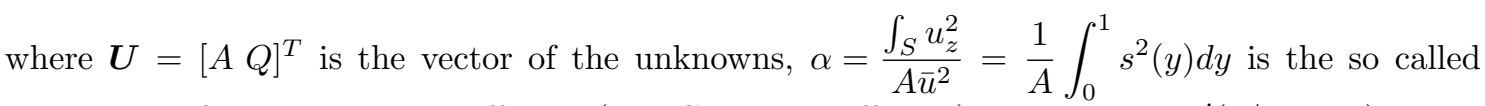
momentum flux correction coefficient (aka Coriolis coefficient), $K_{r}=-2 \pi \mu s^{\prime}(r / R=1)$ is the friction parameter (due to the viscous nature of the fluid), $c_{1}=\sqrt{\frac{A}{\rho_{f}} \frac{\partial \psi}{\partial A}}$, while

$$
\begin{array}{r}
\mathrm{H}(\boldsymbol{U})=\left[\begin{array}{ll}
0 & 1 \\
c_{1}^{2}-\alpha\left(\frac{Q}{A}\right)^{2} & 2 \alpha \frac{Q}{A}
\end{array}\right], \\
\boldsymbol{B}(\boldsymbol{U})=\left[\begin{array}{l}
0 \\
K_{r} \frac{Q}{A}+\frac{A}{\rho_{f}} \frac{\partial \psi}{\partial A_{0}} \frac{\partial A_{0}}{\partial z}+\frac{A}{\rho_{f}} \frac{\partial \psi}{\partial \boldsymbol{\beta}} \frac{\partial \boldsymbol{\beta}}{\partial z}
\end{array}\right]
\end{array}
$$

represent the flux matrix and the dissipation vector term. A complete derivation of the model can be found e.g. in [159, 105], and [160].

Alternatively, one could introduce the conservative form of the 1D system, which reads

$$
\frac{\partial \boldsymbol{U}}{\partial t}+\frac{\partial \boldsymbol{F}(\boldsymbol{U})}{\partial z}+\boldsymbol{S}(\boldsymbol{U})=\mathbf{0} \quad z \in(0, L), t>0,
$$

where $\boldsymbol{F}=\left[\begin{array}{ll}Q & \alpha Q^{2} / A+C_{1}\end{array}\right]^{T}$ and $\boldsymbol{S}=\boldsymbol{B}-\left[\begin{array}{ll}0 & \frac{\partial C_{1}}{\partial A_{0}} \frac{d A_{0}}{d z}+\frac{\partial C_{1}}{\partial \boldsymbol{\beta}} \frac{d \boldsymbol{\beta}}{d z}\end{array}\right]^{T}$, with $C_{1}=\int_{A_{0}}^{A} c_{1}^{2}$.

For blood flow a classical choice of the velocity profile is $s(y)=\gamma^{-1}(\gamma+2)\left(1-y^{\gamma}\right)$. For $\gamma=1$ we have $\alpha=1$ (flat profile), for $\gamma=2$ we have $\alpha=4 / 3$ (parabolic profile). Accordingly, we have $K_{r}=2 \pi \mu(\gamma+2)(=8 \pi \mu$ for a parabolic profile). Other, more sophisticated choices can be operated. For instance in [8] the pulsatile Womersley profile - that is, the unsteady periodic counterpart of the Poiseuille solution for the Navier-Stokes problem in a cylinder - is accounted for, while in [23] an approximated velocity profile is generated at each time step by solving simplified equations near the wall and in the core of the vessel.

The term $\frac{\partial A_{0}}{\partial z}$ in $\boldsymbol{B}$ is typically non-positive, as it accounts for the so-called vessel "tapering", i.e. the fact that the area of the lumen reduces when proceeding from proximal to distal direction. The term $\frac{\partial \boldsymbol{\beta}}{\partial z}$ originates from possibly different mechanical properties along the vessel, to describe, for example, the presence of plaques or vascular prostheses. A special treatment of these terms obtained by regarding $A_{0}$ and $\boldsymbol{\beta}$ as fictitious unknowns to be added to the system, is proposed in [142].

If $A>0$, system (7) has two distinct real eigenvalues (see, e.g., [174])

$$
\lambda_{1,2}=\alpha \bar{u} \pm \sqrt{c_{1}^{2}+\bar{u}^{2} \alpha(\alpha-1)},
$$

hence it is strictly hyperbolic (see e.g. [124]). Under physiological conditions, $c_{1}>>\alpha \bar{u}$, yielding $\lambda_{1}>0$ and $\lambda_{2}<0$, thus we have two waves traveling in opposite directions.

A simple membrane law (6) can be obtained by (5) by dropping the shear and inertial terms, leading to the following algebraic relation [67, 72],

$$
\psi\left(A, A_{0}, \beta\right)=\beta \frac{\sqrt{A}-\sqrt{A_{0}}}{A_{0}}, \quad \text { with } \quad \beta=\frac{\sqrt{\pi} H_{s} E}{1-\nu^{2}},
$$


where $\nu$ is the Poisson modulus of the membrane, $E$ its Young modulus, and $H_{s}$ its thickness, yielding $c_{1}=\sqrt{\frac{\beta \sqrt{A}}{2 \rho_{f} A_{0}}}$. This simple law, stating that the membrane radial displacement $\eta_{r}$ is linearly proportional to the fluid pressure, is successfully considered in many applications, see, e.g., $[197,133,89]$. Other laws have been proposed to account for other features of the arterial wall. For example the following law stems from the generalized string model [177, 210]

$$
\psi=m \frac{\partial^{2} A}{\partial t^{2}}-\gamma \frac{\partial A}{\partial t}-a \frac{\partial^{2}}{\partial z^{2}}\left(\sqrt{A}-\sqrt{A_{0}}\right)+\beta \frac{\sqrt{A}-\sqrt{A_{0}}}{A_{0}},
$$

where $m=\frac{\rho_{s} H_{s}}{2 \sqrt{\pi A_{0}}}$ is the mass of the membrane, $\gamma$ a coefficient accounting for visco-elastic effects and $a$ for the longitudinal pre-stress [78]. This generates three differential extra-terms in the momentum equation that account for the inertial, visco-elastic and pre-stressed effects, respectively (see [67] for the explicit expression of $\mathrm{H}$ and $\boldsymbol{B}$ in (7)). In [67] numerical results show that the wall-inertia term is important for large mass and/or high frequencies, the visco-elasticity term gives a small contribution, whereas the longitudinal pre-stress is important for strong area gradients (i.e. in presence of severe tapering or stenosis).

Different approaches have been introduced so far to account for visco-elastic effects. For example, in [7] the author considers a dynamic Young modulus which introduces a phase difference between applied forces and resulting displacements. Non-linear elastic effects are described in $[99,183]$, by splitting the membrane law in a non-linear elastic part and in a visco-elastic part. The first term is given by a relation like (11) where however the parameter $\beta$ depends non-linearly on the pressure. As for the visco-elastic term, the authors consider the convolution product between the elastic area and the derivative of a suitable creep function. The numerical results reported in $[192,183]$ show the importance of including non-linear terms and visco-elastic effects for the peripheral districts.

A more general membrane law is given by the following expression

$$
\psi=\beta\left(\left(\frac{A}{A_{0}}\right)^{n_{1}}-\left(\frac{A_{0}}{A}\right)^{n_{2}}\right),
$$

see [207]. For collapsible districts such as the veins, in [142] the authors propose to use $n_{1}=10$ and $n_{2}=3 / 2$, which allows to properly describe the high compliance of the veins. For a recent review on the $1 \mathrm{D}$ modelling of the venous system, see [206].

\subsubsection{Assembling a network of $1 D$ tracts}

Once a model to describe a single tract is available, we can connect many of such districts to represent a network of $1 \mathrm{D}$ models, in view of a description of the whole large size arterial (and, possibly, venous) system. One of key aspects to obtain realistic networks composed of several districts is modeling the bifurcations. As a matter of fact, at the continuous level, mass and momentum conservations hold at the bifurcations too. Referring to Figure 3 , let $\mathcal{B}^{1}$ be the proximal branch of the bifurcation and $\mathcal{B}^{2}, \mathcal{B}^{3}$ the distal branches. Then, by denoting $Q^{i}$ and $A^{i}(i=1,2,3)$ the flow rate and area of the branch $\mathcal{B}^{i}$ at the bifurcation point, the continuity conditions read

$$
\left\{\begin{array}{l}
Q^{1}=Q^{2}+Q^{3} \\
P_{t}^{1}=P_{t}^{2}=P_{t}^{3}
\end{array}\right.
$$


where $P_{t}^{i}=P_{\text {ext }}+\psi\left(A^{i}, A_{0}^{i}, \boldsymbol{\beta}^{i}\right)+\frac{\rho_{f}}{2}\left(\frac{Q^{i}}{A^{i}}\right)^{2}, i=1,2,3$, is the total pressure in the $i$-th branch. These conditions descend from the continuity of mass and momentum, respectively. With these interface conditions at the bifurcations, the $1 \mathrm{D}$ network undergoes a stability estimate that ensures energy conservation (up to the dissipative terms), see [2, 67, 194].

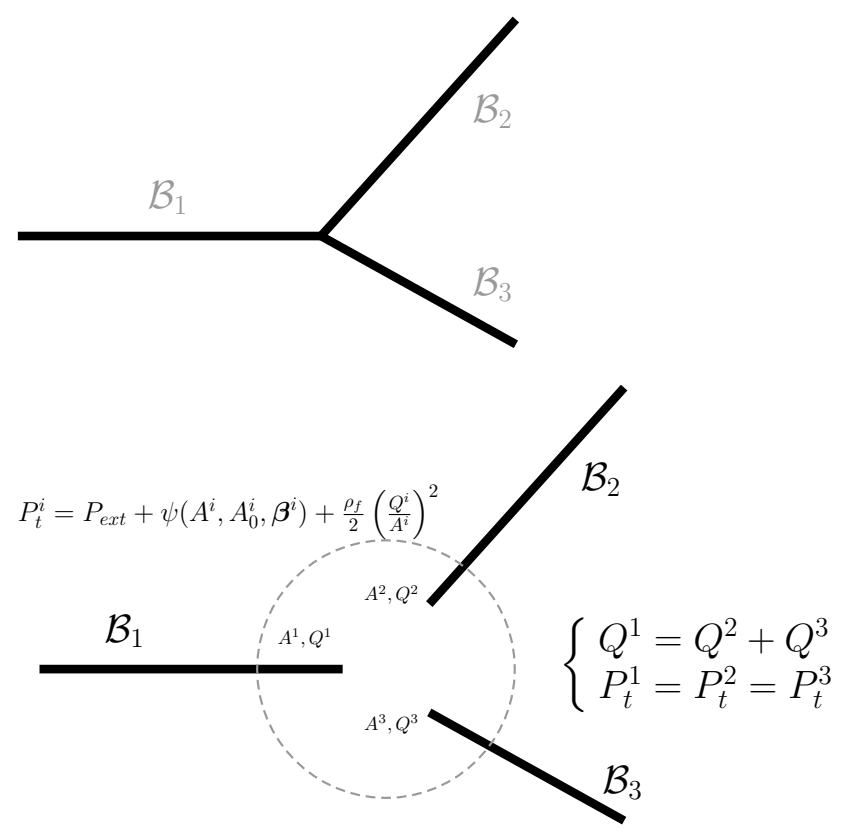

Figure 3: Scheme of a 1D vessel bifurcation.

\subsubsection{Numerical discretization}

For the numerical solution of problem (7), a common approach is based on the Taylor-Galerkin scheme, and more precisely the Lax-Wendroff scheme coupled with Finite Element space discretization, due to its excellent dispersion properties [65]. This scheme is explicit, so it is conditionally stable under the CFL condition

$$
\Delta t \leq \frac{1}{\sqrt{3}}\left[\frac{h}{\left(\sqrt{c_{1}^{2}+\bar{u}^{2} \alpha(\alpha-1)}+|\bar{u}|\right)}\right],
$$

where $h$ is the spatial gridsize and $\Delta t$ the time step, that for simplicity we have assumed to be constant.

This method may be used in association with an operator splitting technique [67, 131], where the flow rate is split into two components, one satisfying the pure elastic problem and the second one the visco-elastic correction.

A high-order discontinuous Galerkin approximation is considered in [194, 193], allowing to propagate waves of different frequencies without suffering from excessive dispersion and diffusion 
errors, so to reliably capture the reflection at the junctions induced by tapering. Alternatively, a high-order finite volume scheme is presented in [143] and a space-time finite element method is proposed in [221]. Recently, a series of benchmark test cases with an increasing degree of complexity is presented in [38] to compare different numerical schemes.

\subsubsection{Further developments and comments}

Validation of $1 D$ models.. The accuracy of the solution provided by the 1D model is addressed in several works. Among them, we cite [7], where a network of 128 vessels is considered for the description of the whole system, and the numerical results have been compared successfully with measurements taken in the ascending aorta, descending aorta, brachiocephalic and right common iliac arteries; [197], where the numerical results obtained in aorta are shown to be in good agreement with MRI measurements; [133, 142], where a comparison with in vitro measurements is performed for a complete network of the system; [183], where a comparison with clinical measurements is addressed, with a particular focus on the circle of Willis; [197], where a validation is presented for the case of a by-pass graft.

In order to remove the quite stringent assumption of rectilinear vessels, a $1 \mathrm{D}$ modeling procedure on general axes is investigated in [87]. A follow up of this seminal paper can be found in [2].

Tuning the parameters.. The choice of suitable parameters in the 1D system, in particular in the membrane law, is crucial to obtain accurate solutions. Besides parameters settings based on a "trial and error" approach, a more sophisticated strategy based on the minimization of a suitable functional is proposed in [132] and then analyzed and applied to a real case in [135]. An alternative approach based on the so called director theory can be found in [184].

A RELATED TOPIC CONCERNS THE INCLUSION OF UNCERTAINTY IN THE PARAMETERS. IN [224], THE PARAMETERS APPEARING IN THE 1D MODEL ARE MODELLED AS RANDOM VARIABLES, LEADING TO STOCHASTIC EQUATIONS FOR THE ARTERIAL NETWORK.

Accounting for the surrounding tissue.. The presence of surrounding tissues can be integrated in 1D models in the description of the vascular membrane. For example, if the surrounding tissue is supposed to behave as an elastic body, we deduce from (3) that the effective elastic modulus $\widehat{\beta}$ to be used in the vessel law is $\widehat{\beta}=\beta+\alpha_{S T}$ [72].

Hierarchically refined $1 D$ models.. One of the possible drawback of $1 \mathrm{D}$ models presented so far is that the dynamics occurring transversally to the axis of the domain is neglected. Even though over a systemic scale this may be acceptable, local dynamics may be important and worth to be included in the model. As an alternative to full 3D modeling (and somehow to the geometric multiscale models addressed later on), in [165] a form of hierarchical modeling is introduced to reduce the full 3D problem to a system of "psychologically" 1D models. Conceptually, this approach consists of coupling a classical finite element discretization along the axial direction with a spectral approximation of the transversal components. The rationale is that a few modes are expected to be enough for reliably capturing the transverse dynamics. In addition, the number of modes may be adaptively selected in different regions of the system $[166,1]$. See [164] for a comprehensive introduction to this method and [25] for applications to hemodynamics. 


\subsection{Lumped Parameter Models}

In early days, modeling of large portions of the circulation was almost invariably based on the concept of compartment. A compartment is a functional unit that makes sense to consider as homogeneous. Per se, this is a quite generic definition, since "being homogeneous" depends on the application and on the purpose of the models. We may say that for modeling circulation, a compartment is a set of vascular districts that is appropriate to regard as a unit for the application at hand.

For instance, when investigating fluid-dynamics in the aortic arch, local details of blood flow in the lower limbs are most likely not needed, yet it is important to include the macroscopic effects induced by peripheral sites on the region of interest. This is even more important in case of pathologies. This may also result in simple "in-out" relations or transfer functions. The latter do not necessarily rely upon physically based arguments and sometimes empirical models with an accurate parameter identification can work.

In this work we are interested in performing a dimensionally heterogeneous coupling, where compartment models are eventually coupled with the physically based 3D and 1D descriptions of the previous two sections. For this reason, here we proceed with a bottom-up approach, where we first motivate possible lumped parameter descriptions of basic functional units like an arterial segment sticking with a physical modeling and following up the arguments of the previous section. Moving from these elementary components, we then consider more complex functional units and show how they can be assembled from elementary components.

\subsubsection{Lumped Parameter Modeling of an Arterial Tract}

Let us consider an arterial segment as done in Figure 2. We are not concerned about the actual shape of the segment and its axis, we simply define the abscissas $z_{p}$ and $z_{d}$ corresponding to the proximal and distal boundaries of the segments respectively. Still using the notation introduced in Section 2.2.1, our aim is to describe the average flow rate and pressure in this region, defined respectively as

$$
\widehat{Q}(t)=\frac{1}{l} \int_{z_{p}}^{z_{d}} Q(t, z) d z=\frac{\rho_{f}}{l} \int_{z_{p}}^{z_{d}} \int_{\mathcal{S}(t, z)} u_{z}(t, z) d S d z, \quad \widehat{P}(t)=\frac{1}{l} \int_{z_{p}}^{z_{d}} P(t, z) d z=\frac{1}{V} \int_{z_{p}}^{z_{d}} \int_{\mathcal{S}(t, z)} p(t, z) d S d z,
$$

where $l$ is the length of the segment and $V$ the volume.

Our aim is to describe the dynamics in time of $\widehat{Q}$ and $\widehat{P}$ in the compartment. Moving from the Euler equations (7) we integrate along the axial direction. In doing this, we add some new simplifications. We drop the nonlinear convective term as in the peripheral sites the Reynolds number is usually fairly small, hence the impact of the nonlinear term on the compartment dynamics is secondary. In addition, we consider the area and the other parameters to be constant in the region where we perform the average. This basically means that we will retain nominal values for these parameters, as representative of the entire compartment.

If we take the longitudinal average of the tract on the momentum equation, we loose any space dependence and obtain the ordinary differential equation

$$
\frac{\rho_{f} l}{A_{0}} \frac{d \widehat{Q}}{d t}+\frac{\rho_{f} K_{R} l}{A_{0}^{2}} \widehat{Q}+\widehat{P}_{d}-\widehat{P}_{p}=0
$$




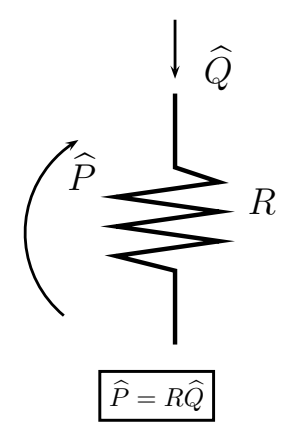

Resistance $\leftrightarrow$ Viscosity

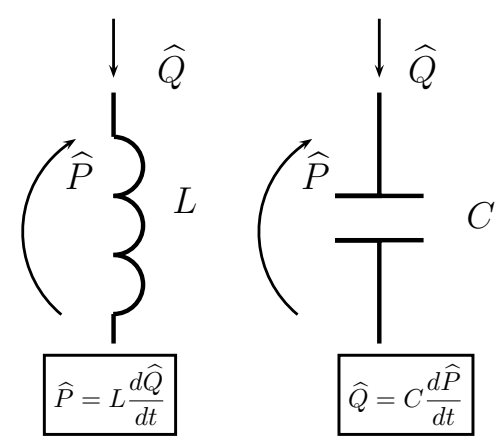

Inductance $\leftrightarrow$ Inertia
Capacitance $\leftrightarrow$ Compliance

Figure 4: Electric/Hydraulic analogy: the elementary components. In the box we report the mathematical description of each component.

where $\widehat{P}_{d}$ and $\widehat{P}_{p}$ are the distal and proximal pressure, respectively. When taking the longitudinal average of the mass conservation law, under the assumption that the time variations of pressure are linearly proportional to the time variation of the area, we obtain [160]

$$
\frac{\sqrt{A_{0}} l}{\beta} \frac{d P}{d t}+\widehat{Q}_{d}-\widehat{Q}_{p}=0
$$

where $\widehat{Q}_{d}$ and $\widehat{Q}_{p}$ are the distal and proximal flow rate, respectively.

The two equations (15)-(16) represent a compartment model for an arterial tract. Notice how three main effects are driving the motion of blood, (i) the blood inertia, (ii) the interaction with the wall, and (iii) the viscous resistance. While these effects are distributed along the $1 \mathrm{D}$ domain in the Euler equations (7), they are lumped in specific terms of the equations (15)-(16). In fact, the term $L \frac{d \widehat{Q}}{d t}$, with $L=\frac{\rho_{f} l}{A_{0}}$, corresponds to the blood acceleration, so it is an inertial term. The algebraic term $R \widehat{Q}$, with $R=\frac{\rho_{f} K_{R} l}{A_{0}^{2}}$, stems from the blood viscosity, while $C \frac{d \widehat{P}}{d t}$, with $C=\frac{\sqrt{A_{0}} l}{\beta}$, is due to the time variation of the section as a consequence of fluid-structure interaction.

Systems formally similar to (15)-(16) occur in different fields of applied mathematics. For instance they are obtained when studying the equations of a hydraulic network [108] (with a coupling of 1D-0D models) and of a co-axial cable (see e.g. [188, 169]). In this respect, it is possible (and popular) to establish an analogy between terms in the electrical as well as in the fluid-dynamics contexts, where the role of the flow rate for fluid-dynamics is played by the current, and the pressure is corresponded by the voltage. This allows to adopt the symbolism of electrical circuits also in modeling the circulation. In particular, the three contributions mentioned above are mathematically described by simple algebraic and differential equations, stated in Table 1 . The corresponding symbols in Circuits Theory are depicted in Fig. 4.

The parameters involved in these equations depend on the specific features of the arterial tract. For instance, if we assume a circular cylinder with radius $R_{0}$ and a Poiseuille like flow, we obtain 


\begin{tabular}{|c|c|c|c|}
\hline Fluid-dynamics & Electric & Parameter & Equation \\
\hline \hline Viscosity & Resistance & $R$ & $\widehat{P}=R \widehat{Q}$ \\
\hline Inertia & Inertance & $L$ & $\widehat{P}=L \frac{d \widehat{Q}}{d t}$ \\
\hline Compliance & Conductance & $C$ & $\widehat{Q}=C \frac{d \widehat{P}}{d t}$ \\
\hline
\end{tabular}

Table 1: Table of analogy for hydraulic networks-electrical circuits.

the following parameters [160],

$$
R=\frac{8 \mu l}{\pi r_{0}^{4}}, \quad L=\frac{\rho_{f} l}{\pi R_{0}^{2}}, \quad C=\frac{3 \pi R_{0}^{3} l}{2 E H_{s}} .
$$

In general, the fine tuning of these parameters may be troublesome in particular in a patientspecific setting. Beyond specific assumptions on the shape and the flow regime in the artery, this parameter estimation may be pursued also by experimental or data assimilation procedures [22].

To finalize the circuit analogy, we may conceptually organize the different lumped contributions to the dynamics into different sequences from the proximal to the distal side of the artery. For instance, we may consider to localize the unknown pressure $\widehat{P}$ at the proximal section, so to have $\widehat{P} \approx \widehat{P}_{p}$ and to assume correspondingly that the distal pressure $\widehat{P}_{d}$ is given. Similarly we assume that the flow rate $\widehat{Q}$ is approximated by $\widehat{Q}_{d}$ and that the proximal flow rate $\widehat{Q}_{p}$ is prescribed. Then, from (15)-(16), the Lumped Parameter Model (LPM) reads

$$
\begin{aligned}
& \widehat{P}-L \frac{d \widehat{Q}}{d t}-R \widehat{Q}=\widehat{P}_{d}, \\
& C \frac{d \widehat{P}}{d t}+\widehat{Q}=\widehat{Q}_{p},
\end{aligned}
$$

corresponding to the circuit depicted in Fig. 5(a). Other sequences are depicted in the other panels of the same figure. Notice that the difference among all these schemes is drawn by different choices to approximate the unknowns $\widehat{P}$ and $\widehat{Q}$. For example, in Fig. 5(b) we have used $\widehat{P} \approx \widehat{P}_{d}$ and $\widehat{Q} \approx \widehat{Q}_{p}$, and so on. With a slight abuse of notations, we may say that the different schemes differ because of the different "boundary conditions" (the misuse being motivated by the fact that we have actually lost space dependence in these models) - see [160] for a more extensive discussion. We can consider likewise different ways for localizing the different effects (that are actually distributed in space) leading to different schemes. Even though these schemes are equivalent in terms of functionality, their different formulation plays an important role when coupling with dimensionally heterogeneous models, as we will see in Section 5 .

A central tool for the quantitative study of linear circuits and then of linearized hydraulic networks is the Fourier transform, that allows to promptly find the frequency response of the system by downscaling the differential equations in time to algebraic equations in the frequency 


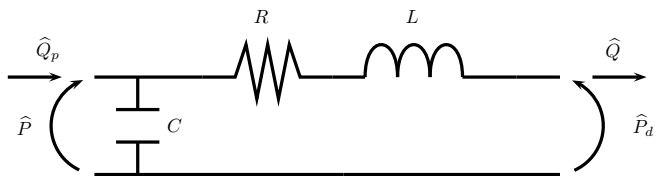

a)

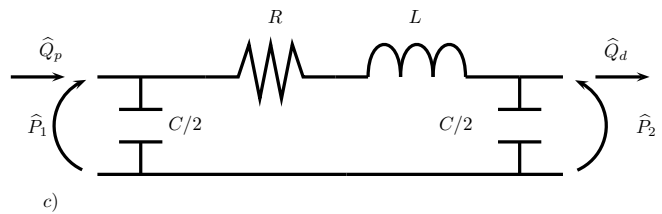

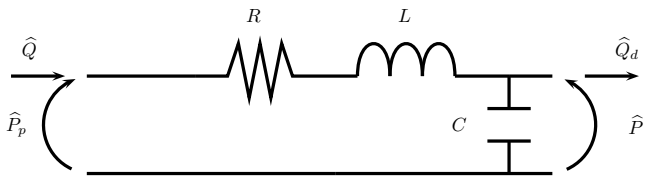

b)

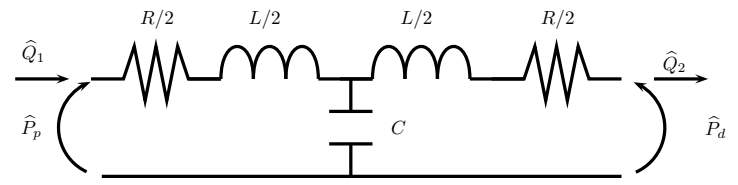

d)

Figure 5: Different lumped parameter schemes for an arterial tract. (a) $\mathcal{L}$ network. (b) $\mathcal{L}$-inverted network. (c) $\pi$ network. (d) $\mathcal{T}$ network.

domain [186]. More precisely, let us denote by $\mathcal{R}(\omega)$ the Fourier transform of a function $R(t)$,

$$
\mathcal{R}(\omega)=\int_{-\infty}^{\infty} e^{-j \omega t} R(t) d t
$$

where $\omega$ is the frequency and $j$ is the imaginary unit. Then, thanks to the properties of Fourier transform, we promptly find that for the LPM (17)

$$
\left[\begin{array}{l}
\widehat{\mathcal{P}}(\omega) \\
\widehat{\mathcal{Q}}(\omega)
\end{array}\right]=\mathrm{M}(\omega)\left[\begin{array}{l}
\widehat{\mathcal{P}}_{d}(\omega) \\
\widehat{\mathcal{Q}}_{p}(\omega)
\end{array}\right], \quad \text { with } \quad \mathrm{M}(\omega)=\left[\begin{array}{cc}
1-\omega^{2} L C+j \omega R C & R+j \omega L \\
j \omega C & 1
\end{array}\right]
$$

where the matrix $\mathrm{M}(\omega)$ is called transfer matrix. The time dependent functions $\widehat{P}(t)$ and $\widehat{Q}(t)$ can be computed by inverting the transform (antitransform).

\subsubsection{From an arterial tract to a compartment}

The lumped parameter modeling of an arterial tract was based on averaging axially the Euler equations with the addition of some simplifying assumptions, so it was essentially a physically based description. When modeling more complex vascular districts or regions, we may either adopt empirical arguments aimed at a pure functional description, or patching together several elementary tracts. Hereafter, we describe the two approaches, the former in particular is used for simple descriptions of terminal vessels as the ones depicted in Fig. 6. In this case, the concept of peripheral impedance, i.e. the transfer function between the Fourier transform of the peripheral flow rate and pressure, is of primary relevance.

The latter approach is based on classical continuity arguments and may be used for an accurate description of several segments of the circulatory network. Among the compartments that it is worth considering as a unit when described by a LPM, we include the heart too.

Lumped Parameter Models of terminal vessels. In many applications one simple compartment is enough to describe the entire arterial system downstream a region of interest, especially if 
we are interested just in the effects of peripheral circulation on that region. We remind here just the most popular LPMs used to this aim.

(a) In the windkessel model [223], the action of the peripheral districts is modeled by an average resistance and capacitance, as depicted in Fig. 6(a). The name is windkessel, after Otto Frank [77], as the device to convert periodic to continuous water flow by German firemen. The impedance $Z(\omega)$ at the entrance of the windkessel model is the transfer function between the Fourier transform of the flow rate and of the pressure, $\widehat{\mathcal{P}}(\omega)=Z_{w k}(\omega) \widehat{\mathcal{Q}}(\omega)$ and it reads

$$
Z_{w k}(\omega)=\frac{R}{1+j R C \omega} .
$$

(b) In order to have a better fitting with data, this original model has been modified in the so called 3-element windkessel (sometimes called Westkessel to honor N. Westerhof). In this model, a second resistance is added before the windkessel compartment, having the terminal impedance

$$
Z_{w k 3}(\omega)=\frac{R_{1}+R_{2}+j \omega R_{1} R_{2} C}{1+j \omega R_{2} C},
$$

see Fig. 6(b).

(c) A further improvement foresees the addition of an inductance element as illustrated in Fig. 6(c) (called 4-element windkessel model $[198,199])$. The impedance in this case reads

$$
Z_{w k 4}(\omega)=\frac{R_{1} R_{2}-C R_{1} R_{2} L \omega^{2}+j \omega\left(R_{1}+R_{2}\right)}{R_{1}-C R_{2} L \omega^{2}+j \omega\left(L+C R_{1} R_{2}\right)} .
$$

To complete this description we mention also the low pass filter proposed in [90] (corresponding to the 3-elements windkessel model with $R_{1}=0$ ) as a scheme for the distal circulation of cerebral vasculature.

The family of these "windkessel like" models serves the purpose of describing peripheral districts at a first level of approximation. Unfortunately, it may fail for a more precise description of propagative dynamics associated with the peripheral circulation. For this reason, more sophisticated approaches have been proposed. In particular, the structured tree model [153] assumes an asymmetric self-similar structure for the peripheral network, where each segment is represented by a transfer function retrieved from the (linear) wave theory and an appropriate constitutive law is assumed for branching. Below a certain threshold of the vessel radius a purely resistive impedance is assumed to hold. See [152] for more details, while recently an alternative formulation has been proposed in [48]. We also mention [111] and [205] where proper outflow conditions for the coronary tree and cerebral aneurysms, respectively, are discussed.

Connecting different tracts. Another way for describing the arterial network is based on the connection of several elementary tracts like the ones introduced in the previous section. To do this, we need appropriate matching conditions at the interface of the different units. These stem from continuity arguments derived from mass and momentum conservation, similarly to what is for connecting different tracts in 1D models as in Sect. 2.2. However, in this case, since the nonlinear convective term is dropped, the total pressure and the pressure coincide. In the analogy between hydraulic and electric networks, these principles are corresponded by the so called Kirchhoff laws, that formulate conservation of mass at nodes, while at nets ensure momentum conservation. 

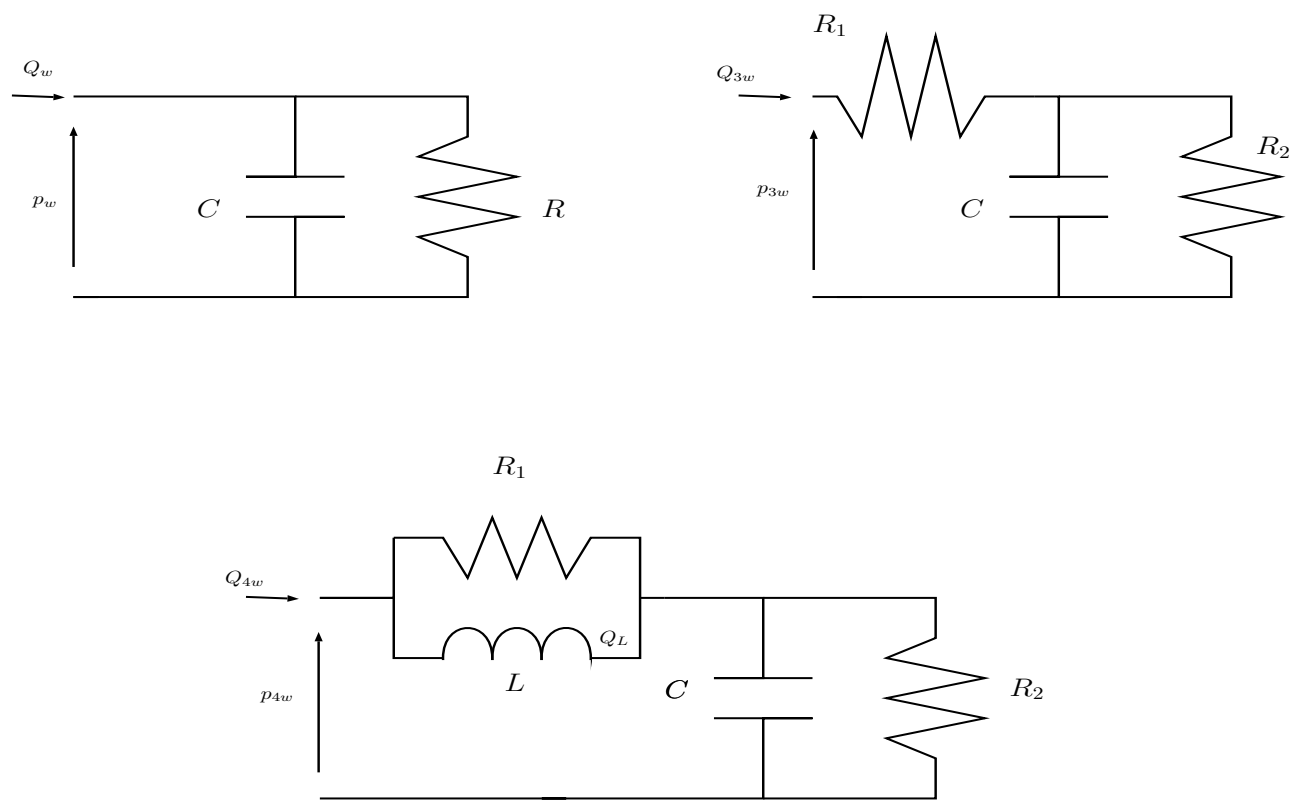

Figure 6: Lumped parameter models of terminal vessels. (a) windkessel; (b) 3-element windkessel; (c) 4-element windkessel.

In the frequency domain, continuity of flow rate and pressure yields a convenient description of the transfer function of the network. If a sequence of two compartments $\mathcal{C}_{1}$ and $\mathcal{C}_{2}$ is described by the transfer matrices $\mathrm{M}_{1}$ and $\mathrm{M}_{2}$, the interface conditions read

$$
\widehat{P}_{d, 1}(t)=\widehat{P}_{p, 2}(t), \quad \widehat{Q}_{d, 1}(t)=\widehat{Q}_{p, 2}(t),
$$

so that in the frequency domain we have

$$
\left[\begin{array}{l}
\widehat{\mathcal{P}}_{p, 1} \\
\widehat{\mathcal{Q}}_{p, 1}
\end{array}\right]=M_{1}\left[\begin{array}{l}
\widehat{\mathcal{P}}_{d, 1} \\
\widehat{\mathcal{Q}}_{d, 1}
\end{array}\right], \quad\left[\begin{array}{l}
\widehat{\mathcal{P}}_{p, 2} \\
\widehat{\mathcal{Q}}_{p, 2}
\end{array}\right]=M_{2}\left[\begin{array}{l}
\widehat{\mathcal{P}}_{d, 2} \\
\widehat{\mathcal{Q}}_{d, 2}
\end{array}\right] \Rightarrow\left[\begin{array}{l}
\widehat{\mathcal{P}}_{p, 1} \\
\widehat{\mathcal{Q}}_{p, 1}
\end{array}\right]=M_{1} M_{2}\left[\begin{array}{l}
\widehat{\mathcal{P}}_{d, 2} \\
\widehat{\mathcal{Q}}_{d, 2}
\end{array}\right],
$$

showing that the transfer matrix of the sequence is simply given by the product of the matrices of the components.

In Fig. 7 we represent the sequence of three compartments, the first two are two arterial tracts, followed by a 3-element windkessel.

Lumped Parameter Models of the heart. The heart is a complex organ featuring different dynamics (electrical, fluid and structure) ranging over multiple scales, from the cell to the organ one. However, when simulating the entire circulation in most of the cases we are not interested in all these different aspects and the heart can be considered just as a functional unit triggering the dynamics of the entire system. The coupling between the heart and the circulation is a truly two-way dynamics [145] and the influence of the arterial network on the heart functionality may be important in particular in presence of pathologies that induce a cardiac overload. In clinical 


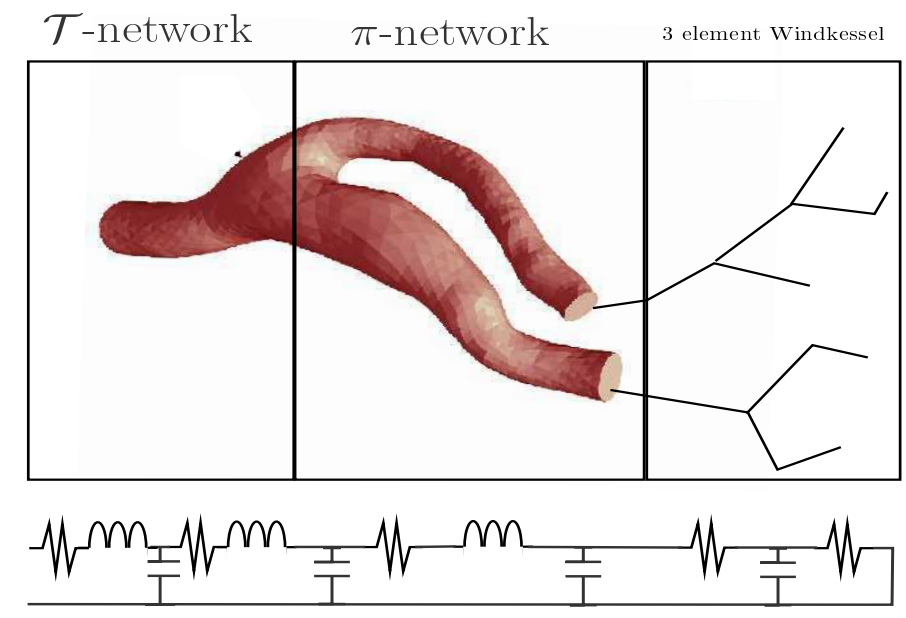

Figure 7: Connection of different compartments for a carotid bifurcation. The distal district is given by a 3-element windkessel.

practice a lumped parameter model of this coupling may help clarifying these mutual interactions [68].

An empirical and simple description of the heart leading to lumped parameter models is given for instance in [102] where each ventricle is represented as a deformable chamber whose compliance is time-dependent. In fact, let $E$ be the Young modulus of the cardiac tissue. Since muscles exert different actions during the heart beat, $E$ will be in general a function of time. Associated with this we introduce the compliance

$$
C(t)=\frac{2 \pi l_{0}^{3}}{E(t) h_{0}},
$$

where $l_{0}$ is a reference length for the ventricle and $h_{0}$ is a reference thickness of the myocardium. The classical Laplace law between pressure and volume of a pressurized chamber leads to the equation

$$
\widehat{Q}(t)=\frac{d C}{d t} \widehat{P}(t)+C \frac{d \widehat{P}}{d t}+M_{Q}(t),
$$

where $M_{Q}$ is the time dependent action of muscles. More complex models can be considered as well, see e.g. [187]. Coupling with the circulation is mediated by valves, whose behavior cannot be described by the elementary components depicted in Fig. 4. In the analogy with electric circuits, valves can be functionally represented by diodes. THE PRESENCE OF VALVES/DIODES INTRODUCE NONLINEAR TERMS IN THE ORDINARY DIFFERENTIAL SYSTEM ASSOCIATED WITH THE 0D model. An ideal Valve has a digital on-OFF Behavior (Flow Rate is positive WHEN THE PRESSURE DROP IS ZERO AND ZERO WHEN THE PRESSURE DROP IS NEGATIVE). IN PRACTICE, FROM THE NUMERICAL POINT OF VIEW, A MORE REALISTIC LAW IS SELECTED, WITH 


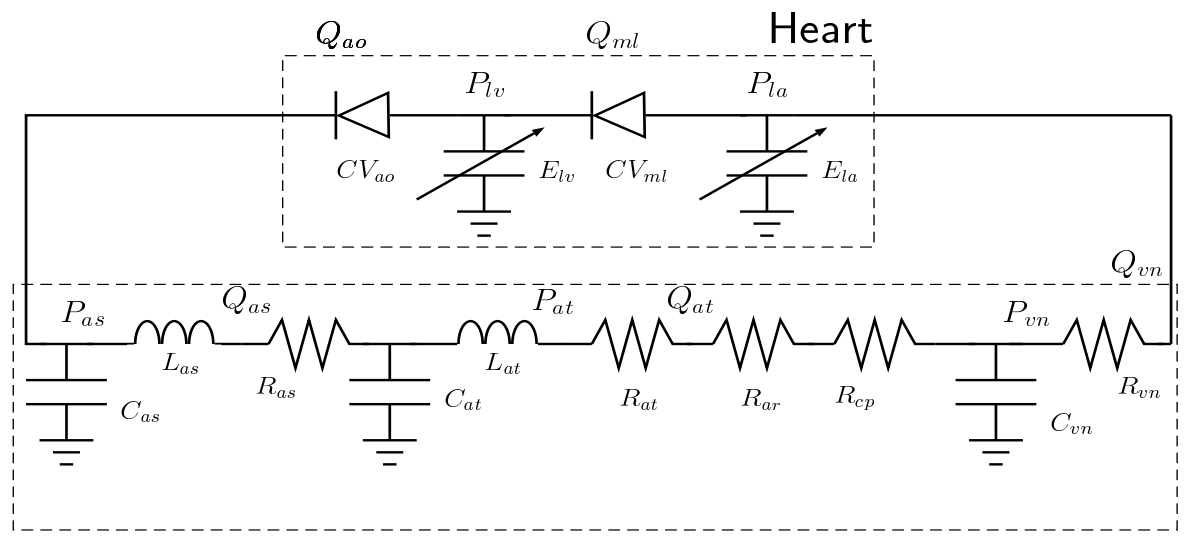

Systemic Loop

Figure 8: Schematic representation of the circulation by a lumped parameter model including the heart, the arterial system and the venous one. Adapted from models.cellml.org. $l v=$ left ventricle, $a o=$ aortic, $m l=$ mitral, $l a=l e f t$ atrium, $a s=$ aortic sinus, $a t=$ arteries, $a r=$ arterioles, $c p=$ capillaries, $v n=$ venous.

AN EMPIRICAL EXPONENTIAL RELATION BETWEEN PRESSURE AND FLOW RATE, MIMICKING THE ON-OFF SWITCH. THIS IS EVENTUALLY APPROXIMATED BY CLASSICAL METHODS FOR NONLINEAR EQUATIONS OR BY SEMI-IMPLICIT TIME ADVANCING SCHEMES (SEE, E.G., [175]).

Other MODEls HAVE BEEN INTRODUCED TO ACCOUNT, E.G., FOR DISEASED VAlVES [114] OR ATRIA CHAMBERS [125, 28].

An example of a complete network including the two ventricles, the arterial and the venous loop is reported in Fig. 8 .

\subsubsection{On the numerical solution of Lumped Parameter Models}

Linear networks can be solved analytically in the frequency domain, by standard application of transform techniques. However, in many applications of interest, the networks feature complex topologies and nonlinear elements like diodes, so numerical approximation in the time domain provides a viable tool for quantitative analysis. In mathematical terms, the combination of constitutive laws describing each compartment and the continuity interface condition between the compartments leads to the formulation of a system of differential and algebraic equations (DAE systems) that takes the form

$$
\begin{aligned}
& \mathrm{A} \frac{d \boldsymbol{y}}{d t}+\boldsymbol{f}(t, \boldsymbol{y}, \boldsymbol{z})=\mathrm{g}(t), \\
& \mathrm{G} \boldsymbol{y}+\mathrm{M} \boldsymbol{z}=\boldsymbol{c}(t)
\end{aligned}
$$

$t>0$. Here $\boldsymbol{y}$ is the set of $n$ variables whose time dynamics describes the state of the system (pressure and flow rates in the different locations of the network associated with capacitance and inductance effects respectively) and $\boldsymbol{z}$ is a vector of $m$ additional variables needed to close the description of the network by the application of the balance conditions (Kirchhoff laws, given by $(18 \mathrm{~b})$ ); $\mathrm{A}$ is a $n \times n$ matrix, $\mathrm{G}$ is $m \times n$ and $\mathrm{M}$ is $m \times m$. The term $\boldsymbol{f}(t, \boldsymbol{y}, \boldsymbol{z})$ is in general nonlinear for the presence of diodes. $\boldsymbol{g}$ and $\boldsymbol{c}$ represent forcing terms. We assume that initial conditions 
$\boldsymbol{y}(0)=\boldsymbol{y}_{0}$ are prescribed at $t=0$. In the case of interest for cardiovascular applications, the square matrix $\mathrm{M}$ is generally nonsingular. This qualifies system (18) as an index 1 DAE system and we may reduce it to a classical Cauchy problem

$$
\begin{aligned}
& \mathrm{A} \frac{d \boldsymbol{y}}{d t}+\boldsymbol{f}\left(t, \boldsymbol{y}, \mathrm{M}^{-1}(\boldsymbol{c}-\mathrm{G} \boldsymbol{y})\right)=\mathrm{g}(t), \\
& \boldsymbol{y}(0)=\boldsymbol{y}_{0},
\end{aligned}
$$

for which several well established numerical methods exist [40]. Among the others we mention Runge Kutta methods that are particularly prone to time-adaptive strategies that allow an automatic selection of the time discretization step to fulfill user-defined accuracy requirements. We do not enter into details here, the interested reader is referred to $[120,176]$.

\subsubsection{Further developments}

The actual functioning of the circulatory system includes effects that cannot be simply described by fluid-dynamics and fluid-structure interaction equations. Living systems adapt to different conditions. For instance, running requires an increment in oxygen delivery to lower limbs that may be obtained by an increment of the blood pumping rate and a vasodilation in the districts interested by the physical exercise. These regulatory dynamics involve the interaction between fluid, mechanics and chemical reactions occurring in several parts of the network according to different mechanisms. For instance, a constant oxygen delivery to the brain tissues is guaranteed by complex dynamics that go under the name of autoregulation.

A comprehensive description of these aspects is out of the scope of the present work. We remind however that lumped parameter models are particularly fitted to provide quantitative models since they are able of covering large portions of the network and of providing simple but reliable empirical models of complex dynamics occurring at different time scales. The interested reader is referred to $[154,160]$ and the references therein mentioned.

FINALLY, WE MENTION THE EMERGING ISSUE OF INCLUDING UNCERTAINTY IN THE PARAMETERS OF THE 0D MODELS. THIS TOPIC HAS BEEN RECENTLY ADDRESSED IN [191] WHERE THE AUTHORS PROPOSE A FULLY BAYESIAN, MULTI-LEVEL APPROACH FOR LUMPED PARAMETER MODELS FOR TERMINAL VESSELS.

\section{Boundary conditions: what we have, what is missing}

When solving blood flow problems with any of the stand-alone models introduced in the previous section, we are faced with the issue of the data to be prescribed as boundary conditions for the 3D and $1 \mathrm{D}$ models or as forcing terms (that surrogate boundary conditions) for $0 \mathrm{D}$ models.

In clinical settings, data are retrieved from in vivo measures. This can be done in many different ways and an extensive analysis of possible sources of data is certainly out of scope of the present work. In general, measures may refer to pointwise velocities on a (strict) subset of inflow/outflow boundaries of a vascular district (e.g. with PC-MRI) or indirectly to average quantities like the flow rate over a section; for the pressure, data (in particular obtained by noninvasive measures) are almost invariably an average information over a section of interest. Quite often there are practical difficulties in retrieving data at the distal (outlet) boundaries and no patient-specific information is available. These practical problems clearly add specific issues to the boundary treatment of mathematical and numerical models. 
Specifically, if we are using a 3D model in deformable domains that correspond to tracts of arteries that are artificially truncated, the correct prescription of boundary conditions needs to address several concerns. On the one hand, we need to prescribe conditions consistent with the wave propagation dynamics described by the FSI that in particular avoid spurious reflections at the outlets. On the other hand, partial (or absent) measures need to be properly completed to make the 3D model well posed. The additional - somehow arbitrary - conditions need to be consistent with the physical problem. This is discussed in Sect. 3.1.

When dealing with 1D models, the lack of boundary data is in general less troublesome (as a matter of fact, one average-in-space quantity per boundary point makes the continuous problem well-posed). However, the hyperbolic nature of the 1D model still raises issues of prescribing conditions at the outlets in a way consistent with the wave propagation dynamics. In addition, numerical discretization usually requires extra conditions that are absent in the continuous problem, yet need to be retrieved in a way consistent with the orignal model. This calls for special care for boundary treatment as we will see in Sect. 3.2.

Finally, we have already pointed out that in spite of the lack of spatial dependence in $0 \mathrm{D}$ models, prescription of input physically corresponds to localized data and the type of those data needs to be compatible with the nature of the lumped parameter model. This is discussed in Sect. 3.3 .

\section{1. $3 D$ defective boundary problems}

Mathematical theory of the incompressible Navier-Stokes equations states that we need to prescribe three scalar conditions at each point of the boundary. This is almost invariably impossible in clinical settings. As pointed out, phase-contrast MRI provides for instance velocity data only in selected points of a vascular domain that typically do not cover the entire entrance section $\Gamma$ of a vascular district [141]. Alternatively, the flow rate $Q(t)$ can be obtained at $\Gamma$ by proper elaboration of data retrieved e.g. by the Echo-Doppler technique based on ultrasound [190] or by thermal images [129] - see also [226, 110]. Moreover, as we will see in Sect. 4 and 5, the prescription of defective data for the 3D model is a crucial issue in view of the geometric multiscale approach we are here reviewing. For all these reasons, we consider the following flow rate condition

$$
\rho_{f} \int_{\Gamma} \boldsymbol{u} \cdot \boldsymbol{n} d \gamma=Q
$$

Similarly, at both the inlet and outlet sections available pressure data $P(t)$ are considered representative of an average estimate, i.e. we have

$$
\frac{1}{|\Gamma|} \int_{\Gamma} p d \gamma=P .
$$

Conditions (19) and (20) are called defective in the sense that they prescribe just one scalar function over the entire section $\Gamma$, marking a clear gap between theory and practice that needs to be filled up to get quantitative solutions to the problem. Hereafter we illustrate some of the most common strategies to pursue this goal. 


\subsubsection{Flow rate condition}

Empirical approach.. The most immediate way to prescribe condition (19) consists in choosing a velocity profile $\boldsymbol{g}$ such that for each $t$

$$
\rho_{f} \int_{\Gamma} \boldsymbol{g}(t) \cdot \boldsymbol{n} d \gamma=Q(t) .
$$

In this way, flow rate data are converted into standard Dirichlet conditions. This is a popular strategy in computational hemodynamics. Classical choices for the velocity profile are the parabolic one, which works very well for example for flow simulations in the carotids [43], the flat one, which is quite often used for the ascending aorta [140], and the one based on the Womersley solution. Notice that both the parabolic and Womersley profiles require a circular section to be prescribed on. Non-circular sections require an appropriate morphing [94].

Unfortunately, in spite of its straightforward implementation, the a priori and arbitrary assumption on the velocity profile has a major impact on the solution, in particular in the neighborhood of the section. To reduce the sensitivity of the results to the arbitrary choice of the profile, the computational domain can be artificially elongated by the so called flow extensions, thus involving additional computational burden.

Augmented formulation based on Lagrange multipliers: rigid walls.. The augmented formulation was proposed in [66] for the case of rigid walls and for a steady/linear Stokes fluid model. Following this approach the flow rate boundary condition (19) is regarded as a constraint for the solution of the fluid problem. As such it is enforced by a Lagrange multiplier approach in a way similar to the incompressibility. Being a scalar constraint, we need a scalar multiplier $\lambda$, resulting in the following weak formulation: Find $\boldsymbol{u} \in V, p \in Q$ and $\lambda \in \mathbb{R}$ such that for all $\boldsymbol{v} \in V, q \in Q$ and $\psi \in \mathbb{R}$, it holds

$$
\begin{cases}\mu\left(\nabla \boldsymbol{u}+(\nabla \boldsymbol{u})^{T}, \nabla \boldsymbol{v}\right)-(p, \nabla \cdot \boldsymbol{v})+\lambda \int_{\Gamma} \boldsymbol{v} \cdot \boldsymbol{n} d \gamma & =F(\boldsymbol{v}) \\ (q, \nabla \cdot \boldsymbol{u}) & =0 \\ \psi \rho_{f} \int_{\Gamma} \boldsymbol{u} \cdot \boldsymbol{n} d \gamma & =\psi Q\end{cases}
$$

where $V=\left\{\boldsymbol{v} \in\left(H^{1}\left(\Omega_{f}\right)\right)^{3}:\left.\boldsymbol{v}\right|_{\Sigma_{D}}=\mathbf{0}\right\}, \Sigma_{D}$ being the portion of the boundary where Dirichlet conditions are prescribed, $Q=L^{2}(\Omega), F$ accounts for possible non-homogeneous Dirichlet and/or Neumann conditions on $\partial \Omega_{f} \backslash \Gamma$, and $(\cdot, \cdot)$ denotes the $L^{2}$ inner product.

It is possible to prove that beyond the flow rate condition (19)

1. the augmented formulation prescribes a constant traction on $\Gamma$ aligned with its normal direction;

2. the constant coincides with the Lagrange multiplier $\lambda$,

that means

$$
-p \boldsymbol{n}+\mu\left(\nabla \boldsymbol{u}+(\nabla \boldsymbol{u})^{T}\right) \boldsymbol{n}=\lambda \boldsymbol{n} \quad \text { on } \Gamma .
$$

From the quantitative view point, the overall result is that a constant-in-space traction is prescribed (the constant being unknown) resulting in a less stringent condition than the Dirichlet one of the empirical approach. This method is particularly suited when the artificial cross section 
is orthogonal to the longitudinal axis, so that vector $\boldsymbol{n}$ is truly aligned along the axial direction. Generalization of this strategy to the unsteady/non-linear case can be found in [211], the unknown Lagrange multiplier variable being a function of time $(\lambda=\lambda(t))$. Inf-sup condition for the twofold saddle point problem (22) is proved [211].

For the numerical solution of this formulation, one could rely either on a monolithic strategy where the full augmented matrix is built and solved, or on splitting techniques. In particular, in $[66,211]$ it is proposed to write the Schur complement scalar equation with respect to the Lagrange multiplier, leading to an algorithm where two standard fluid problems with Neumann conditions on $\Gamma$ need to be solved at each time step (exact splitting technique). The latter approach preserves modularity, that is it could be implemented using available standard legacy fluid solvers in a black box fashion. This is an interesting property in view of the application to cases of real interest, as done e.g. in [220, 217, 168, 91].

To reduce the computational time needed by the exact splitting approach, in [212] a different splitting procedure is proposed, which introduces an error near the boundary that is however always remarkably smaller than the one produced by the empirical approach. This strategy (called inexact splitting technique) requires the solution of just one standard fluid problem at each time step, thus halving the computational time with respect to that of the exact splitting technique.

To extend the augmented approach to the case of $n$ flow rate conditions one Lagrange multiplier has to be introduced for each condition. In this case, the exact splitting procedure requires the solution of $n+1$ fluid problems at each time step (see [66]), whereas the inexact procedure still needs to solve just one fluid problem.

Another approach, holding for the case of single as well as multiple flow rate conditions, is to perform an appropriate factorization of the augmented algebraic system that allows to reduce the computational costs with no extra errors, as recently addressed in [144].

The augmented formulation has been extended to the quasi-Newtonian case in [59].

Augmented formulation: compliant walls.. The extension of the augmented formulation to the case of compliant walls is addressed in [74]. There are basically two strategies we may consider. In the former ("split-then-augment") we first split the fluid-structure interaction problem in a segregated way, so to apply the augmentation procedure to the fluid subproblem at each iteration of the partitioned algorithm. In this case, one of the approaches described for the rigid case can be applied straightforwardly. This method is successfully considered in real settings in [173].

In the latter strategy ("augment-then-split") we directly perform the augmentation on the FSI problem. At the numerical level, we still have the option of pursuing either a monolithic or a partitioned approach. In the former case, suitable preconditioners are mandatory [50]. In the latter one, the problem can be formally reduced to the Schur complement equation for the sole $\lambda$. This actually implies at each time step the solution of two standard FSI problems with Neumann conditions on $\Gamma$, thus preserving modularity with respect to available FSI legacy solvers [74].

\subsubsection{Mean pressure boundary conditions}

To prescribe the mean pressure condition (20) we can follow an approach similar to the empirical one, where a velocity profile is arbitrarily selected to fulfill the given flow rate. In this case, we can postulate that the pressure on $\Gamma$ is constant and that the normal viscous stress can be discarded, so that

$$
p \boldsymbol{n}-\mu\left(\nabla \boldsymbol{u}+(\nabla \boldsymbol{u})^{T}\right) \boldsymbol{n}=P \boldsymbol{n} \quad \text { on } \Gamma .
$$


The previous assumption is generally acceptable because the pressure changes in arteries mainly occur along the axial direction.

This approach results in the prescription of a standard Neumann condition, being the average pressure considered as the boundary traction. In the process of numerical discretization, for $P=0$ it requires no further action than just assembling the matrix for homogeneous Neumann conditions. Therefore this has been called "do-nothing" approach [97]. This name is suggestive, however it has to be kept in mind that an "action" is actually performed (even if implicitly) [210, 209]. For instance, for the Stokes problem, the do-nothing approach corresponds to the following weak formulation (for the sake of simplicity we still refer to the steady case): Find $\boldsymbol{u} \in V$ and $p \in Q$ such that for all $\boldsymbol{v} \in V$ and $q \in Q$, it holds

$$
\begin{cases}\mu\left(\nabla \boldsymbol{u}+(\nabla \boldsymbol{u})^{T}, \nabla \boldsymbol{v}\right)-(p, \nabla \cdot \boldsymbol{v}) & =-P \int_{\Gamma} \boldsymbol{v} \cdot \boldsymbol{n} d \gamma+F(\boldsymbol{v}), \\ (q, \nabla \cdot \boldsymbol{u}) & =0,\end{cases}
$$

where we have used the same notation introduced for the augmented formulation (22). Even if (23) is an approximation, for a section $\Gamma$ orthogonal to the longitudinal direction the contribution $\int_{\Gamma} \mu\left(\nabla \boldsymbol{u}+(\nabla \boldsymbol{u})^{T}\right) \boldsymbol{n} d \gamma$ is in general expected to be very small if compared to $\int_{\Gamma} p d \gamma$.

Besides the grad-grad formulation (24), other weak formulations may be considered as well for the do-nothing approach, for example the curl-curl formulation having the pressure as natural condition. In this case, the do-nothing approach corresponds to the boundary condition

$$
p=P \quad \text { on } \Gamma,
$$

the only assumption being that the pressure is constant on $\Gamma$, without any further request on the smallness of the viscous boundary term (see [47, 210, 211]).

Remark 1. In principle, also mean pressure conditions (20) can be enforced using a Lagrange multiplier approach, as done for the flow rate problem. In a somehow dual situation to the case of flow rate conditions, the Lagrange multiplier in this case represents the normal velocity to the section $\Gamma$ and the augmented approach is implicitly forcing it to be constant in space. While a constant pressure over $\Gamma$ is an acceptable approximation, the same is not true for a normal constant velocity. For this reason, the augmented Lagrange multiplier approach for mean pressure conditions does not represent a reliable option.

Remark 2. A do-nothing formulation for the flow rate conditions is possible too, see [97, 210]. As a matter of fact, this was the first attempt to provide a mathematically sound formulation to the flow rate problem. This approach relies on the introduction of a set of functions that represents the lifting of the flow rate data inside the domain, in a way similar to what is done for standard Dirichlet conditions. See also [47] for a curl-curl formulation. However, the lifting functions, called flux-carriers, are not easy to construct in general. Because of that, the do-nothing approach for flow rate conditions is not very popular. Nevertheless, it is worth noting that this formulation implies that the traction on $\Gamma$ is constant and aligned with its normal - see [210] - as for the solution found with the Lagrange multiplier approach. We argue therefore that the do-nothing approach for flow rate conditions computes the same solution found by the augmented formulation (22).

Remark 3. A popular weak formulation of the incompressible Navier-Stokes equations discards the divergence free term $(\nabla \boldsymbol{u})^{T}$ in the definition of the fluid Cauchy stress tensor (2), so for a 
constant viscosity we have $\nabla \cdot\left(\mu(\nabla \boldsymbol{u})^{T}\right)=0$. This allows to have a block-diagonal pattern in the corresponding Finite Element stiffness matrix arising after space discretization. It is pointed out in [97] that with this simplified formulation, the associated do-nothing condition at $\Gamma, p \boldsymbol{n}-$ $\mu \nabla \boldsymbol{u} \boldsymbol{n}=$ Pn, is more appropriate for artificial boundaries than (23), since it computes correctly the Poiseuille solution - as opposed to the case with the term $(\nabla \boldsymbol{u})^{T}$. In [210, 209] it is however pointed out how mixed Dirichlet/Neumann conditions at the artificial boundary (prescribing Neumann conditions only in the normal direction and homogeneous Dirichlet in the tangential ones) can correctly capture the Poiseuille solution even with the complete formulation of the fluid stress tensor.

\subsubsection{A control-based approach}

A different strategy for the fulfillment of condition (19) is based on the minimization of the mismatch functional

$$
J(\boldsymbol{v})=\frac{1}{2}\left(\int_{\Gamma} \boldsymbol{v} \cdot \boldsymbol{n} d \gamma-Q\right)^{2}
$$

constrained by the fact that $\boldsymbol{v}$ satisfies the incompressible Navier Stokes equations [73].

This strategy is somehow the dual of the augmented strategy, where the defective boundary data were considered as a constraint to the energy minimization of the fluid. Instead, in the current approach the aim is to minimize (25) with the constraint given by the fluid equations. The constrained minimization problem leads to the Karush-Kuhn-Tucker (KKT) conditions, whose numerical solution can be obtained by iterative algorithms. In particular in [73] the normal traction on $\Gamma$ is used as control variable for the minimization of the mismatch functional.

In [74], this approach is considered for the compliant case. In particular, a fast algorithm based on a convenient combination of the iterations of the partitioned FSI solver and of the constrained minimization has been introduced. In $[122,80,81]$, the extensions to the non-Newtonian, quasiNewtonian, and visco-elastic cases are addressed.

The same approach can also be used to fulfill the mean pressure conditions (20) over one or several boundary sections. This allows to prescribe a mean pressure condition on a section oblique with respect to the longitudinal axis too. In this case the control variable is the complete traction vector, that is also the direction of the traction is a priori unknown [73].

Two-dimensional numerical results highlight the accuracy of the control-based approach for the prescription of both defective conditions (19) and (20) at a computational cost which is comparable with the one featured by the exact splitting strategy introduced to solve the augmented problem.

Remark 4. The above constrained minimization approach resembles variational methods of Data Assimilation - see e.g. [54, 55, 19, 213]. In this variational context, a further possibility consists of including in (25) some sparse measures available not only on the boundary but also inside the region of interest. The aim could be to either driving the solution to match (in some sense) these data [55], possibly including stochastic information on the measurement error to quantify the uncertainty affecting the final assimilation [56], or obtaining a better estimate of one or more parameters of physical interest, e.g. the wall compliance [162, 20, 225].

\subsubsection{Further developments and comments}

A defective Nitsche-like approach.. The original idea of Nitsche of prescribing Dirichlet conditions with a penalization approach [146] has been recently extended to the case of flow rate boundary 
conditions in [227]. This strategy does not augment the variables of the original problem, however it introduces a parameter for the penalization that needs to be properly tuned. In addition, it deals with non standard bilinear forms that require ad hoc implementation. Two dimensional numerical results shown in [227] highlight the accuracy of the method, whereas three-dimensional results reported in [172] demonstrate that this is an effective approach for real applications. A similar approach has been extended to fulfill the mean pressure condition (20) and the FSI case in [214].

Defective boundary conditions for the structure. In some circumstances, boundary information available on the (artificial) arterial cross section (indicated by the circular annulus $\Gamma_{s}$ in Figure 9) might be insufficient to provide the boundary conditions that are required to "close" the structure problem. This is e.g. the case where the only data available is the area of the cross-sectional lumen

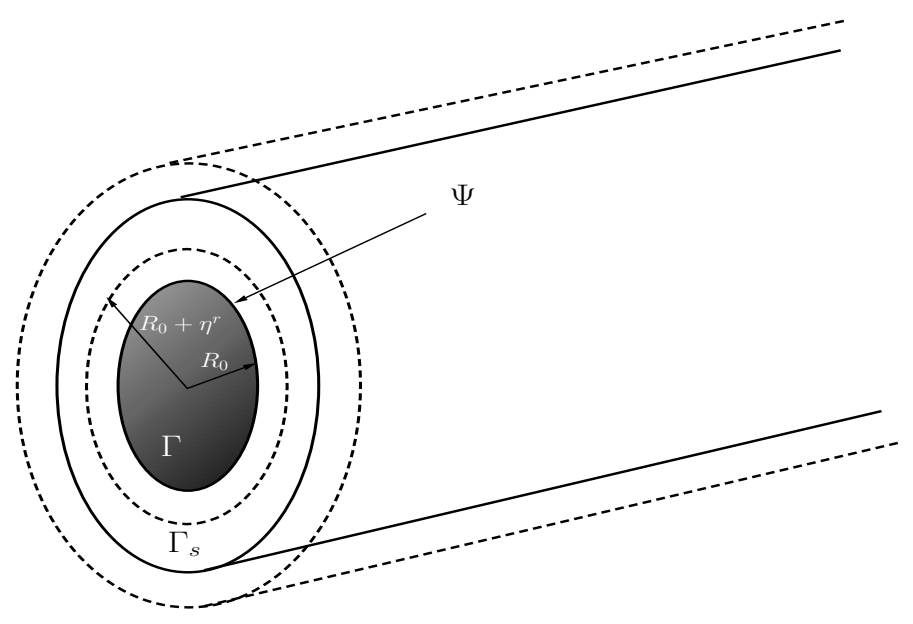

Figure 9: Artificial section $\Gamma_{s}$ for the structure problem.

$\Gamma$, that is the real number (function of $t$ ) $A(t)$. In this case we have

$$
\int_{\Gamma(t)} r d r d \varphi=\int_{0}^{2 \pi} \int_{0}^{R^{0}(\varphi)+\widetilde{\eta}^{r}(t, \varphi)} r d r d \varphi=A(t),
$$

where $r$ and $\varphi$ are the radial and angular coordinates respectively. Here $R^{0}$ is the distance of a point of the line $\Psi=\partial \bar{\Gamma}_{s} \cap \partial \bar{\Gamma}$ from the center of mass of $\Gamma^{0}$, and $\eta^{r}$ is the radial displacement of the structure. This may be read as an average condition on the displacement (formulated in polar coordinates) of the line $\Psi$.

The single (scalar) condition (26) is (by far!) incomplete to close the structural problem (1e) in $\Gamma_{s}$ (note that Dirichlet or Neumann conditions would provide three conditions on every point of $\left.\Gamma_{s}\right)$. We are therefore dealing with a situation of defective boundary conditions for the structure, similarly to those already faced for the fluid problem.

Proceeding as for the empirical approach to prescribe flow rate conditions (that were recast in pointwise conditions by an assumption on the velocity profile), we can here assume that the radial displacement at the line $\Psi$ is independent of $\varphi[69,109]$. To reduce the impact of the arbitrary 
assumption on the shape of the section, moving along similar lines as for the flow rate conditions, a control-based approach can be pursued based on the minimization of an appropriate mismatch functional [71] .

On the prescription of defective Robin conditions.. Often, in computational hemodynamics one has to deal with the following defective Robin boundary condition

$$
R \int_{\Gamma} \boldsymbol{u} \cdot \boldsymbol{n} d \gamma+\frac{1}{|\Gamma|} \int_{\Gamma} p d \gamma=M
$$

obtained by a linear combination of conditions (19) and (20); $M$ is a given scalar function of time, and $R$ is a parameter that can be either measured or set up to damp spurious reflections induced by the truncation of the domain of interest $[219,150,147,109]$.

The prescription of such a condition for $R \in(0,+\infty)$ is discussed in [219], where a monolithic approach has been proposed, in [75] for the case of the augmented and control-based formulations, and in [214] for the Nitsche strategy.

Remark 5. Using Neumann conditions at the downstream artificial sections might induce numerical instabilities. These are usually called backflow instabilities because they are typically triggered by the backflow in diastole at the outlet sections of a vascular district, see [41, 42, 16, 138]. In this case, using a condition like (27) will "cure" the problem by setting the (nonlinear) parameter $R$ to be active only in the presence of a normal velocity component incoming the domain.

Defective conditions alternative to the mean pressure.. Another possible defective condition prescribes the average traction (in place of the average pressure as done in (20)):

$$
\frac{1}{|\Gamma|} \int_{\Gamma} \boldsymbol{T}_{f}(\boldsymbol{u}, p) \boldsymbol{n} \cdot \boldsymbol{n} d \gamma=T,
$$

for a given function of time $T(t)$. Its actual implementation follows closely that of condition (20). In particular, if a do-nothing approach is considered for (28), one obtains again formulation (24), which still actually prescribes condition (23), with the right hand side $P$ replaced by $T$. However, in this case condition (23) is consistent with the defective condition (28) (unlike (20), see Sect. 3.1.2).

The optimal control approach can be applied for the prescription of (28) provided that a suitable functional is considered. In [74], numerical results highlight the differences between the solutions obtained with this strategy to prescribe condition (20) and (28). We observe that also the Nitsche-like method could be extended to prescribe condition (28), as shown in [214] .

In a similar way, given two functions of time $P_{t o t}(t)$ and $T_{t o t}(t)$, we can consider defective conditions involving the total pressure

$$
\frac{1}{|\Gamma|} \int_{\Gamma} p_{t o t} d \gamma=P_{t o t},
$$

or the mean total normal traction

$$
\frac{1}{|\Gamma|} \int_{\Gamma} \boldsymbol{T}_{f}\left(\boldsymbol{u}, p_{t o t}\right) \boldsymbol{n} \cdot \boldsymbol{n} d \gamma=T_{t o t},
$$


where

$$
p_{t o t}=p+\frac{\rho_{f}}{2}|\boldsymbol{u}|^{2}
$$

denotes the total pressure. Notice that using the identity

$$
(\boldsymbol{u} \cdot \nabla) \boldsymbol{u}=\frac{1}{2} \nabla|\boldsymbol{u}|^{2}+\operatorname{curl} \boldsymbol{u} \times \boldsymbol{u},
$$

the natural boundary conditions associated with the Navier-Stokes problem involve the total pressure or the total traction. The do-nothing procedure can be performed in these cases as done for the average pressure and traction (see, e.g., $[209,210,69]$ ).

Applications of clinical interest.. We conclude this section by mentioning some practical applications of the strategies described above for the treatment of defective boundary conditions, in particular the one based on the augmented approach.

This strategy has been successfully applied to investigate the jet impinging the ascending aortic wall downstream a bicuspid aortic valve [220,217], or in an abdominal aortic aneurysm to assess the viscous forces [168].

Another application is proposed in [170] to improve approximation of flow rate measurements obtained by ultrasound devices. Doppler velocimetry is currently used to provide an estimate of the flow rate in a vascular district starting from the measurement of the maximum velocity. This estimate is based on the assumption of parabolic profile (Doucette formula). Despite its popularity and simplicity, this formula relies on an unrealistic hypothesis, that of parabolic profile, which makes sense only for steady flows in rectilinear circular pipes. The basic idea in [170] is to extend the Doucette formula including the dependence of the flow rate also on the pulsatility, by means of the Womersley number. The fitting of the new formula is performed by running several numerical simulations in different scenarios by applying the flow rate with the augmented approach. This choice is motivated by the necessity of finding a relation between flow rate and maximum velocity not biased by an a priori choice of the velocity profile. Results prove the reliability of this method, which has then been successively validated in clinical settings [216, 171].

\subsection{The role of the Riemann variables for $1 D$ models}

When solving propagative equations like the 1D Euler system (7), a major problem is the correct treatment of the boundary conditions. For instance, when the end points do not correspond to physical boundaries, non-reflecting conditions should be prescribed to the original continuous problem. With this we mean conditions that correctly describe the motion of the different propagative components, without introducing any spurious effect like unphysical reflections. Moreover, after space discretization, the number of equations at the boundary points is not enough to close the system at the algebraic level. This demands for more numerical boundary conditions than those required by the original problem itself.

In this regard, a key concept is that of Riemann variables. We illustrate the role of Riemann variables on the single arterial tract represented by the 1D system (7). Let us introduce the following notation: $L=\left[\begin{array}{c}\boldsymbol{l}_{1}^{T} \\ \boldsymbol{l}_{2}^{T}\end{array}\right], R=\left[\begin{array}{ll}\boldsymbol{r}_{1} & \boldsymbol{r}_{2}\end{array}\right]$, where $\boldsymbol{l}_{1}, \boldsymbol{l}_{2}$ and $\boldsymbol{r}_{1}, \boldsymbol{r}_{2}$ are the left and right eigenvectors of $H$. We can write therefore $H=R \Lambda L$, with $\Lambda=\operatorname{diag}\left(\lambda_{1}, \lambda_{2}\right)$. In addition, we define the Riemann variables $W_{1}$ and $W_{2}$ as

$$
\frac{\partial W_{1}}{\partial \boldsymbol{U}}=\boldsymbol{l}_{1}, \quad \frac{\partial W_{2}}{\partial \boldsymbol{U}}=\boldsymbol{l}_{2} .
$$


These are also called characteristic variables. Notice that, depending on the vessel law and on the assumptions on the parameters of the system, an explicit expression of the characteristic variables as a function of the physical variables

$$
W_{i}=\zeta_{i}(A, Q), i=1,2,
$$

can be derived. For example, in the case $\alpha=1$ (with $\alpha$ the Coriolis coefficient, see Section 2.2.1) and for the algebraic law (11) we have [67]

$$
W_{1,2}=\zeta_{1,2}(A, Q)=\frac{Q}{A} \pm 4 \sqrt{\frac{\beta}{2 \rho_{f} A_{0}}} A^{1 / 4}
$$

where $\beta$ is given by (11).

After multiplication of (7) by $L$, we have the characteristic system

$$
\frac{\partial \boldsymbol{W}}{\partial t}+\Lambda \frac{\partial \boldsymbol{W}}{\partial z}+\boldsymbol{G}(\boldsymbol{W})=\mathbf{0} \quad z \in(0, L), t>0,
$$

with $\boldsymbol{G}=L \boldsymbol{B}$. Componentwise, these equations read

$$
\frac{\partial W_{i}}{\partial t}+\lambda_{i} \frac{\partial W_{i}}{\partial z}+G_{i}\left(W_{1}, W_{2}\right)=0, \quad i=1,2 .
$$

After introducing the characteristic lines $z_{i}(t)$ for $i=1,2$, solution of the problems

$$
\frac{d z_{i}}{d t}=\lambda_{i}, \quad i=1,2
$$

our problem reads

$$
\left.\frac{d W_{i}}{d t}\right|_{z=z_{i}(t)}+G_{i}\left(W_{1}, W_{2}\right)=0 .
$$

When $\boldsymbol{G}=\mathbf{0}$ we have two independent problems, the $W_{i}$ 's are constant along their respective characteristic line and they are called Riemann invariants.

As we noticed in (10), the diagonal entries of $\Lambda$ are $\lambda_{1}>0$ and $\lambda_{2}<0$. This means that the Riemann variable $W_{1}$ propagates forward along $z$, while $W_{2}$ is moving backward. Otherwise said, $z=0(z=L)$ is an inflow end point for $W_{1}\left(W_{2}\right)$, so we need one boundary condition at each end point.

A first possibility is given by prescribing conditions on the primary variables to system (7), i.e. the flow rate $Q=Q_{m}(t)$ or the area $A=A_{m}(t)$ (or equivalently the pressure).

Alternatively, one could consider the linearized system (33) and prescribe one of the characteristic variables $W_{i}$ so as to obtain a non-reflecting condition. In particular, following [95, 204] by the theory of characteristics we have

$$
\frac{\partial W_{1}}{\partial t}+R_{1}\left(W_{1}, W_{2}\right)=0 \quad \text { at } \quad z=0, \quad \frac{\partial W_{2}}{\partial t}+R_{2}\left(W_{1}, W_{2}\right)=0 \quad \text { at } \quad z=L,
$$

where $R_{i}=\boldsymbol{l}_{i}^{T} \cdot \boldsymbol{S}$, and $\boldsymbol{S}$ has been introduced in (9). For $R_{1}=0$ at $z=0$ and $R_{2}=0$ at $z=L$, the absorbing conditions reduce to $W_{1}=$ const at $z=0$ and $W_{2}=$ const at $z=L$ (with the constants given by the initial conditions) $[67,160]$. 
A third strategy consists in prescribing non-reflecting conditions directly to system (7). In this case, thanks to (32), a non-linear combination of the physical variables is prescribed.

Alternative boundary conditions at the outlets are provided by the structured tree modeling mentioned in Sect. $2.3[153,48]$, which assumes that the downstream vascular tree has a simple structure subject to suitable scaling laws. This allows to derive suitable relations between flow rate and pressure at the outlets.

Another problem in the management of boundary conditions is that at the numerical level, after space discretization, an additional boundary equation is needed at each boundary to close the algebraic system. This extra-condition needs to be extracted by the problem itself to avoid inconsistencies of the solution with the physical problem. A possible workaround is offered by the so-called compatibility conditions, that provide a condition on the outgoing characteristic variable and are basically obtained by the projection of the equation along the eigenvectors corresponding to the outgoing characteristics in the boundary points,

$$
\begin{array}{ll}
\boldsymbol{l}_{2}^{T} \cdot\left(\frac{\partial \boldsymbol{U}}{\partial t}+H(\boldsymbol{U}) \frac{\partial \boldsymbol{U}}{\partial z}+\boldsymbol{B}(\boldsymbol{U})\right)=0 & z=0, t>0 \\
\boldsymbol{l}_{1}^{T} \cdot\left(\frac{\partial \boldsymbol{U}}{\partial t}+H(\boldsymbol{U}) \frac{\partial \boldsymbol{U}}{\partial z}+\boldsymbol{B}(\boldsymbol{U})\right)=0 & z=L, t>0 .
\end{array}
$$

In terms of characteristic variables, this corresponds for each time interval $\left[t^{n}, t^{n+1}\right]$ to the following system of ODE localized at the boundaries

$$
\begin{aligned}
& \frac{d}{d t} W_{i}\left(t, y_{i}(t)\right)+G_{i}\left(W_{1}, W_{2}\right)=0, \quad i=1,2 \quad t \in\left[t^{n}, t^{n+1}\right] \\
& y_{1}\left(t^{n+1}\right)=L, \quad y_{2}\left(t^{n+1}\right)=0 .
\end{aligned}
$$

If $G_{i}=0$, the solution of this system of ODE could be obtained by tracing back the characteristic lines exiting the domain and imposing that the corresponding characteristic variable is constant, yielding

$$
W_{2}\left(t^{n+1}, 0\right)=W_{2}\left(t^{n},\left|\lambda_{2}^{n}(0)\right| \Delta t\right), \quad W_{1}\left(t^{n+1}, L\right)=W_{1}\left(t^{n}, L-\lambda_{1}^{n}(L) \Delta t\right),
$$

see Figure 10. These numerical-driven conditions together with the ones prescribed by the mathematical problem form a non-linear system in the unknowns $\left.A^{n+1}\right|_{z=0},\left.Q^{n+1}\right|_{z=0},\left.A^{n+1}\right|_{z=L},\left.Q^{n+1}\right|_{z=L}$.

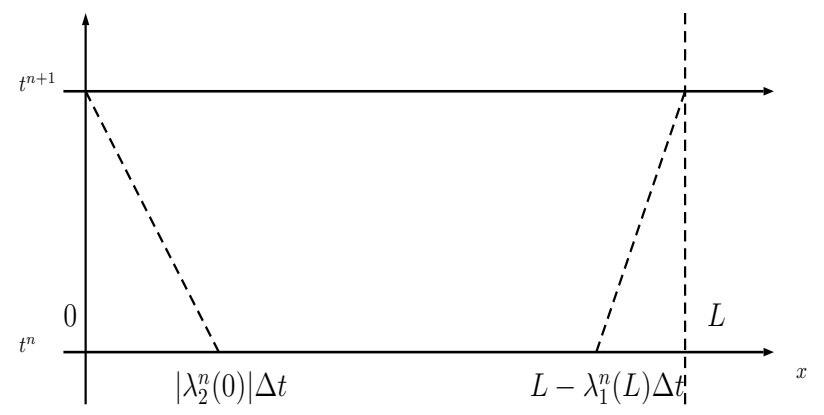

Figure 10: Characteristic variables extrapolation in the case $\boldsymbol{G}=\mathbf{0}$.

Finally, we mention the treatment of matching conditions at the bifurcations. This requires supplementing the interface matching conditions (13) with further compatibility conditions. To 
find them out, referring to Figure 3 , let $W_{j}^{i}$ be the $j$-th characteristic variable in the branch $\mathcal{B}^{i}$ at the bifurcation point. For ease of notation, the temporal index is understood. The characteristic variables at the bifurcation point are unknowns, so that at each bifurcation we have 12 unknowns, namely $Q^{i}, i=1,2,3, A^{i}, i=1,2,3, W_{j}^{i}, i=1,2,3, j=1,2$. The physical conditions (13) provide 3 relations, and 6 relations are provided by (32), namely $W_{j}^{i}=\zeta_{j}^{i}\left(A^{i}, Q^{i}\right), j=1,2, i=1,2,3$. To close the system we need 3 more relations at the inlet of $\mathcal{B}^{1}$ and at the outlet of $\mathcal{B}^{2}$ and $\mathcal{B}^{3}$ given, e.g., by the compatibility conditions obtained by extrapolating the outgoing characteristic variables. This leads to a closed non-linear $12 \times 12$ system for each bifurcation.

\subsection{OD models and the enforcement of boundary data}

As observed in Section 2.3, when deriving a 0D model from a 1D one (and, in fact, from a 3D one) the explicit spatial dependence is lost in the process of model reduction. This may require some attention when coupling two heterogeneous models, in particular for the derivation and treatment of the interface conditions, as discussed in Section 4.2.

As a matter of fact, we need to localize quantities at the interface that were formerly "delocalized" because of the model reduction process. As we have pointed out, in our derivation procedure, a lumped parameter model of an arterial tract is not just a reduction of the NavierStokes equations, but of the whole boundary value problem (including therefore the associated boundary conditions). This was reflected by the different configurations we have found for the same arterial tract. In particular, the $\mathcal{L}$ configuration implicitly postulates that $\widehat{Q}_{p}$ and $\widehat{P}_{d}$ are input variables or, with a little abuse of notation, "boundary" data. Correspondingly, $\widehat{P}_{p}$ and $\widehat{Q}_{d}$ are state variables (henceforth unknown) that are computed by the model to characterize the dynamics in the tract. Similarly, we notice that (see Fig. 5)

1. $\widehat{P}_{p}$ and $\widehat{Q}_{d}$ are "boundary data" for the $\mathcal{L}$-inverted network;

2. $\widehat{Q}_{d}$ and $\widehat{Q}_{p}$ are "boundary data" for the $\pi$ network;

3. $\widehat{P}_{d}$ and $\widehat{P}_{p}$ are "boundary data" for the $\mathcal{T}$ network.

The different configurations therefore incorporate the variables that need to be prescribed to solve the associated ordinary differential system. Going back to the very root of our derivation - a volume averaging of the Navier-Stokes equations - we may say that the different network topologies reflect different boundary value problems. When we assume flow rate as boundary data, this is corresponded by velocity (Dirichlet) boundary conditions for the original 3D problem. Instead, traction (Neumann) conditions are corresponded by pressure data in the lumped parameter model.

This analysis on the role of the interface variables will set up the guidelines for the interface treatment in case coupled models involving 0D models (thus 1D-0D or 3D-0D) will be solved numerically by means of iteration-by-subdomain algorithms, as we will see in Section 5.1.

\section{Coupling of 3D-1D, 3D-0D, and 1D-0D models}

Once suitable stand-alone models are available to describe some portions of the cardiovascular system with different geometric details, we are ready to build geometric multiscale models obtained by their coupling. In practice, the use of geometric multiscale models allows the simulation of the whole circulatory system (or at least of all of its most relevant elements) with low dimensional models on the background and higher dimensional high fidelity models for those regions where a very precise numerical investigation is desired. 
In particular, here we address the problems obtained by coupling 3D and 1D (Sect. 4.1), 3D and 0D (Sect. 4.2), and 1D and 0D (Sect. 4.3) models.

We illustrate our approach in the case of a cylindrical vessel. We follow two steps:

1. domain splitting: we partition the cylindrical vessel into two non overlapping regions, called subdomains, and write the 3D/FSI problem over the two regions coupled by appropriate interface conditions;

2. dimensional model reduction: the 3D/FSI problem in one of the two subdomains (the downstream one in our case) is downscaled to either a 1D (Sect. 4.1) or a 0D (Sect. 4.2) model; the interface conditions are adapted correspondingly for the new coupled problem. For the sake of reducing further the computational cost, in Sect. 4.3 we reduce the upstream 3D model to a $1 \mathrm{D}$ problem, to be coupled with a $0 \mathrm{D}$ model downstream.

Coupling occurs at interfaces corresponding to a cross section for the $3 \mathrm{D}$ case, to a single point for the $1 \mathrm{D}$ model, and to the proximal region for the $0 \mathrm{D}$ model. The prescription of appropriate interface conditions and their accurate numerical approximation clearly represent a crucial issue.

For the sake of notation, in what follows we add the subscript $n D, n=0,1,3$ to the quantities relevant for the corresponding problems.

For AN ABSTRACT DERIVATION OF COUPLED DIMENSIONALLY HETEROGENEOUS PROBLEMS, WE REFER THE READER TO [24].

\subsection{D-1D coupling}

\subsubsection{Formulation of the coupled problem: domain decomposition and reduction}

Let us consider the FSI problem (1) in the 3D cylindrical domain $\bar{\Omega}=\bar{\Omega}_{f} \cup \bar{\Omega}_{s}$ depicted in Figure 11, top, together with initial conditions and boundary conditions at the proximal and distal boundaries $\Sigma_{\text {prox }}$ and $\Sigma_{\text {dist }}$, respectively (the subscript $f$ stands for fluid and $s$ for structure), and at the lateral structure $\Sigma_{\text {ext }}$. In short we denote (1) as

$$
\mathcal{P}_{3 D}\left(\boldsymbol{u}_{3 D}, p_{3 D}, \boldsymbol{\eta}_{3 D} ; \Omega\right)=0,
$$

together with the specified boundary and initial conditions.

We split $\Omega$ into two subdomains, $\Omega_{1}$ and $\Omega_{2}$, and call $\Gamma=\Gamma_{f} \cup \Gamma_{s}$ their common interface, $\Gamma_{f}$ being the interface for the fluid subdomain and $\Gamma_{s}$ the interface for the structure one (see Figure 11, center). Then, problem (35) is equivalently rewritten as

$$
\begin{array}{ll}
\mathcal{P}_{3 D}\left(\boldsymbol{u}_{3 D}^{1}, p_{3 D}^{1}, \boldsymbol{\eta}_{3 D}^{1} ; \Omega_{1}\right)=0, & \\
\boldsymbol{u}_{3 D}^{1}=\boldsymbol{u}_{3 D}^{2} & \text { on } \Gamma_{f}, \\
\boldsymbol{T}_{f}\left(\boldsymbol{u}_{3 D}^{1}, p_{3 D}^{1}\right) \boldsymbol{n}_{1}=\boldsymbol{T}_{f}\left(\boldsymbol{u}_{3 D}^{2}, p_{3 D}^{2}\right) \boldsymbol{n}_{2} & \text { on } \Gamma_{f} \\
\boldsymbol{\eta}_{3 D}^{1}=\boldsymbol{\eta}_{3 D}^{2} & \text { on } \Gamma_{s} \\
\boldsymbol{T}_{s}\left(\boldsymbol{\eta}_{3 D}^{1}\right) \boldsymbol{n}_{1}=\boldsymbol{T}_{s}\left(\boldsymbol{\eta}_{3 D}^{2}\right) \boldsymbol{n}_{2} & \text { on } \Gamma_{s}, \\
\mathcal{P}_{3 D}\left(\boldsymbol{u}_{3 D}^{2}, p_{3 D}^{2}, \boldsymbol{\eta}_{3 D}^{2} ; \Omega_{2}\right)=0, &
\end{array}
$$

together with the same boundary and initial conditions (reformulated for $\boldsymbol{u}_{3 D}^{i}, p_{3 D}^{i}, \boldsymbol{\eta}_{3 D}^{i}, i=1,2$ ) of (35). Here $\boldsymbol{n}_{j}, j=1,2$, is the unit vector normal to $\Gamma$ and outward to $\Omega_{j}$. We distinguish two types of interface conditions, kinematic ones (36b) and (36d), stating the continuity of the 
fluid velocity and of the structure displacement, and dynamic ones (36c) and (36e), stating the continuity of the tractions.

The equivalence between (35) and (36) is intended in the following sense: if $\boldsymbol{y}_{3 D}=\left(\boldsymbol{u}_{3 D}, p_{3 D}, \boldsymbol{\eta}_{3 D}\right)$ is a solution of problem (35), then $\left(\left.\boldsymbol{u}_{3 D}\right|_{\Omega_{j}},\left.p_{3 D}\right|_{\Omega_{j}},\left.\boldsymbol{\eta}_{3 D}\right|_{\Omega_{j}}\right), j=1,2$, are the solutions of (36). Conversely, if $\boldsymbol{y}_{3 D}^{1}$ and $\boldsymbol{y}_{3 D}^{2}$ solve problem (36), then the vector $\boldsymbol{y}_{3 D}$ such that $\left.\boldsymbol{y}_{3 D}\right|_{\Omega_{j}}=\boldsymbol{y}_{3 D}^{j}, j=1,2$, solves problem (35), see, e.g., $[178,71]$.

Successively we replace the distal 3D problem in $\Omega_{2}$ with a $1 \mathrm{D}$ model, while in $\Omega_{1}$ we retain the same 3D problem of (36a). In particular, by representing with $z \in[0, L]$ the longitudinal abscissa of the centerline of the cylinder $\Omega_{2}$, the corresponding 3D and $1 \mathrm{D}$ models will be coupled at the interface $\Gamma$ located at the point $z=0$ (see Fig. 11, bottom). Then, the 3D-1D coupled problem is given by coupling (36a) and (7), with prescribed initial conditions at $t=0$ and (i) boundary conditions at $\Sigma_{\text {prox }}^{1}$ and $\Sigma_{e x t}^{1}$ for the 3D subdomain; (ii) boundary conditions at $z=L$ for the 1D subdomain. These conditions may be selected as discussed in the previous sections. In addition, we need interface conditions to be prescribed on $\Gamma$. These conditions are critical for the well posedness and ultimately the reliability of the geometric multiscale model. In the next sections we elaborate this topic starting from the full conditions (36b-36e).

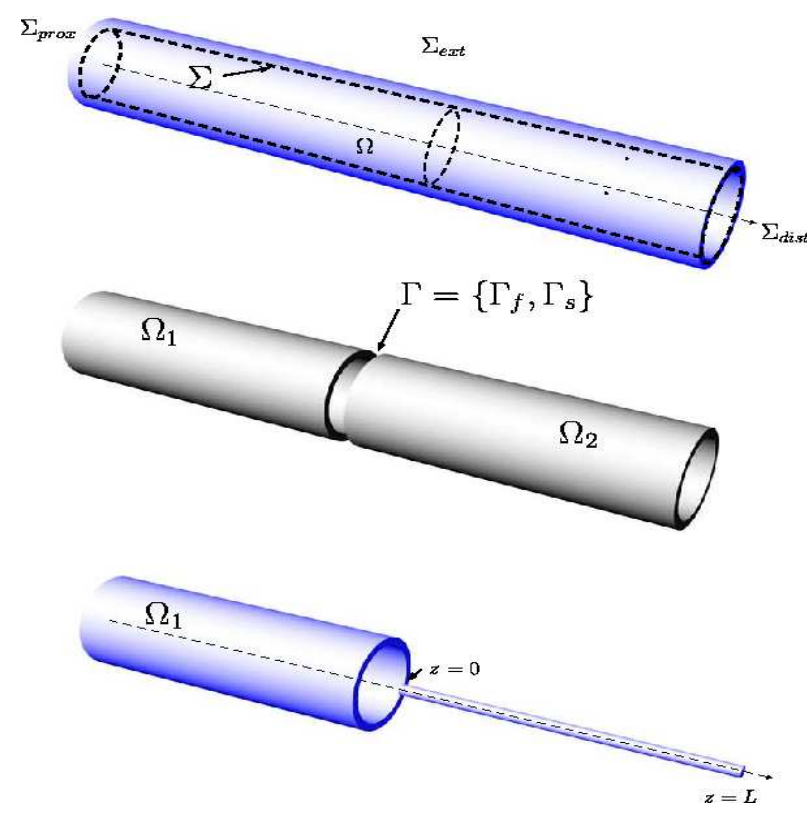

Figure 11: Schematic representation of the reference 3D-1D coupled model. A cylindrical domain at the top is split into two subdomains (center). The downstream one is eventually replaced by a $1 \mathrm{D}$ model (bottom).

\subsubsection{Interface coupling conditions}

The dimensional reduction of the downstream region introduces a mismatch between the two subdomains that is reflected by their interface conditions. The mismatch refers to the constitutive law for the structure problem postulated by the $1 \mathrm{D}$ model that is not necessarily the same as the one of the 3D upstream subdomain. In addition we have the geometrical mismatch due to the model 
reduction. The physical principles to obtain interface conditions aim at the preservation of both the kinematic and dynamic continuity conditions. Dimensional reduction prevents to prescribe the continuity of the full velocity field, since the 1D model computes only the average-in-space axial velocity (i.e. normal to the interface $\Gamma$ ) or equivalently the flow rate $Q$. Thus, kinematic condition (36b) reduces to

$$
\rho_{f} \int_{\Gamma_{f}} \boldsymbol{u}_{3 D} \cdot \boldsymbol{n} d \gamma=Q_{1 D}(z=0) .
$$

Concerning the wall displacement continuity condition (36d), we introduce the hypothesis of null displacement of the interface $\Gamma$ (and equivalently of the point located at $z=0$ ) along the axial direction. Moreover, we assume that the circumferential displacement is null, as it is reasonable in this context. Thus, the structure kinematic condition after the reduction gives information only on the $3 \mathrm{D}$ radial displacement, resulting in the condition

$$
\int_{0}^{2 \pi} \int_{0}^{R_{0}+\widetilde{\eta}_{3 D}^{r}\left(\Gamma_{s}, r=R_{0}, \varphi\right)} r d r d \varphi=A_{1 D}(z=0),
$$

where $\eta_{3 D}^{r}\left(\Gamma_{s}, r=R_{0}, \varphi\right)$ is the radial wall displacement at the interface $\Gamma_{s}$, evaluated at the line $\Psi=\bar{\Gamma}_{f} \cup \bar{\Gamma}_{s}$ (that is at $r=R_{0}$ ), as a function of the circumferential coordinate. Thus, condition (38) is, for the $3 \mathrm{D}$ problem, a defective condition for the radial displacement localized on the line $\Psi$.

WHEN CONSIDERING THE COUPLING OF 3D/FSI WITH 1D MODELS, WE SHOULD KEEP IN MIND THAT THE LATTER MODEL INTEGRATES IN ONE SYSTEM THE CONTRIBUTION OF BOTH FLUID AND STRUCTURE DYNAMiCs. Equation (6) ACTUALly DEFINES THE JOINT CONTRIBution to NORMAL (AXIAL) STRESSES OF THE CONTINUUM GIVEN BY FLUID AND STRUCTURE - COLLECTED UNDER THE NAME OF "PRESSURE". THIS IS IMPORTANT TO CORRECTLY WRITE INTERFACE CONDITIONS MATCHING (36c) AND (36e). As A MATTER OF FACT, WE CAN RIGOROUSLY WRITE AN INTEGRATED CONDITION FORMALLY EQUATING THE TRACTION OF THE FLUID+STRUCTURE CONTINUUM TO THE $1 \mathrm{D}$ NORMAL STRESS AT $z=0$. THIS YIELDS CONDITION

$$
\begin{gathered}
\frac{1}{\left|\Gamma_{f}\right|} \int_{\Gamma_{f}} \boldsymbol{T}_{f}\left(\boldsymbol{u}_{3 D}, p_{3 D}\right) \boldsymbol{n} \cdot \boldsymbol{n} d \gamma+\frac{1}{|\Psi|} \int_{\Psi} \boldsymbol{T}_{s}\left(\boldsymbol{\eta}_{3 D}\right) \boldsymbol{n} \cdot \boldsymbol{n} d \gamma=P_{1 D}(z=0) \\
=-P_{e x t}(z=0)-\psi\left(A_{1 D}(z=0), A_{0}(z=0), \boldsymbol{\beta}(z=0)\right),
\end{gathered}
$$

(WHERE THE NEGATIVE SIGN FOLLOWS FROM THE DIFFERENT ORIENTATION OF THE NORMAL VECTOR FROM THE TWO SIDES). THIS CONDITION IS CLEARLY INSUFFICIENT TO CLOSE THE 3D

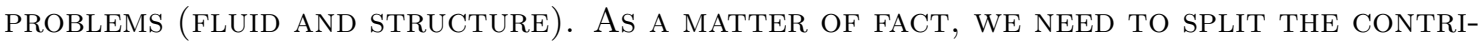
BUTIONS FOR FLUID AND STRUCTURE. HOWEVER, THIS INFORMATION CAN NOT BE PROVIDED BY THE 1D MODEL. A REASONABle NUMERICAL APPROACH CONSISTS IN A CONSISTENT SPLitTing BASED ON A PARAMETER $\theta \in[0,1]$ :

$$
\begin{aligned}
& \frac{1}{\left|\Gamma_{f}\right|} \int_{\Gamma_{f}} \boldsymbol{T}_{f}\left(\boldsymbol{u}_{3 D}, p_{3 D}\right) \boldsymbol{n} \cdot \boldsymbol{n} d \gamma=-\theta\left(P_{e x t}(z=0)+\psi\left(A_{1 D}(z=0), A_{0}(z=0), \boldsymbol{\beta}(z=0)\right)\right), \\
& \frac{1}{|\Psi|} \int_{\Psi} \boldsymbol{T}_{s}\left(\boldsymbol{\eta}_{3 D}\right) \boldsymbol{n} \cdot \boldsymbol{n} d \gamma=-(1-\theta)\left(P_{e x t}(z=0)+\psi\left(A_{1 D}(z=0), A_{0}(z=0), \boldsymbol{\beta}(z=0)\right)\right) .
\end{aligned}
$$




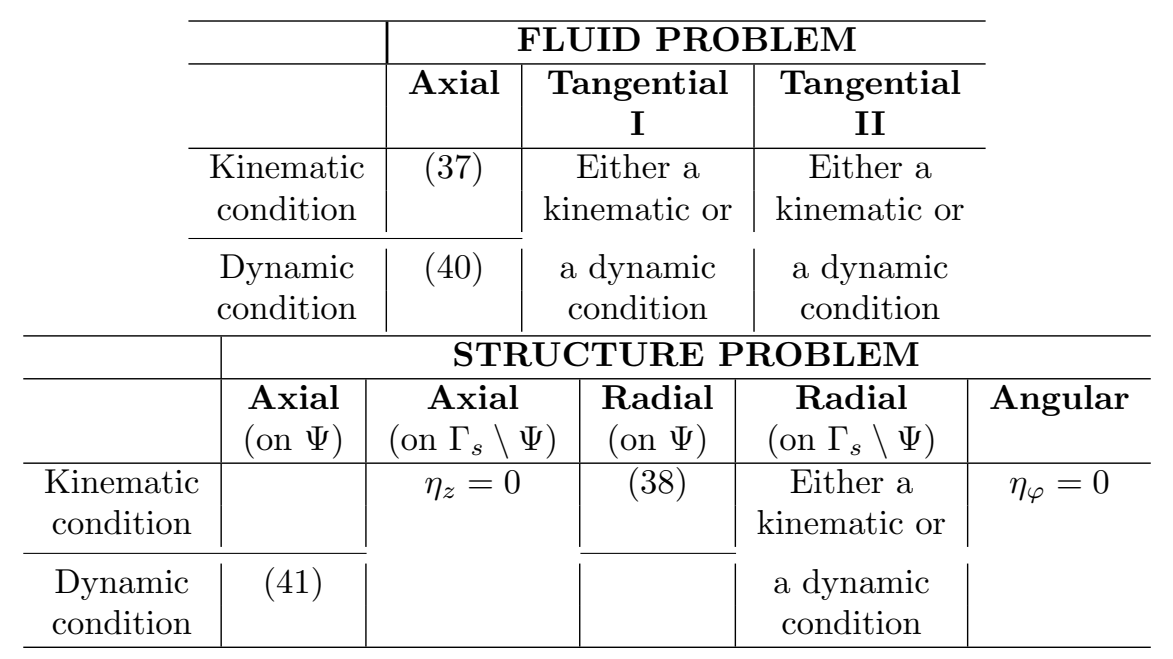

Table 2: Schematic representation of the available interface information in the 3D-1D coupling along the different coordinates and of the supplementary conditions requested. $\eta_{z}$ and $\eta_{\varphi}$ are the axial and angular structure displacement, respectively.

IF We SET $\theta=1$, We RECOVER the ClASSicAl APPROACH USED, E.G., IN [70], WHERE IN FACT THE 3D STRUCTURE IS NOT COUPled TO THE 1D PROBlem. ON THE CONTRARY, the CASE $\theta=0$ CORRESPONDS TO THE CASE OF A COLLAPSING TUBE IN $z=0$, WhEN The 3D Fluid and 1D problems are Detached. This is the CASe, E.G., OF the Jugular veins. More in General, We CAN EMPirically yet REASOnAbly assume the RUle $\theta=\frac{\left|\Gamma_{f}\right|}{\left|\Gamma_{f}\right|+\left|\Gamma_{s}\right|}$, Which Weights the Fluid and structure interface areas. Conditions (40)-(41) are in fact defective dynamic conditions in the axial direction.

Then, out of the 12 scalar pointwise continuity conditions (36b)-(36e) holding for the dimensionally homogeneous problem at each point of the interface $\Gamma$, only the 4 scalar averaged conditions $(37),(38),(40),(41)$ can be used for the 3D-1D coupling. For the 1D model they are enough to ensure well-posedness, whereas they are clearly insufficient for closing the $3 \mathrm{D}$ problem. We can therefore identify (a) coupling directions over $\Gamma$ where some information is exchanged, as well as (ii) uncoupling directions where no matching conditions are enforced, see Table 2.

In practice, we need to:

i) complete the average conditions in the coupling directions, by a proper extension so to have pointwise data for the $3 \mathrm{D}$ subproblems;

ii) provide additional scalar pointwise conditions for the 3D subproblems along the uncoupling directions.

To address these tasks, we can follow one of the approaches addressed in Sect. 3.1. All these strategies rely on a weak formulation of the problem that implicitly enforces conditions in the uncoupling directions too. We observe that these new conditions are not coupling conditions anymore and are directly prescribed to the $3 \mathrm{D}$ problem. Thus, either a kinematic or a dynamic condition should be prescribed for each uncoupling directions. For the fluid problem, these additional conditions can be a homogeneous condition for either the tangential velocity

$$
\boldsymbol{u}_{3 D} \cdot \boldsymbol{\tau}=\mathbf{0} \quad \text { at } \Gamma_{f},
$$


or the tangential traction

$$
\boldsymbol{T}_{f}\left(\boldsymbol{u}_{3 D}, p_{3 D}\right) \boldsymbol{n} \cdot \boldsymbol{\tau}=\mathbf{0} \quad \text { at } \Gamma_{f} .
$$

Alternatively, we may force explicitly (and arbitrarily) additional conditions. For instance, we can assume that:

(i) the normal velocity is described by a given velocity profile $\bar{u}$, that is $\boldsymbol{u}_{3 D} \cdot \boldsymbol{n}=F_{3 D}(t) \bar{u}(\boldsymbol{x})$ for a suitable scalar function of time $F_{3 D}(t)$, and the kinematic continuity condition (37) reads

$$
\rho_{f} F_{3 D}(t) \int_{\Gamma_{f}} \bar{u} d \gamma=Q_{1 D}(z=0) ;
$$

(ii) the normal traction is constant over $\Gamma_{f}$, that is from (40) we have

$$
\boldsymbol{T}_{f}\left(\boldsymbol{u}_{3 D}, p_{3 D}\right) \boldsymbol{n} \cdot \boldsymbol{n}=-\theta\left(P_{e x t}(z=0)+\psi\left(A_{1 D}(z=0), A_{0}(z=0), \boldsymbol{\beta}(z=0)\right)\right) ;
$$

(iii) one of the tangential conditions (42),(43) hold.

A similar approach can be used for the structure kinematic condition, by:

(i) postulating a (typically circular) shape for the $3 \mathrm{D}$ vessel interface at the line $\Psi$ equivalently that the radial displacement at $\Psi$ is independent of the circumferential coordinate; in this way, area continuity (38) reduces to

$$
\pi\left(R_{0}+\eta_{3 D}^{r}\left(\Gamma_{s}, r=R_{0}, \varphi\right)\right)^{2}=A_{1 D}(z=0) ;
$$

ii) assuming a constant normal traction on $\Psi$ so that we can derive from (41)

$$
\boldsymbol{T}_{s}\left(\boldsymbol{\eta}_{3 D}\right) \boldsymbol{n} \cdot \boldsymbol{n} d \gamma=-(1-\theta)\left(P_{e x t}(z=0)+\psi\left(A_{1 D}(z=0), A_{0}(z=0), \boldsymbol{\beta}(z=0)\right)\right) \quad \text { at } \Psi
$$

iii) completing the previous conditions at $\Gamma_{s} \backslash \Psi$ along the radial and axial directions, either prescribing null displacements

$$
\eta_{r}=0 \quad \text { and } \quad \boldsymbol{\eta} \cdot \boldsymbol{n}=0 \quad \text { at } \Gamma_{s} \backslash \Psi,
$$

or null tractions

$$
T_{s}^{r}\left(\boldsymbol{\eta}_{3 D}\right)=0 \quad \text { and } \quad \boldsymbol{T}_{s}\left(\boldsymbol{\eta}_{3 D}\right) \boldsymbol{n} \cdot \boldsymbol{n}=0 \quad \text { at } \Gamma_{s} \backslash \Psi
$$

$T_{s}^{r}$ being the radial component of the structure traction.

In general, we can give an abstract formulation to the coupled 3D-1D problem as follows:

$$
\begin{aligned}
& \mathcal{P}_{3 D}\left(\boldsymbol{u}_{3 D}, p_{3 D}, \boldsymbol{\eta}_{3 D} ; \Omega_{1}\right)=0, \\
& \mathcal{K}_{f}\left(\boldsymbol{u}_{3 D}, Q_{1 D}\right)=0, \\
& \mathcal{K}_{s}\left(\boldsymbol{\eta}_{3 D}, A_{1 D}\right)=0, \\
& \mathcal{D}_{f}\left(\boldsymbol{u}_{3 D}, p_{3 D}, Q_{1 D}, A_{1 D}\right)=0, \\
& \mathcal{D}_{s}\left(\boldsymbol{\eta}_{3 D}, A_{1 D}\right)=0, \\
& \mathcal{P}_{1 D}\left(Q_{1 D}, A_{1 D} ;[0, L]\right)=0,
\end{aligned}
$$

where $\mathcal{K}_{f}(\cdot, \cdot)=0$ is the kinematic fluid condition $(37), \mathcal{K}_{s}(\cdot, \cdot)=0$ stems from the structure kinematic conditions in the radial direction $(38), \mathcal{D}_{f}(\cdot, \cdot, \cdot, \cdot)=0$ and $\mathcal{D}_{s}(\cdot, \cdot)=0$ relate to the 
continuity of the fluid and structure tractions in the normal direction (40)-(41), and as usual $\mathcal{P}_{3 D}$ and $\mathcal{P}_{1 D}$ are abstract representations of the stand-alone 3D and 1D problems, respectively. Different interface conditions yield different global problems. The choice of those conditions is therefore critical to guarantee the well posedness (and ultimately the reliability) of the coupled multiscale model. It is therefore important to analyze the property of energy conservation of every approach and its impact on the physical consistency as well as numerical stability.

Remark 6. In the conditions (45)-(46) the area $A_{1 D}$ occurs in both the structure displacement and fluid traction interface conditions, as a consequence of the constitutive law in the $1 D$ subproblem. For the $3 D$ problem this may result in an unphysical constraint between the fluid and the structure. In fact, the material mismatch between the two subproblems - different vessel laws are considered in the $1 D$ and $3 D$ subproblems - may lead to unreliable results in the $3 D$ domain. A practical cure consists of replacing the kinematic interface conditions for the structure (such as (46)) by assuming that condition (47) (or alternatively (48)) holds true also at $\Psi$. Using one of these conditions in fact decouples the $3 D$ structure and the $1 D$ reduced model. In this case, $(49 \mathrm{c})$ reduces to $\mathcal{K}_{s}\left(\boldsymbol{\eta}_{3 D}\right)=0$.

\subsubsection{Energy estimates and interface conditions}

For the analysis of the 3D-1D coupled problem, let us assume that the boundary conditions over the proximal and the distal portions of the boundary of $\bar{\Omega}_{1} \cup[0, L]$ and the external side $\Sigma_{\text {ext }}$ of the $3 \mathrm{D}$ domain are homogeneous, i.e.

$$
\begin{array}{lr}
\boldsymbol{u}_{3 D}=\mathbf{0} & \text { at } \Sigma_{f, \text { prox }}(t), \quad t>0 \\
\widetilde{\boldsymbol{\eta}}_{3 D}=\mathbf{0} & \text { at } \widetilde{\Sigma}_{s, \text { prox }}(t), \quad t>0, \\
\alpha_{S T} \widetilde{\boldsymbol{\eta}}_{3 D}+\widetilde{\boldsymbol{T}}_{s}\left(\widetilde{\boldsymbol{\eta}}_{3 D}\right) \widetilde{\boldsymbol{n}}=\mathbf{0} & \text { at } \widetilde{\Sigma}_{e x t}, \quad t>0
\end{array}
$$

for the 3D subproblem (we recall that $\alpha_{S T}$ is the coefficient accounting for the presence of surrounding tissues introduced in (3)) and

$$
Q_{1 D}=0 \quad \text { at } z=L,
$$

for the $1 \mathrm{D}$ one. Moreover, we assume for simplicity $P_{\text {ext }}=0$.

Let us introduce the following total energy for the $3 \mathrm{D}$ problem $[65,72]$

$$
\mathcal{E}_{3 D}(t)=\frac{\rho_{f}}{2} \int_{\Omega_{f}}\left|\boldsymbol{u}_{3 D}\right|^{2} d \Omega+\int_{\widetilde{\Omega}_{s}} \frac{\rho_{s}}{2}\left|\dot{\overrightarrow{\boldsymbol{\eta}}}_{3 D}\right|^{2} d \Omega+\int_{\widetilde{\Omega}_{s}} \Theta\left(\widetilde{\boldsymbol{\eta}}_{3 D}\right) d \Omega+\int_{\widetilde{\Sigma}_{e x t}} \alpha_{S T}\left|\widetilde{\boldsymbol{\eta}}_{3 D}\right|^{2} d \gamma
$$

and the following one for the 1D model [65]

$$
\mathcal{E}_{1 D}(t)=\frac{\rho_{f}}{2} \int_{0}^{L} A_{1 D} \bar{u}_{1 D}^{2} d x+\int_{0}^{L} \chi\left(A_{1 D}\right) d x
$$

where $\chi(A)=\int_{A_{0}}^{A} \psi(\tau) d \tau, \psi$ being the vessel law (see (6), we outlined its dependence on $A$ only as it is functional to the estimates we are interested in), and $\Theta(\widetilde{\boldsymbol{\eta}})$ is the strain energy density function introduced in Section 2.1. Notice that the stand-alone 3D and 1D problems satisfy bounds for this energy functionals as proved in [65]. 
Let $P_{t o t}=\psi(A)+\frac{\rho_{f}}{2} \bar{u}^{2}$ be the total pressure for the 1D model, and $p_{t o t}$ in (29) the total pressure for the $3 \mathrm{D}$ model. Then, we have the following result that extends the bounds to the multiscale case [72].

Proposition 1. For the interface coupling conditions holding at $\Gamma$ let us assume that the following inequality holds

$$
\int_{\Gamma_{f}} \boldsymbol{T}_{f}\left(\boldsymbol{u}_{3 D}, p_{t o t, 3 D}\right) \boldsymbol{n} \cdot \boldsymbol{u}_{3 D} d \gamma+\int_{\Gamma_{s}} \boldsymbol{T}_{s}\left(\boldsymbol{\eta}_{3 D}\right) \boldsymbol{n} \cdot \dot{\boldsymbol{\eta}}_{3 D} d \gamma+Q_{1 D}(z=0) P_{t o t, 1 D}(z=0) \leq 0 .
$$

Then, the coupled 3D-1D problem (1),(7),(50),(51) satisfies the energy decay property

$$
\frac{d}{d t}\left(\mathcal{E}^{3 D}(t)+\mathcal{E}^{1 D}(t)\right) \leq 0
$$

for all $t>0$.

In particular for inequality (52) to be fulfilled it is sufficient that the interface conditions

$$
\begin{aligned}
& \rho_{f} \int_{\Gamma_{f}} \boldsymbol{u}_{3 D} \cdot \boldsymbol{n} d \gamma=Q_{1 D}(z=0), \\
& \left.\left(\boldsymbol{T}_{f}\left(\boldsymbol{u}_{3 D}, p_{t o t, 3 D}\right) \boldsymbol{n}\right)\right|_{\Gamma_{f}}=-P_{t o t, 1 D}(z=0) \boldsymbol{n}
\end{aligned}
$$

hold for the fluid, together with

$$
\boldsymbol{T}_{s}\left(\boldsymbol{\eta}_{3 D}\right) \boldsymbol{n}=\mathbf{0} \text { at } \Gamma_{s}
$$

for the structure [69, 72]. Similarly, inequality (52) holds if relation (54) is replaced by

$$
\begin{array}{ll}
\boldsymbol{\eta}_{3 D} \cdot \boldsymbol{n}=\mathbf{0} & \text { at } \Gamma_{s}, \\
\left(\boldsymbol{T}_{s}\left(\boldsymbol{\eta}_{3 D}\right) \boldsymbol{n}\right) \times \boldsymbol{n}=\mathbf{0} & \text { at } \Gamma_{s} .
\end{array}
$$

Note that (54) and (55) are in fact independent of the 1D model, resulting in boundary conditions for the $3 \mathrm{D}$ structure problems only. This introduces a discontinuity in the displacement between the 3D and the $1 \mathrm{D}$ model.

Instead, conditions

$$
\frac{1}{\left|\Gamma_{f}\right|} \int_{\Gamma_{f}} \boldsymbol{T}_{f}\left(\boldsymbol{u}_{3 D}, p_{t o t, 3 D}\right) \boldsymbol{n} \cdot \boldsymbol{n} d \gamma=-P_{t o t, 1 D}(z=0),
$$

or

$$
\frac{1}{\left|\Gamma_{f}\right|} \int_{\Gamma_{f}} p_{t o t, 3 D} d \gamma=P_{t o t, 1 D}(z=0)
$$

do not guarantee the fulfillment of (52) and thus the energy decay property.

Also conditions involving the kinetic pressure (as opposed to the total pressure considered in (56)-(57)) like

$$
\frac{1}{\left|\Gamma_{f}\right|} \int_{\Gamma_{f}} \boldsymbol{T}_{f}\left(\boldsymbol{u}_{3 D}, p_{3 D}\right) \boldsymbol{n} \cdot \boldsymbol{n} d \gamma=-\psi\left(A_{1 D}(z=0)\right)
$$


or

$$
\frac{1}{\left|\Gamma_{f}\right|} \int_{\Gamma_{f}} p_{3 D} d \gamma=\psi\left(A_{1 D}(z=0)\right)
$$

do not satisfy condition (52). More precisely, in this case we have

$$
\frac{d}{d t}\left(\mathcal{E}^{3 D}(t)+\mathcal{E}^{1 D}(t)\right)=\frac{\rho_{f}}{2}\left(\frac{\left(Q_{1 D}(t, z=0)\right)^{3}}{\left(A_{1 D}(t, z=0)\right)^{2}}-\int_{\Gamma_{f}}\left|\boldsymbol{u}_{3 D}(t)\right|^{2} \boldsymbol{u}_{3 D}(t) \cdot \boldsymbol{n} d \gamma\right) .
$$

Even though (theoretically) the right hand side is not necessarily (always) negative [72], numerical evidence suggests that these conditions are actually stable, see [130].

Instead of conditions (58) or (59), in analogy with the general treatment of the dynamic conditions provided by (40)-(41), one could also consider a dynamic condition for the structure, obtaining either

$$
\frac{1}{\left|\Gamma_{f}\right|} \int_{\Gamma_{f}} \boldsymbol{T}_{f}\left(\boldsymbol{u}_{3 D}, p_{3 D}\right) \boldsymbol{n} \cdot \boldsymbol{n} d \gamma=-\theta \psi\left(A_{1 D}(z=0)\right)
$$

or

$$
\frac{1}{\left|\Gamma_{f}\right|} \int_{\Gamma_{f}} p_{3 D} d \gamma=\theta \psi\left(A_{1 D}(z=0)\right)
$$

in combination with

$$
\frac{1}{|\Psi|} \int_{\Psi} \boldsymbol{T}_{s}\left(\boldsymbol{\eta}_{3 D}\right) \boldsymbol{n} \cdot \boldsymbol{n} d \gamma=-(1-\theta) \psi\left(A_{1 D}(z=0), A_{0}(z=0), \boldsymbol{\beta}(z=0)\right),
$$

for a suitable $\theta \in[0,1]$.

\subsubsection{Further developments and comments}

Coupling of a $1 D$ model with a $3 D$ rigid model.. The arterial pressure wave propagation involves wave lengths typically larger than the size of a domain to be treated by a full 3D model. This may be troublesome for coupled problems because on the one hand the physical consistency requires to treat fluid-structure interaction in both the subproblems, however the 3D subproblem is affected by a significant computational burden when FSI is included. Depending on the applications, it may be reasonable to still approximate the $3 \mathrm{D}$ model as a rigid one, in particular when the $1 \mathrm{D}$ model is essentially regarded as a physically consistent way of supplying reliable boundary conditions for the $3 \mathrm{D}$ one. However, this approximation generates a strong discontinuity in the way the wall dynamics is described in the overall problem. This may originate spurious reflections and numerical instabilities at the 3D-1D interface.

A possible remedy has been proposed in [157], by pretending the $3 \mathrm{D}$ model to be compliant at the interface with the $1 \mathrm{D}$ submodel by introducing lumped compliances. This approach can be interpreted as a 3D-0D-1D coupling, the 0D being the "virtual" portion of the domain where the effects of compliance are introduced in the model. Alternatively, this modification can be regarded as a modification of the interface conditions between the 3D (only fluid) model and the 1D one to introduce the effects of compliance. Here, we follow this second interpretation. In particular, let us define

$$
P_{3 D}=\frac{1}{\left|\Gamma_{f}\right|} \int_{\Gamma_{f}} p_{3 D} d \gamma, \quad Q_{3 D}=\rho_{f} \int_{\Gamma_{f}} \boldsymbol{u}_{3 D} \cdot \boldsymbol{n} d \gamma
$$


Then, consider the interface conditions

$$
\begin{aligned}
& P_{1 D}=P_{3 D}-L \frac{d Q_{3 D}}{d t}-R_{2} Q_{3 D}+R_{1} C \frac{d P_{3 D}}{d t}-R_{1} C L \frac{d^{2} P_{3 D}}{d t^{2}}-R_{1} Q_{3 D} \\
& Q_{3 D}=Q_{1 D}-R_{1} C \frac{d Q_{1 D}}{d t}-C \frac{d P_{1 D}}{d t}
\end{aligned}
$$

to couple the rigid 3D model to the $1 \mathrm{D}$ problem. Here, $R_{1}, R_{2}$ are two resistances (accounting for viscous effects), $L$ is related to blood inertia and $C$ gathers the compliance of the $3 \mathrm{D}$ model to the interface with the $1 \mathrm{D}$ to damp spurious reflections induced by the structural discontinuity. The cost of the 3D solution is simply that of a fluid problem solved in a domain with rigid boundaries. Should we follow the 3D-0D-1D interpretation of this approach, these conditions stem from simple hydraulic network of $\pi$ type interfacing the two subproblems.

Results presented in [157] show that with an appropriate choice of the parameters $R_{1}, R_{2}, L$ and $C$, flow rate and pressure at the systemic level are acceptable, while keeping relatively low computational costs. Yet, choosing the parameters may be troublesome in patient-specific settings and ad hoc data assimilation procedures are recommended.

The role of the characteristics for the interface conditions.. As we discussed in Sect. 3.2, the 1D problem is strictly hyperbolic and from the mathematical standpoint it features two characteristic or Riemann variables propagating in two opposite directions along the $1 \mathrm{D}$ domain. Because of the hyperbolic nature of the problem, at the interface with the 3D model it is appropriate the prescription of the Riemann variable that is entering $\Omega_{1 D}$. In our example, with the $1 \mathrm{D}$ domain located distally to $\Omega_{3 D}$, this is $W_{1}$. For this reason, one among the pressure or traction interface conditions (53b), (56), (57), (58) or (59) can be replaced in practice by a condition expressing the continuity of $W_{1}$ [65]. In particular, at each time $t$ we may consider condition (53a) and

$$
W_{1}(z=0)=\zeta_{1}\left(\left|\Gamma_{f}\right|, Q_{3 D}\right),
$$

with $\zeta_{1}$ given by (32) and $Q_{3 D}$ by (62).

It is possible to prove that this condition together with (53a) implies the continuity of the area at the interface while the continuity of the pressure/traction is not guaranteed.

\subsection{D-OD coupling}

\subsubsection{Formulation of the problem}

In some cases the knowledge of the space dependence of the variables of interest is not crucial in the downstream region $\Omega_{2}$. The dynamics here can be described by a compartment represented by a lumped parameter model. Following up the outline of the present section, this case can be regarded as a further simplification of the 3D-1D model previously considered, where the $1 \mathrm{D}$ downstream subdomain is replaced by a 0D description.

Interface conditions between the 3D and the $0 \mathrm{D}$ subdomain can be devised by proceeding as done for the 3D-1D case, in particular by prescribing again kinematic and dynamic continuity conditions, in a form that is adapted to the context of the lumped parameter model. For instance, if $\mathcal{P}_{3 D}\left(\boldsymbol{u}_{3 D}, p_{3 D}, \boldsymbol{\eta}_{3 D} ; \Omega_{1}\right)=0$ is the abridged notation for the $3 \mathrm{D}$ model (including the inlet conditions on fluid velocity or traction and on structure displacement or traction) and $\mathcal{P}_{0 D}\left(Q_{0 D}, P_{0 D}\right)=0$ represents the downstream lumped parameter model (where we highlighted the 
dependence on the proximal quantities $Q_{0 D}$ and $P_{0 D}$ ), we can devise a set of interface conditions reading as

$$
\begin{gathered}
\rho_{f} \int_{\Gamma_{f}} \boldsymbol{u}_{3 D} \cdot \boldsymbol{n} d \gamma=Q_{0 D}, \\
\frac{1}{\left|\Gamma_{f}\right|} \int_{\Gamma_{f}} \boldsymbol{T}_{f}\left(\boldsymbol{u}_{3 D}, p_{3 D}\right) \boldsymbol{n} \cdot \boldsymbol{n} d \gamma=-\theta P_{0 D}, \\
\int_{0}^{2 \pi} \int_{0}^{R_{0}+\eta_{3 D}^{r}\left(\Gamma_{s}, r=R_{0}, \varphi\right)} r d r d \varphi=\psi^{-1}\left(P_{0 D}\right), \\
\frac{1}{|\Psi|} \int_{\Psi} \boldsymbol{T}_{s}\left(\boldsymbol{\eta}_{3 D}\right) \boldsymbol{n} \cdot \boldsymbol{n} d \gamma=-(1-\theta) P_{0 D},
\end{gathered}
$$

where $\psi$ is given by the the algebraic equation (11) relating the area and the pressure and used in the derivation of the $0 \mathrm{D}$ model and where again $\theta \in[0,1]$. In analogy with the 3D-1D case, we can use for the dynamic fluid continuity a condition like (59) on the mean pressure instead of the normal traction. As for the coupling between $3 \mathrm{D}$ and $1 \mathrm{D}$ models, also in this case the conditions are "defective" for the 3D subdomain and the problem needs to be completed as done in the previous section.

When coupling 3D with 0D models, three issues need to be emphasized:

1. In the derivation of the $0 \mathrm{D}$ model one assumption is that convective terms are in general small. For this reason, the basic derivation for an arterial segment leads to a linear model, the nonlinear terms having been dropped. Consequently, on the 0D side the pressure coincides with the total pressure, so that a total pressure interface condition simply reads

$$
\frac{1}{\left|\Gamma_{f}\right|} \int_{\Gamma_{f}} p_{t o t, 3 D} d \gamma=P_{0 D} .
$$

2. As pointed out in Sect. 2.3, the mathematical formulation of the 0D model does not separate the equations of the dynamics from the "boundary conditions"; the latter are directly incorporated in the system of ordinary differential equations, since the space dependence in the model is dropped. This feature is crucial when devising segregated algorithms for solving the 3D-0D problem, since the mathematical representation of the 0D problem has to be compatible with the associated interface conditions. We will address this concept in Section 5 .

3. As for the 3D-1D coupling, the mismatch of the structural constitutive laws separately postulated in the two subdomains may lead to some spurious effects. In this case, often the continuity of the displacement and tractions for the structure problem (65c)-(65d) are dropped and they are replaced by a homogeneous boundary condition for $\boldsymbol{\eta}_{3 D}$ or for $\boldsymbol{T}_{\boldsymbol{s}}\left(\boldsymbol{\eta}_{3 D}\right)$.

In many applications $[219,113]$ when coupling 3D and 0D models, the lumped parameter subdomain is intended to provide just a transfer function to incorporate the presence of the peripheral 
impedance into the simulation of the 3D subdomain. In this case, the windkessel model and its variants addressed in Sect. 2.3.2 provide an excellent simplified description to be embedded straightforwardly into the solution of the 3D problem. We illustrate a semi-analytic approach for solving this case in the next subsection.

More generally, coupling between the two models stems genuinely from the necessity of solving the different scales (local and global) and a windkessel model is not accurate enough for the level of detail required to the systemic submodel. An example extensively addressed in the literature is given by the numerical modeling of the Total CavoPulmonary Connection (TCPC), see e.g. $[119,118,136,93]$. In this case, the 0D model - that is in general nonlinear due to the presence of the valves - is not directly included in the 3D solution; segregated approaches are indeed more indicated. This topic will be covered in Section 5 .

\subsubsection{Monolithic Solution of 3D-OD windkessel models}

When the 0D differential system can be formally solved by standard techniques for ordinary differential equations, the resulting solutions combine pressure and flow rate to provide a Robintype defective boundary condition for the 3D fluid problem.

We illustrate this outcome in the case of the 3-element windkessel peripheral models. The extension to the 4-element windkessel models is technically more involved but promptly derived following similar steps.

Let us consider the 3-element windkessel model represented in Fig. 6(b). Referring to the notation reported in the figure, we use here the subscript " $0 \mathrm{D}$ " to denote quantities related to the lumped parameter model. This model corresponds to the differential equation

$$
\frac{d\left(P_{0 D}-R_{1} Q_{0 D}\right)}{d t}+\frac{1}{R_{2} C}\left(P_{0 D}-R_{1} Q_{0 D}\right)=\frac{Q_{0 D}}{C} .
$$

By a standard application of the method of integrating factors (see e.g. [40]) we find

$$
P_{0 D}(t)=R_{1} Q_{0 D}(t)+\left(P_{0 D}(0)-R_{1} Q_{0 D}(0)\right) e^{-t /\left(R_{2} C\right)}+\frac{1}{C} \int_{0}^{t} e^{\tau-t /\left(R_{2} C\right)} Q_{0 D}(\tau) d \tau .
$$

For the continuity of the normal velocity and of the tractions that we postulate at the interface, we set $Q_{0 D}=\rho_{f} \int_{\Gamma_{f}} \boldsymbol{u}_{3 D} \cdot \boldsymbol{n} d \gamma$, and $P_{0 D}=-\frac{1}{\left|\Gamma_{f}\right|} \int_{\Gamma_{f}} \boldsymbol{T}_{f}\left(\boldsymbol{u}_{3 D}, p_{3 D}\right) \boldsymbol{n} \cdot \boldsymbol{n} d \gamma$. Once plugged in (66), these lead to the following resistance defective condition

$$
\begin{array}{r}
\frac{1}{\left|\Gamma_{f}\right|} \int_{\Gamma_{f}} \boldsymbol{T}_{f}\left(\boldsymbol{u}_{3 D}, p_{3 D}\right) \boldsymbol{n} \cdot \boldsymbol{n} d \gamma+R_{1} \rho_{f} \int_{\Gamma_{f}} \boldsymbol{u}_{3 D} \cdot \boldsymbol{n} d \gamma+\frac{\rho_{f}}{C} \int_{0}^{t} e^{\tau-t /\left(R_{2} C\right)} \int_{\Gamma_{f}} \boldsymbol{u}_{3 D}(\boldsymbol{x}, \tau) \cdot \boldsymbol{n} d \gamma d \tau= \\
\quad\left(\frac{1}{\left|\Gamma_{f}\right|} \int_{\Gamma_{f}} \boldsymbol{T}_{f}\left(\boldsymbol{u}_{3 D}(\boldsymbol{x}, 0), p_{3 D}(\boldsymbol{x}, 0)\right) \boldsymbol{n} \cdot \boldsymbol{n} d \gamma+R_{1} \rho_{f} \int_{\Gamma_{f}} \boldsymbol{u}_{3 D}(\boldsymbol{x}, 0) \cdot \boldsymbol{n} d \gamma\right) e^{-t /\left(R_{2} C\right)} \boldsymbol{n} .
\end{array}
$$

The previous condition can eventually be incorporated into the variational formulation of the Navier-Stokes equations [219]. 
In practice, in a time-discrete setting for the numerical solution of the problem, (66) is applied over the timeline of the single time step between $t^{n}$ and $t^{n+1}$, used in combination with a quadrature rule (e.g. the trapezoidal rule) to treat the third term at the left hand side, leading to

$$
\frac{1}{\left|\Gamma_{f}\right|} \int_{\Gamma_{f}} \boldsymbol{T}_{f}\left(\boldsymbol{u}_{3 D}^{n+1}, p_{3 D}^{n+1}\right) \boldsymbol{n} \cdot \boldsymbol{n} d \gamma+\rho_{f}\left(R_{1}+1 / 2 C\right) \int_{\Gamma_{f}} \boldsymbol{u}_{3 D}^{n+1} \cdot \boldsymbol{n} d \gamma=\mathcal{G}\left(\boldsymbol{u}_{3 D}^{n}, p_{3 D}^{n}, \boldsymbol{u}_{3 D}^{0}, p_{3 D}^{0}\right),
$$

for a suitable function $\mathcal{G}$ of the outlined arguments.

Remark 7. For the practical prescription of condition (67) a possible solution is given by evaluating the flow rate term through a suitable extrapolation from previous time steps, thus leading to a mean traction condition, which could be prescribed, e.g., with the do-nothing approach presented in Sect 3.1.2. This solution is very attractive when one wants to use a commercial solver. In principle, we could follow a dual approach where we extrapolate the mean pressure from previous time steps, thus obtaining in fact a flow rate condition. Although possible, this approach requires the treatment of defective flux conditions. As we have seen in Sect. 3, this is much more complicated, so the previous approach is by far the most popular.

\subsection{D-OD coupling}

At last, we consider the case when a lumped parameter model is coupled to a 1D model in order to include the effects of systemic circulation, for instance when peripheral circulation is accounted for by means of a suitable boundary condition to close a $1 \mathrm{D}$ model. In this case, the role of the lumped parameter model is to calculate the pressure wave reflections generated by peripheral districts and by the microcirculation [70]. In a different context, 0D models have been coupled with a 1D description of the circulatory network in [68] to include the action of the heart.

Coherently with the previous assumptions in the present section, we consider here the case when a single arterial cylindrical segment is described by a $1 \mathrm{D}$ model, proximally in a region $\Omega_{1}$, and by a $0 \mathrm{D}$ model in a region $\Omega_{2}$ located distally. As done in Sect. 4.1, we start with a split-bysubdomain representation of the same problem in terms of $1 \mathrm{D}$ models. Over the segment spanned by the axial abscissa $z \in[-L, L]$ the unsplit $1 \mathrm{D}$ problem

$$
\mathcal{P}_{1 D}\left(Q_{1 D}, A_{1 D},[-L, L]\right)=0
$$

is formulated as the coupling of two $1 \mathrm{D}$ subproblems in $\Omega_{1} \equiv[-L, 0]$ and $\Omega_{2} \equiv[0, L]$, respectively

$$
\begin{aligned}
& \mathcal{P}_{1 D}\left(Q_{1 D}^{1}, A_{1 D}^{1},[-L, 0]\right)=0, \\
& Q_{1 D}^{1}(z=0)=Q_{1 D}^{2}(z=0), \\
& \psi\left(A_{1 D}^{1}(z=0)\right)=\psi\left(A_{1 D}^{2}(z=0)\right) \\
& \mathcal{P}_{1 D}\left(Q_{1 D}^{2}, A_{1 D}^{2},[0, L]\right)=0 .
\end{aligned}
$$

Here we have understood convenient conditions at the proximal $z=-L$ and distal $z=L$ boundaries in the abridged notation $P_{1 D}\left(Q_{1 D}^{i}, A_{1 D}^{i}, \cdot\right)$ for $i=1,2$. In addition, we introduced interface conditions based on the continuity of mass and momentum. The latter is written in terms of pressure, regarded as function of the area $A_{1 D}^{i}, i=1,2$. Other interface conditions can be considered as well, for instance involving the total pressure rather than the pressure. If the function 
$\psi$ representing the vessel law is the same for the two subproblems, as it is often the case, the continuity of the pressure and that of the flow rates implies the continuity of the total pressure. From the mathematical point of view it makes sense to consider alternative equivalent interface conditions formulated in terms of characteristic variables $W_{i}^{j}\left(A_{1 D}^{i}, Q_{1 D}^{i}\right), i, j=1,2$, since they precisely consider quantities with a well defined direction of propagation.

Next, we replace the downstream 1D problem with a suitable 0D model for a cylindrical segment (and including the appropriate boundary conditions at $z=L$ ), leading to the geometric multiscale model

$$
\begin{aligned}
& \mathcal{P}_{1 D}\left(Q_{1 D}, A_{1 D} ;[-L, 0]\right)=0, \\
& Q_{1 D}(z=0)=Q_{0 D}, \\
& \psi\left(A_{1 D}(z=0)\right)=P_{0 D}, \\
& \mathcal{P}_{0 D}\left(Q_{0 D}, P_{0 D}\right)=0,
\end{aligned}
$$

where again $Q_{0 D}$ and $P_{0 D}$ represent the proximal quantities in the $0 \mathrm{D}$ model. Also in this case, we can replace one of the interface conditions stemming from the continuity of mass and momentum with one for the characteristic variable $W_{2}$

$$
W_{2}(z=0)=\zeta_{2}\left(\psi^{-1}\left(P_{0 D}\right), Q_{0 D}\right),
$$

with $\zeta_{2}$ given by (32). This prevents the occurrence of numerical artifacts introduced by approximation errors that trigger unphysical reflections at $z=L[70,63]$.

In comparison with the 3D-0D case, this coupling is much more intuitive, since both $1 \mathrm{D}$ and 0D models compute average area and flow rate over different points of the axis, and no defective conditions need to be sorted out at the coupling interface. In particular, if the $0 \mathrm{D}$ model is simple (like for a cylindrical segment as well as a windkessel model) a semi-analytical approach based on the method of integrating factors illustrated in the previous section can be pursued. This means that the pressure $P_{0 D}$ can be explicitly computed as a function of the flow rate $Q_{0 D}$ and used straightforwardly in the prescription of $W_{2}$.

More in general, when the 0D network is more complicated for the presence of several compartments and/or nonlinear terms, a partitioned approach has to be preferred, as we will see in the next Section.

\section{Numerical strategies}

The numerical solution of the coupled geometric multiscale problems presented in Sect. 4 requires special care. The intrinsic heterogeneity and the diverse nature of the problem components drives the set up of a solution algorithm and its analysis. Generally speaking, two basic options can be pursued, a monolithic approach and a partitioned or segregated one. The latter strategy seems to be more natural - apart from special cases like the 3D-windkessel coupled model addressed in Sect. 4.2.2 - and is discussed in the next subsections.

As for the temporal stability of the solver, this generally follows from the restrictions induced by the time advancing schemes adopted for the different components of the multiscale model. While implicit or semi-implicit approaches are preferred for the 3D models to avoid stability restrictions of parabolic type in the fluid problem (e.g. the time step $\Delta t$ bounded by a quantity scaling with $\left.h^{2}\right), 1 \mathrm{D}$ models are usually solved by explicit methods subject to CFL stability conditions. General 
statements on the most restrictive conditions are difficult to draw, as they depend on the specific problem (the vascular district for the 3D model as well as the portion of the network for the 1D one). For this reason, in partitioned schemes where the different solvers are called in an either sequential or parallel fashion, it is worth resorting to multi-level time stepping techniques, where the different geometric components are solved with individual convenient time steps and matching interface conditions are fulfilled thanks to suitable synchronization procedures [31, 131] - see also Sect. 6.4.2

In the remainder of this Section we keep considering the time discretization of the coupled problems already introduced in Sect. 4. However, we will drop the time index $n+1$ for the sake of notation.

\subsection{Partitioned algorithms: generalities}

A quite natural approach for solving heterogeneous problems is to split them into their homogeneous components and to delegate iterative schemes to enforce their matching. This holds for many applications in multi-physics, and remarkably for fluid-structure interaction problems [178]. Dimensionally heterogeneous problems like the ones generated by the geometric multiscale modeling are no exception. As a matter of fact, the intrinsic modularity of this approach perfectly fits into the need of solving diverse mathematical (and numerical) coupled problems, including 3D, 1D partial as well as ordinary differential equations [70].

In the previous section, we introduced the sequence (i) domain splitting; (ii) model simplification, to obtain in a systematic way geometric multiscale models. Here we bring this procedure to the final step, namely the iterative substructuring formulation [178]. This introduces a sequence of dimensionally homogeneous problems in the two subdomains, where the interface conditions iteratively provide boundary data.

When resorting to these domain decomposition techniques, there are basically two fundamental issues to consider at a very general level, namely (a) the well posedness of each subproblem at every iteration; (b) the convergence of the iterative procedure.

Point (a) in general may be standard when domain decomposition is used as a framework to exploit parallel architectures for solving homogeneous problems. This, however, is not the case of geometric multiscale models, where the dimensional mismatch unavoidably leads to defective boundary problems for the 3D model. The well posedness of the 3D FSI model - at both the continuous and then at the numerical level - with defective boundary data needs to be carefully analyzed - see Sect. 3.1.

Another non-trivial issue arises when considering lumped parameter models for which - as previously pointed out - the concept of boundary data is SOMEHOW INAPPROPRIATE SINCE OD MODELS DO NOT RETAIN ANY EXPLICIT SPACE DEPENDENCE, YET INTUITIVE SINCE THEY REPRESENT REGIONS WITH a PRECISE SPATIAL AND FUNCTIONAL LOCATION. We will address these issues in detail in the next subsections.

As for point (b), we can take advantage of standard techniques for iterative substructuring methods, including either sequential block Gauss-Seidel like or parallel block Jacobi like formulations of the algorithm. While it is fairly complicated to apply the general theory of iterative schemes to our dimensionally heterogeneous problems, the gallery of examples in Sect. 6 illustrates successful schemes for different possible couplings.

It is worth noting that, originally conceived as a numerical solution procedure, the partitioned formulation turned out to be an effective tool to investigate well posedness of geometric multiscale problems, by applying appropriate fixed point theorems, as we will specify in Sect. 5.3 [181, 63]. 
Our exemplifications here will be limited to the simple cylindrical domain introduced in Sect. 4. For the sake of clarity, we summarize here the notation. With a little abuse we will denote the time-discrete stand-alone models with the same compact notation used in Section 4 for their time-continuous counterparts. Hence:

- $\mathcal{P}_{3 D}\left(\boldsymbol{u}_{3 D}, p_{3 D}, \boldsymbol{\eta}_{3 D} ; \Omega\right)=0$ represents the time-discrete 3D problem in the FSI domain $\Omega$ with suitable boundary conditions at the fluid and structure inlets and at the physical boundary. The specific time-discretization scheme used is not important at this stage. At the interface with other subdomains we postulate conditions like the ones specified in Sect. 4 that in the framework of partitioned algorithms will provide boundary data.

- $\mathcal{P}_{1 D}\left(Q_{1 D}, A_{1 D} ;[0, L]\right)=0$ represents a time-discrete $1 \mathrm{D}$ model in the domain $[0, L]$. On one side of the domain we will have standard boundary conditions inherited from the boundaryvalue problem; on the other side, interface conditions with the corresponding subdomain will provide the necessary data to solve it as a standard stand-alone problem.

- $\mathcal{P}_{0 D}\left(S_{0 D}\right)=0$ represents one of the possible problems originating by the time discretization of the ordinary differential system associated with the networks depicted in Fig. 5, where $S_{0 D}$ is the proximal variable (pressure or flow rate) that determines the state of the network.

\subsection{The $3 D-1 D$ case}

Let us consider a partitioned algorithm for the 3D-1D coupled problem. We specifically refer to the time discretization of (49) when interface condition $\mathcal{D}_{f}(\cdot, \cdot, \cdot, \cdot)=0$ is given by (59), as a special instance of (49d). In Algorithm 1 we reported the pseudo-code of this "Flow rate/Mean pressure" scheme where at each iteration the flow rate computed by the $1 \mathrm{D}$ model is used to feed the 3D problem as boundary condition for the fluid, whereas the interface mean pressure computed by the 3D problem provides the boundary condition at the interface with the $1 \mathrm{D}$ model [65]. For the sake of exposition we set $P_{e x t}=0$ and we do not report explicitly the dependence of $A_{1 D}$ on $A_{0}$ and $\boldsymbol{\beta}$.

In Algorithm 1, the kinematic structure condition (49c) has been rewritten in order for the 1D area at the previous iteration to feed the $3 \mathrm{D}$ problem.

Any of the methods illustrated in Section 3.1 can be used to solve the defective 3D problems and compute numerically the 3D fluid velocity and pressure as well as the structure displacement.

As an alternative to Algorithm 1, one could swap the roles of the interface conditions, prescribing the mean pressure to the $3 \mathrm{D}$ problem and the flow rate to the $1 \mathrm{D}$ problem ("Mean pressure/Flow rate" algorithm). In this case at each iteration we solve a mean pressure defective condition for the 3D problem, with one of the strategies described in Section 3.1. The two schemes, "Flow rate/Mean pressure" and "Mean pressure/Flow rate", converge to the same solution and the choice between them is essentially driven by efficiency arguments.

However, there are other possible conditions to be considered for the coupling. For instance, interface condition (57) involving the mean total pressure can replace (59) in Algorithm 1. In this case, the following alternative boundary condition on the mean pressure for the $1 \mathrm{D}$ problem replaces (73b) accordingly

$$
P_{1 D}^{(k)}(z=0)=\frac{1}{\left|\Gamma_{f}\right|} \int_{\Gamma_{f}} p_{3 D}^{(k)} d \gamma+\frac{1}{2}\left(\frac{1}{\left|\Gamma_{f}\right|} \int_{\Gamma_{f}}\left|\boldsymbol{u}_{3 D}^{(k-1)}\right|^{2} d \gamma-\left(\frac{Q_{1 D}^{(k-1)}(z=0)}{\rho_{f} A_{1 D}^{(k-1)}(z=0)}\right)^{2}\right) .
$$


Algorithm 1 "Flow rate/Mean pressure" scheme for the 3D-1D coupled problem (49) with $\mathcal{D}_{f}(\cdot, \cdot, \cdot, \cdot)=0$ given by $(59)$

Given the quantities at previous time steps and at the previous iteration $k-1$, a tolerance $\varepsilon$, and $\omega \in(0,1]$, and setting $Q_{1 D}^{(0)}(z=0)=Q_{1 D}^{n}(z=0)$,

WHILE

$$
\left|Q_{1 D}^{(k-1)}\right|_{z=0}-\left.Q_{1 D}^{(k-2)}\right|_{z=0} \mid \geq \varepsilon
$$

DO // iteration $k$

1. SOLVE the 3D problem

$$
\begin{aligned}
& \mathcal{P}_{3 D}\left(\boldsymbol{u}_{3 D}^{(k)}, p_{3 D}^{(k)}, \boldsymbol{\eta}_{3 D}^{(k)} ; \Omega_{1}\right)=0 \\
& \mathcal{K}_{s}\left(\boldsymbol{\eta}_{3 D}^{(k)}\right)=g\left(A_{1 D}^{(k-1)}(z=0)\right), \\
& \int_{\Gamma_{f}} \boldsymbol{u}_{3 D}^{(k)} \cdot \boldsymbol{n} d \gamma=Q_{1 D}^{(k-1)}(z=0)
\end{aligned}
$$

2. SOLVE the 1D problem

$$
\begin{aligned}
& \mathcal{P}_{1 D}\left(\widetilde{Q}_{1 D}^{(k)}, A_{1 D}^{(k)} ;[0, L]\right)=0 \\
& A_{1 D}^{(k)}(z=0)=\psi^{-1}\left(\frac{1}{\left|\Gamma_{f}\right|} \int_{\Gamma_{f}} p_{3 D}^{(k)} d \gamma\right)
\end{aligned}
$$

3. Relax: $Q_{1 D}^{(k)}=\omega \widetilde{Q}_{1 D}^{(k)}+(1-\omega) Q_{1 D}^{(k-1)}$;

4. Update: $k \rightarrow k+1$.

\section{END DO}


This follows from a proper linearization of (57) with a fixed point argument within the same loop used to enforce the fulfillment of the interface conditions [72].

The same conditions can be enforced differently thanks to identity (30) in the weak formulation of the Navier-Stokes equations and a do-nothing approach as proposed in [69]. In fact, this allows to impose the total pressure as a natural condition, thus avoiding subiterations. Since the interface conditions involve the total pressure, henceforth differing from the ones considered in Algorithm 1 , the converged solution found will be in general different.

Similar considerations hold for the case where the dynamic fluid condition (49d) is provided by the mean traction or mean total traction (given by (58) and (56), respectively), rather than the mean pressure or mean total pressure.

Another scenario is obtained in the "Mean pressure/Flow rate" algorithm when one wants to use conditions (60)-(61) instead of (59). In this case, the 3D problem is equipped with the following boundary conditions at the interface

$$
\begin{aligned}
& \frac{1}{\left|\Gamma_{f}\right|} \int_{\Gamma_{f}} p_{3 D}^{(k)} d \gamma=\theta \psi\left(A_{1 D}^{(k-1)}(z=0)\right), \\
& \frac{1}{|\Psi|} \int_{\Psi} \boldsymbol{T}_{s}\left(\boldsymbol{\eta}_{3 D}^{(k)}\right) \boldsymbol{n} \cdot \boldsymbol{n} d \gamma=-(1-\theta) \psi\left(A_{1 D}^{(k-1)}(z=0)\right) .
\end{aligned}
$$

An alternative approach makes use of the characteristic variables as interface coupling conditions. In particular, in [65] it has been proposed to consider the coupling interface conditions (53a)-(64) and to replace in Algorithm 1 the boundary condition for the 1D model (73b) with the following one:

$$
W_{1,1 D}^{(k)}(z=0)=\zeta_{1}\left(\left|\Gamma_{f}^{(k)}\right|, \rho_{f} \int_{\Gamma_{f}^{(k)}} \boldsymbol{u}_{3 D}^{(k)} \cdot \boldsymbol{n} d \gamma\right) .
$$

In [156], interface conditions based on both the characteristic variables are considered. When a partitioned algorithm is introduced, this leads to a non-linear condition for the 3D fluid given by a combination of mean pressure and flow rate, which could be prescribed again by an iterative method.

An inexact implementation of Algorithm 1 (as well as of all the other partitioned procedures) would consist in performing just one iteration at each time step, yielding an explicit partitioned algorithm, see for example $[69,156]$.

In Algorithm 1 the area at the interface for the 1D model is retrieved by the 3D pressure. Notice however that the area computed in this way is used also as a (defective) condition for the displacement of the 3D structure, following the idea proposed in [65] and applied in [130].

A different class of partitioned algorithms for the 3D-1D coupling has been described in [29], where a block Gauss-Seidel strategy has been applied to the monolithic system obtained after Lagrange multipliers are introduced to enforce the interface continuity conditions (see Section 4.1.3).

\subsection{The $3 D-0 D$ case}

The most intriguing aspect when performing segregated coupling of 3D and lumped parameter models is that the latter does not have an explicit space dependence whilst the coupling with the $3 \mathrm{D}$ subdomain occurs at a specific space location. As we have pointed out in Sect. 2.3.1, 
the key observation here is that the lumped parameter model represents not only the result of a simplification of the $3 \mathrm{D}$ equations, but also of the associated boundary conditions.

For a model retaining space dependence (either 3D or 1D), different data can be prescribed at the boundary. Conversely, the specific formulation of a lumped parameter model of a cylindrical compliant pipe depends on the data prescribed to force its dynamics (see Fig. 5). The portion of the lumped parameter model receiving this input reflects the nature of the data. This leads to the concept of bridging region [181].

The bridging region is an abstract concept to identify the transition between the region occupied by the 3D model and the "region" where the 0D model holds. In fact, it is functionally surrogated by an ODE that makes this transition mathematically correct. The word "correct" refers to the compatibility between the interface conditions that in the segregated algorithm are eventually associated with the subdomains and the surrogate model that governs the transition in the 0D region.

For instance, pressure input data for the $0 \mathrm{D}$ model will require a bridging region for the computation of the flow rates. This bridging region in fact interacts with the 3D model through an inductive term, representing a differential equation for the flow rate. Similarly, flow rate input data call for the pressure at the interface as state variable. This means that the bridging region, mathematically represented by a differential equation for the pressure, will be represented by a compliance term (see Fig. 12).

On the contrary, if we prescribe a pressure (or a flow rate) to feed a compliant (or inertial) region, the dynamic of the pressure (flow rate) there does not need to be computed; the mathematical model of the hydraulic network features a differential equation for pressure (flow rate) and it is thus redundant. It may be conveniently reduced (by eliminating the redundant component) to solve it.

More in general, we say that we have the bridging region compatibility if the data that feed the lumped parameter model are consistent, in the sense that they generate no redundancy in the hydraulic network.

This connection between input data and network topology has a central role when devising partitioned algorithms. In fact, when we segregate the computation of the different subdomains, the interface conditions are iteratively associated with the subproblems as boundary data. In nonoverlapping partitions, we have different conditions (Dirichlet/Neumann, Robin/Robin, etc.) to associate with the different subdomains. For dimensionally heterogeneous models involving a 0D subdomain, the selected boundary condition acts as forcing term for the lumped parameter system. Under bridging region compatibility the nature of this forcing term dictates then the topology of the network. We illustrate this concept in Fig. 12. If we associate flow rate conditions to the $0 \mathrm{D}$ system then the compatible bridging region will have a compliance term (capacitance) at the inlet. Similarly, bridging region compatibility implies that pressure input finds an inertial term (inductance) at the "entrance" of the 0D subdomain. Algorithm 2 illustrates a simple instance of this scenario. More precisely, we consider the coupled problem 3D-0D with interface conditions (65). In this case, the flow rate condition (65a) requires a compatible bridging region like a $\mathcal{L}$ or $\pi$ network, that links the 3D with a compliance term. On the 3D side, the data retrieved iteratively from the lumped parameter model are clearly defective. The mean pressure 3D for the fluid may be completed with the "do-nothing" approach converting pressure data to natural Neumann conditions on the tractions. In fact, the pressure and the area are related by an algebraic law, so that once one is computed the other is promptly recovered. In particular, area retrieved from the lumped parameter model may be used to prescribe a defective structure displacement for 


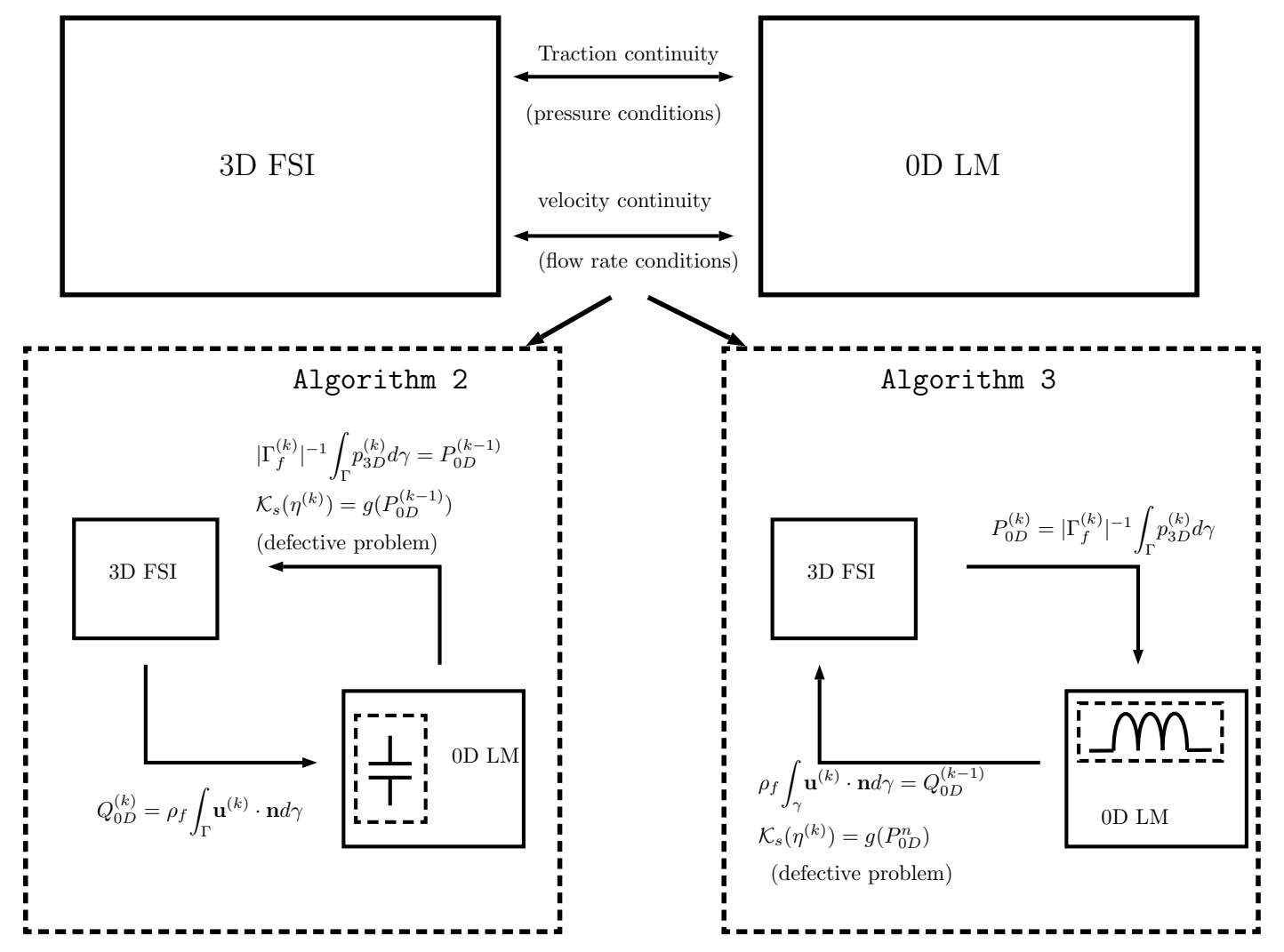

Figure 12: Scheme of the 3D-0D coupling. Depending on the partition of the scheme (either Algorithm 2 or Algorithm 3) the $0 \mathrm{D}$ scheme receives data on flow rate (Algorithm 2) or pressure (Algorithm 3). The interface bridging region needs to be compatible to avoid redundancy in the lumped parameter model: in the former case the compatible bridging region will contain a capacitance described by a differential equation for the pressure; in the latter case the compatible bridging region will contain an inductance described by a differential equation for the flow rate. The 3D problem will be defective and it will be properly solved. 
the 3D problem (see Sect. 3.1.4). In an oversimplified setting, displacement may be simply set to zero, as we discussed in the Remark 6.

In Algorithm 2 we use a strongly or implicit coupling iterative procedure. The different problems are iteratively solved within each time step up to the fulfillment of the chosen convergence criterion. The mean pressure is prescribed to the $3 \mathrm{D}$ model as a mean pressure condition. Again, a condition on the mean normal traction or on the mean total pressure could be considered as well. As for the 3D-1D coupling, an explicit variant performs just one iteration per time step. This is based on a time extrapolation argument of interface variables. For instance, in Algorithm 2 we may perform at each time step $t^{n+1}$ just one iteration by using as initial guess the pressure retrieved at time $t^{n}$ as boundary condition for the $3 \mathrm{D}$ fluid problem. The rationale is that $P_{0 D}^{n}$ is a first order approximation of $P_{0 D}^{n+1}$ with respect to $\Delta t$. This time extrapolation (or similar with higher order dependence on the time step) generally introduces a stability limitation to the time step that may adversely impact the computational advantage of not solving the subproblems several times at each time step - see [175]. We call this second approach weakly or loosely coupled.

\section{Algorithm 2 "Mean pressure/Flow rate" scheme for the 3D-0D coupled problem}

Given the quantities at previous time steps and at the previous iteration $k-1$, a tolerance $\varepsilon$, and $\omega \in(0,1]$, and setting $P_{0 D}^{(0)}=P_{0 D}^{n}$,

\section{WHILE}

$$
\left|P_{0 D}^{(k-1)}-P_{0 D}^{(k-2)}\right| \geq \varepsilon,
$$

DO // iteration $k$

1. SOLVE the 3D problem

$$
\begin{aligned}
& \mathcal{P}_{3 D}\left(\boldsymbol{u}_{3 D}^{(k)}, p_{3 D}^{(k)}, \boldsymbol{\eta}_{3 D}^{(k)} ; \Omega_{1}\right)=0 \\
& \mathcal{K}_{s}\left(\boldsymbol{\eta}_{3 D}^{(k)}\right)=g\left(P_{0 D}^{(k-1)}\right) \\
& \frac{1}{\left|\Gamma_{f}^{k}\right|} \int_{\Gamma_{f}} p_{3 D}^{(k)} d \gamma=P_{0 D}^{(k-1)}
\end{aligned}
$$

2. SOLVE the OD problem

$$
\begin{aligned}
& \mathcal{P}_{0 D}\left(\widetilde{P}_{0 D}^{(k)}\right)=0, \\
& Q_{0 D}^{(k)}=\int_{\Gamma_{f}} \boldsymbol{u}_{3 D}^{(k)} \cdot \boldsymbol{n} d \gamma
\end{aligned}
$$

3. Relaxation: $P_{0 D}^{(k)}=\omega \widetilde{P}_{0 D}^{(k)}+(1-\omega) P_{0 D}^{(k-1)}$;

4. Update: $k \rightarrow k+1$.

\section{END DO}

In Algorithm 3 we illustrate the "dual" case where pressure data are prescribed to the 0D model interfacing with an inductive bridging region, like the one for instance of a $\mathcal{L}$-inverted or $\mathcal{T}$ network (see Fig. 5). Correspondingly, the 3D fluid model receives flow rate prescribed for instance by a Lagrange multiplier approach (see Sect. 3.1). Other approaches for this are possible 
as well. As for the 3D structure, area condition are prescribed by properly extrapolating data from the previous iterations/time steps. Again, simplified coupling is often performed by prescribing null displacement in the axial direction and null traction in the tangential directions.

An example of application of Algorithm 3 (for a 3D problem with rigid boundary) is reported in [211]. $\overline{\text { Algorithm } 3 \text { "Flow rate/Mean pressure" scheme for the 3D-0D coupled problem }}$
Given the quantities at previous time steps and at the previous iteration $k-1$, a tolerance $\varepsilon$, and
$\omega \in(0,1]$, and setting $Q_{0 D}^{(0)}=Q_{0 D}^{n}$,

\section{WHILE}

$$
\left|Q_{0 D}^{(k-1)}-Q_{0 D}^{(k-2)}\right| \geq \varepsilon
$$

DO // iteration $k$ :

1. SOLVE 3D problem

$$
\begin{aligned}
& \mathcal{P}_{3 D}\left(\boldsymbol{u}_{3 D}^{(k)}, p_{3 D}^{(k)}, \boldsymbol{\eta}_{3 D}^{(k)} ; \Omega_{1}\right)=0, \\
& \mathcal{K}_{s}\left(\boldsymbol{\eta}_{3 D}^{(k)}\right)=g\left(P_{0 D}^{(k-1)}\right), \\
& \int_{\Gamma_{f}^{k}} \boldsymbol{u}_{3 D}^{(k)} \cdot \boldsymbol{n} d \gamma=Q_{0 D}^{(k-1)}
\end{aligned}
$$

2. SOLVE OD problem

$$
\begin{aligned}
& \mathcal{P}_{0 D}\left(\widetilde{Q}_{0 D}^{(k)}\right)=0, \\
& P_{0 D}^{(k)}=\frac{1}{\left|\Gamma_{f}\right|} \int_{\Gamma_{f}} p_{3 D}^{(k)} d \gamma ;
\end{aligned}
$$

3. Relaxation: $Q_{0 D}^{(k)}=\omega \widetilde{Q}_{0 D}^{(k)}+(1-\omega) Q_{0 D}^{(k-1)}$;

4. Update: $k \rightarrow k+1$.

\section{END DO}

The convergence of these schemes can be proven by means of general abstract arguments for iterative methods. Let $\mathcal{F}$ be the abstract notation for the differential operator related to a suitable linearization of the fluid-structure $3 \mathrm{D}$ problem $\mathcal{P}_{3 D}=0$ operating on the variables $\mathbf{v}_{3 D}$. Correspondingly, $\mathcal{B}$ represents the action of the variables of the lumped parameter models $\mathbf{v}_{0 D}$ so that at each time step $\mathcal{P}_{3 D}=0$ can be rewritten in the generic form

$$
\mathcal{F} \mathbf{v}_{3 D}+\mathcal{B} \mathbf{v}_{0 D}=\mathbf{b}_{3 D}
$$

where $\mathbf{b}_{3 D}$ collects the effect of forcing terms, time discretization terms, boundary data (with the exclusion of the interface variables) and time discretization. Similarly, we rewrite $\mathcal{P}_{0 D}=0$ in the form

$$
\mathcal{L} \mathbf{v}_{0 D}+\mathcal{D} \mathbf{v}_{3 D}=\mathbf{b}_{0 D}
$$

where $\mathcal{L}$ is the differential system of the lumped parameter model, $\mathcal{D}$ represents the action of the 
interface variables computed by the linearized $3 \mathrm{D}$ model, and $\mathbf{b}_{0 D}$ collects the external forcing and time discretization terms.

After appropriate discretization and linearization procedures, the multiscale model can be therefore written in the matrix like formulation

$$
\left[\begin{array}{ll}
\mathcal{F} & \mathcal{B} \\
\mathcal{D} & \mathcal{L}
\end{array}\right]\left[\begin{array}{l}
\mathbf{v}_{3 D} \\
\mathbf{v}_{0 D}
\end{array}\right]=\left[\begin{array}{l}
\mathbf{b}_{3 D} \\
\mathbf{b}_{0 D}
\end{array}\right]
$$

In this respect, the iterative methods illustrated in Algorithm 2 and 3 can be regarded as block relaxed Gauss-Seidel schemes. As a matter of fact, if we define the operator $\mathcal{L}_{0 D} \equiv \mathcal{L}^{-1} \mathcal{D}$ and $\mathcal{L}_{3 D} \equiv \mathcal{F}^{-1} \mathcal{B}$, then the relaxed Gauss-Seidel method can be formulated as

$$
\mathbf{v}_{0 D}^{(k+1)}=\left[(1-\omega) \mathcal{I}+\omega \mathcal{L}_{0 D} \mathcal{L}_{3 D}\right] \mathbf{v}_{0 D}^{(k)}+\mathbf{f}_{0 D}^{(k)},
$$

for a suitable vector $\mathbf{f}_{0 D}^{(k)}$. In principle, convergence can be guaranteed by proving that for an appropriate choice of the relaxation parameter $\omega$ the operator $\left[(1-\omega) \mathcal{I}+\omega \mathcal{L}_{0 D} \mathcal{L}_{3 D}\right]$ is a contraction. In practice, it is fairly difficult to find an explicit formula for $\omega$ (generally dependent on the discretization parameters) and a trial and error approach is used. The previous analysis holds also for the 3D-1D coupling. In this case, the dependence of the optimal $\omega$ on the physical parameters of the problem has been numerically investigated in [65] .

Robin/Robin interface conditions.. In the examples above we have considered interface conditions involving pressure and velocity (converted in average pressures and flow rates for the 0D) separately. As for Domain Decomposition methods, this corresponds to selecting Dirichlet/Neumann schemes, where the velocity conditions represent Dirichlet conditions and the pressure the Neumann ones. Other conditions can be equivalently considered, for instance Robin/Robin conditions stemming from a linear combination of the Dirichlet/Neumann ones. From the standpoint of the continuous - dimensionally homogeneous - problem, all these choices are equivalent. The only requisite is that the two selected conditions are linearly independent one of the other. Conversely, from the standpoint of the approximation, the different conditions will generally lead to different numerical sequences (all sharing the same limit, though) and, most importantly, to different numerical performances. An appropriate selection of the parameters of the combination is in fact expected to accelerate the convergence of the partitioned scheme. We observe that for the hydraulic network topology, Robin conditions correspond to introducing a lumped resistance on the interface branch of the bridging region. This case has been considered in [181]. Instead, for the 3D problem, the Robin/Robin scheme would lead to a defective resistance condition (see (27) and the related paragraph).

Remark 8. Failing to fulfill the bridging region compatibility may introduce some troubles in the segregated algorithm. In fact, if the redundancy of the OD model is not properly managed, the solution of the $O D$ compartment may suffer from numerical instabilities that affect the convergence of the partitioned scheme. This is illustrated in the counterexample reported in the Appendix of [139].

Remark 9. The original basic iterative scheme can be generalized for the sake of numerical efficiency in several ways. For instance, in [21] the partitioning of the geometrical multiscale model is combined with a fractional step method for solving the fluid problem, so that the dimensional mismatch at the interface affects only one substep of the $3 D$ problem with a global mitigation of the numerical effects of the mathematical heterogeneity of the two subproblems. 
Segregated schemes as a well posedness fixed point analysis tool.. As for the theoretical aspects pointed out in the introduction to this section, bridging region compatibility is relevant to ensure the well posedness of each subdomain problem. In fact, in terms of network analysis it guarantees that the simple State Variable solution approach described in [116] can be pursued, since it implies the absence of capacitance loops and inductance cut sets. In mathematical terms, 0D models are described by Differential Algebraic Systems (DAE) of equations and bridging region compatibility guarantees that the system is of type $1[6]$, so it reduces to a standard system of ODE. On the other hand, the defective 3D problem may be completed as illustrated above (and previously in Sect. 3.1). Assuming therefore that also the 3D problem is well posed, the iterative procedure explained in the algorithms above can be used not only for achieving the numerical solution of the coupled problem, but also for proving its well posedness. As a matter of fact, the coupled solution may be regarded as the fixed point of the iterative scheme. In this perspective, existence (at least locally in time) follows from the application of fixed point theorems. In particular, in Proposition 5.1 of [181] the Schauder fixed point principle was used to analyze the case of 3D problem with rigid boundaries, after showing that (i) for suitable assumptions on the initial data and under bridging region compatibility, each subproblem is well posed; at the numerical level, this implies that the the block operators $\mathcal{L}$ and $\mathcal{F}$ are invertible; (ii) the operator $\mathcal{T}=\mathcal{L}_{0 D} \mathcal{L}_{3 D}$ is locally-in-time compact. In particular, the proposition was proved for a problem where all the data used as input to the 0D model where flow rates and the boundary conditions for the 3D one were of (defective) pressure types completed with the "do-nothing" approach. The arguments may be generalized to other cases though, see [63].

\subsection{The $1 D-O D$ case}

We discuss here about partitioned algorithms related to the time discretizaton of the 1D-0D coupled problem introduced in Section 4.3, which is formally given by (70). These algorithms are based on the iterative exchange of the interface conditions between the 1D and the 0D models. Again, the concept of bridging region should be carefully addressed since the 0D model beckons one of the two interface conditions. In particular, for the solution of (70) a "Pressure/Flow rate" scheme like the one reported in Algorithm 2 could be considered, where the pressure condition $(70 \mathrm{c})$ is prescribed to the $1 \mathrm{D}$ model and the flow rate condition (70b) to the $0 \mathrm{D}$ model. Viceversa, a "Flow rate/Pressure" scheme similar to the one reported in Algorithm 3 could be considered as well.

An explicit version of "Pressure/Flow rate" algorithm has been used in [63]. Instead, a condition on the incoming characteristic variable for the 1D model to replace the one on the pressure has been proposed in [70].

It is worth mentioning that - similarly to the 3D-0D case - a partitioned approach has also provided the framework for the well posedness analysis of this heterogeneous problem via the application of a fixed point theorem, as illustrated in [63].

\subsection{Further developments and comments}

The partitioned strategies presented in the previous subsections arise from suitable iterative methods (such as the block Gauss-Seidel method) applied to the "monolithic" coupled problems. A different approach to solve the coupled problems presented in Section 4 relies on writing an equation involving only the interface unknowns and on its iterative numerical solution (possibly after a suitable linearization). We can interpret this interface equation as the geometric heterogeneous 
counterpart of the Schur complement equation, often considered in the Domain Decomposition method [178].

In [123], a non-linear system of equations involving the interface unknowns related to the pressure and the flow rate has been derived for a general network composed by "complex" (3D or 2D) and "simple" (1D or 0D) models, with an arbitrary connectivity. Then, the authors proposed to apply to this interface equation either the Broyden method or the Newton one used in combination with GMRes - see also Sect. 6.4.2.

A similar strategy has been considered in [130], where the authors detailed the computation of the Jacobian entries (or of suitable approximations) related to the application of the Newton method to the interface equation. The same framework has been applied in [27] to an interface equation related to flow rates and total pressure.

THESE METHODS, RELYING ON THE NUMERICAL SOLUTION OF THE INTERFACE EQUATION, ARE SIMPLE TO IMPLEMENT IN THE CASE OF MULTIPLE INTERFACES, SUCH AS THOSE THAT ARISE IN COMPLEX ARTERIAL NETWORKS. As a matter of fact, they deal with arrays that corresponds to a lower dimensional space (single points) yielding therefore a substantial topological simplification.

Finally, we stress again that segregated algorithms are not the only option. Iterative methods applied directly to the monolithic coupled linearized system have been advocated in $[29,32]$.

\section{An annotated review of selected works}

In this section, we review some of the most-representative contributions to the geometric multiscale approach in the past fifteen years. For several possible coupling problems (3D-1D, 3D-0D, 1D-0D or 3D-1D-0D), we chose two papers, the former with a more theoretical focus on numerical algorithms and their performances; the latter more oriented to practical aspects and applications. In this choice we necessarily had to discard many contributions. We tried however to give an exhaustive outlook to the available literature in the previous sections.

\section{1. $3 D-1 D$ coupling}

6.1.1. "On the coupling of $3 D$ and $1 D$ Navier-Stokes equations for flow problems in compliant vessels", by L. Formaggia, J.F. Gerbeau, F. Nobile, A. Quarteroni, 2001 [65]

The coupling 3D/FSI -1D models is addressed for the first time in [65], where crucial mathematical and numerical topics are elucidated and problematic aspects - still open and debated are highlighted. In particular, the authors consider the coupling between a 3D/FSI problem with the membrane structure (5) and a 1D model with the algebraic vessel law (11). Energy estimates for the stand-alone 3D/FSI and 1D models are provided. For the 3D/FSI problem, the authors derive the energy estimate for different boundary conditions on the structure at the outlet. They consider in particular the case of absorbing boundary conditions for the structure, an effective tool to avoid spurious reflections. Referring to the notation of Section 2.1.3 and to Figure 11, this outlet condition reads

$$
\frac{\partial \widetilde{\eta}_{r}}{\partial t}-\sqrt{\frac{k G H_{s}}{\rho_{s}}} \frac{\partial \widetilde{\eta}_{r}}{\partial z}=0 . \quad \text { at } \widetilde{\Sigma}_{s, d i s t} .
$$

The estimates for the stand-alone models provide the starting point for proving the global energy estimate reported here in Proposition 1. 
For the numerical simulations, the authors resort to a partitioned algorithm (see Algorithm 1). More precisely, mean traction condition (58) is prescribed to the the 3D fluid problem, whilst 1D model is equipped with condition (74) on the incoming characteristic variable.

Results are presented both in 2D and 3D cylindrical domains with different radii and a proximal pressure impluse triggering the dynamics.

Hereafter, we report the main results highlighted by the numerical experiments.

- An explicit partitioned algorithm manages the 3D-1D coupling with stable results in the simplified settings tested.

- Condition (72b) to guarantee the continuity of the area (38) at the interface leads to numerical instabilities. Most likely this follows from the mismatch of the structure laws of the 3D/FSI and 1D models. This condition was then replaced by homogeneous Dirichlet condition in the axial direction and homogeneous Neumann conditions in the tangential one for the structure.

- Both in the 2D and in the 3D experiments, the stand-alone 3D/FSI model with standard outlet boundary conditions exhibits remarkable spurious reflections. Conversely, these reflections are highly damped in the multiscale 3D/FSI-1D model.

- In order to assess the consistency of the geometric multiscale model with the full high-fidelity one, the authors compare the results of a 2D/FSI simulation and the companion 2D/FSI-1D multiscale model where the distal half of the domain is replaced by a $1 \mathrm{D}$ system. Results pinpoint that the two models are in good agreement until the pressure wave reaches the interface between 2D and 1D subdomains (velocity and pressure mismatch in the $L^{2}$ norm being less than $2.5 \%$ and $1.5 \%$ respectively). The assessment of this consistency is clearly crucial for the reliability of the results. For this reason, this topic has been successively addressed for the case of a thick structure, in [69] for the case of a cylindrical domain, and in [72] for the case of real geometries. In the latter work, the authors stress the importance of accounting for the variations of $A_{0}$ along $z$ in the $1 \mathrm{D}$ model (that is $\frac{\partial A_{0}}{\partial z} \neq 0$ in (8b), as we have for instance for stenoses or for tapering - in this latter case $\frac{\partial A_{0}}{\partial z} \leq 0$ ) in order to guarantee the consistency.

\subsection{2. 'On the potentialities of 3d-1d coupled models in hemodynamics simulations" by P.J. Blanco,} M.R. Pivello, S.A. Urquiza, R.A. Feijóo, 2009 [32]

The authors investigate here the consistency of the 3D/FSI-1D coupled system in real geometries with a circulatory network represented by a system of 1D problems. In particular they consider the 55 arteries-network proposed in [7]. Each segment is assumed to obey the algebraic vessel law (11). A 3D/FSI problem with the membrane structure law (5) replaces different portions of the network in the different experiments An implicit partitioned scheme with 3 to 6 iterations per time step is exploited.

We report the main results highlighted by the numerical experiments.

- The first numerical experiment is intended to analyze numerically the impact of the location of the 1D segment replaced by the 3D/FSI model in the multiscale setting. For this reason, three cases are considered: (i) the $3 \mathrm{D}$ region $\mathcal{A}_{\text {full }}$ replaces a significant portion of the femoral artery; (ii) the $3 \mathrm{D}$ region $\mathcal{A}_{\text {prox }}$ replaces only the first proximal part of the same artery; (iii) the $3 \mathrm{D}$ region $\mathcal{A}_{\text {dist }}$ replaces only the distal tract of the same artery. In particular, the two 
latter domains are selected in such a way that $\mathcal{A}_{\text {dist }}$ is an almost rectilinear morphology as opposed to $\mathcal{A}_{\text {prox }}$ that is significantly curved. The three multiscale problems are eventually tested against the full 1D stand-alone model. Results are in good agreement as for the average flow and pressure. In addition, pointwise velocity in the high fidelity models is much more similar in the multiscale problems $\mathcal{A}_{\text {full }}$ and $\mathcal{A}_{\text {prox }}$ than for the fully $1 \mathrm{D}$ and the multiscale with $\mathcal{A}_{\text {dist }}$. This because the basic $1 \mathrm{D}$ model postulates a rectilinear segment - as seen in Sect. 2 - so the curvature of the femoral artery is not included in the last two cases as it is in the former two, and this significant affects the numerical results.

- In the second test, the authors compare the 1D network with a 3D/FSI-1D, where the highfidelity model is used to represent the left carotid artery. The aim is to assess te sensitivity of the 3D solution to the inflow conditions prescribed at the heart. Four typical flow waveform characterizing the flow entering the ascending aorta are employed. The results show that while these conditions have a major impact on the systemic 1D solution, the local solution in the $3 \mathrm{D}$ model (including the averaged wall shear stress) is quite insensitive to the different waveforms.

\subsection{D-OD coupling}

6.2.1. "Coupling between lumped and distributed models for blood flow problems" by A. Quarteroni, S. Ragni and A. Veneziani, 2001 [175]

As we mentioned in Sect. 1, the interplay between local and systemic dynamics in determining the boundary conditions for a numerical simulation was well evident before mathematical multiscale models were developed. In fact, in [161] a 3D rigid simulation of the cavopulmonary anastomosis was assisted by a lumped parameter model. The latter was a lumped parameter network covering the entire circulation with the role of finding boundary data to be properly prescribed to the stand-alone restricted 3D model of the region of interest. Two separate solvers were used in this way to improve the reliability of the accurate $3 \mathrm{D}$ simulation as opposed to the seminal paper [175]. The latter provides the first example of a mathematically sound coupled model, where a rigid Navier-Stokes code and a lumped parameter solver defined on non-overlapping regions work in a truly multiscale fashion. At this proof-of-concept stage the full solver is actually in $2 \mathrm{D}$, since a customized research software developed by the authors was used. The conceptual scheme for multiscale modeling is not however affected by this fact and the paper illustrates results when the systemic network has a certain level of complexity, including modeling of the heart and the valves. In particular, the strategy is to segregate the computation in the two subdomains (2D and $0 \mathrm{D})$ with an explicit time advancing. More precisely, given all the velocity and pressure quantities at a given time step,

1. the solution of the lumped parameter model is computed by using the full velocity available from the previous time step, averaged along the interface and acting as a forcing term; this obtains the average pressure at the interface;

2. the latter is used as boundary condition for the $2 \mathrm{D}$ problem. More precisely, the related 2D Navier-Stokes pressure drop problem is solved with the "do-nothing" approach; the nonlinear convective term is approximated with a semi-implicit approach.

This completes the multiscale coupling step at a given instant and the time loop moves forward (see Algorithm 5.2 for the version of this algorithm corresponding to the FSI problem). 
Notice that the topology of the lumped parameter solver is selected to be "bridging region compatible", having capacitance at the interfaces, with pressure representing a state variable for the ODE solver.

The paper presents an algebraic formulation of both the explicit and implicit formulation - based on subiterations between the two solvers at each time step. The time step is selected to guarantee stability to the semi-implicit Navier-Stokes solver. In the simplified test cases considered, the explicit solver is overall stable.

The effectiveness of the multiscale segregated solver is assessed in different tests. A first test compares the results in a $2 \mathrm{D}$ pipe region with the pressure drop prescribed by the network with the available relative Womersley solution, proving the consistency of the multiscale solution with the analytical one. Similarly, the consistency between the solution of the lumped parameter multiscale solver and a stand-alone lumped parameter systemic model is tested, showing that the multiscale model succeeds in providing accurate results in a region of interest, yet preserving an excellent quantitative assessment of the systemic circulation.

As preliminary nontrivial results, the solver is then tested on a simplified coronary by-pass anastomosis test case. The test points out the importance of a genuine multiscale modeling to capture the local and global different hemodynamics triggered by different morphological (even if simplified) features.

In spite of its simplicity, this paper provides the basis for many multiscale models relying on segregated schemes in more realistic contexts. In this respect, we mention $[119,118,136]$ applied to the Total Cavopulmonary Connection obtained as a therapy for Left Ventricle Hypoplasia Syndrome and [12] for the analysis of carotid stenosis. In the latter paper, an extensive comparison of different boundary conditions in both stand-alone and multiscale settings is carried out, pointing out the importance of the combination of local/systemic perspectives granted by multiscale coupling for the reliability of numerical simulations.

More recently, an implicit segregated solver is used in [139] for a detailed analysis of the Cavopulmonary Connection. The paper points out the negative impact that may have the violation of bridging region compatibility on the stability of the numerical solver.

\subsection{2. "On Coupling a Lumped Parameter Heart Model and a Three-Dimensional Finite Element Aorta Model" by H. Kim, I.E. Vignon-Clementel, C. Figueroa, J. Ladisa, K. Jansen, J.} Feinstein, and C. Taylor, 2009 [113]

Paper [113] presents a sophisticated implementation of the 3D-0D coupling for the reliable simulation of the aortic flow including a simplified model of the heart. The approach followed by the authors in this case is an instance of the "coupled multidomain method" introduced in [219]. As pointed out in Sect. 4.2.2, this is monolithic coupling of the 3D and the 0D model. The 0D model at the outflow is actually a 3 -element windkessel. As we have seen, a semi-analytical solution of the network is available thanks to the method of integrating factor. This is included in the variational formulation of the Navier-Stokes equation. In the spirit of the "do-nothing" approach indicated in [97], the selected variational formulation automatically includes the $0 \mathrm{D}$ available solution and enforces the interface conditions filling the gap between the 3D and 0D models.

A distinctive feature of the paper is the inclusion of the heart dynamics represented by a $0 \mathrm{D}$ model as well. A similar procedure was carried out in [68] with a 1D-0D coupling to cover the 55 largest arteries represented by $1 \mathrm{D}$ models dynamically interfaced with the left ventricle (0D). In particular, the nature of the boundary conditions for the 3D model at the interface with the heart are selected differently over the heart beat, 
1. pressure conditions with the coupled multidomain method are prescribed when the valve is open;

2. homogeneous Dirichlet conditions are prescribed when the valve is closed.

With "pressure conditions" we mean here again the inclusion in the Navier-Stokes variational formulation of data on the average pressure provided by an elastance-varying model for the ventricle. The switch of the valve between the two possible conditions is governed by

- the difference between the ventricular and the aortic pressures to change from close to open: when the ventricular pressure is greater than the aortic one, the type of boundary condition changes;

- the flow rate to change from open to close: when the flow at the interface becomes retrograde, the type of the prescribed conditions changes.

The model is applied to patient-specific aortic geometries. The detailed 3D model includes a linearly elastic deformable wall. Several simulations were carried out testing the multiscale solver under different conditions

1. rest vs exercise conditions, by reducing the downstream resistances (corresponding to vessel dilation) and shortening the heart beat duration under exercise;

2. aortic coarctation, pre vs post surgery, by reconstructing the case of an aortic coarctation and then removing it virtually.

As a complex proof of concept yet based on real cases, the paper illustrates the excellent reliability of coupled multiscale solvers to provide important quantitative tools in the investigation of cardiovascular diseases. Another important contribution of the paper is the accurate quantification of the lumped parameters of the 0D solver, based on a trial and error approach to match available measures. In fact the quantification of parameters for the network is a crucial aspect raising significant practical issues in patient-specific settings, still to be solved.

\section{3. $1 D-0 D$ coupling}

6.3.1. "Multiscale modelling of the circulatory system: a preliminary analysis", by L. Formaggia, F. Nobile, A. Quarteroni, A. Veneziani, 1999 [70]

The need of sound mathematical models and numerical methods for coupling the different spatial scales driving the hemodynamics in living organisms was firstly stressed in [210] and [180] and eventually advocated in [70] with preliminary exploring arguments. A general perspective on the different mathematical challenges represented by coupling dimensionally heterogeneous problems is envisioned (see Fig. 1 of that paper) and some specific aspects of the low-fidelity models (1D and $0 \mathrm{D})$ are addressed.

Specifically for the multiscale modeling the coupling between a 0D network and a single tract of the systemic tree representing a portion of the descending aorta described by a 1D model is explored for the first time. The 0D model accounts for the heart modeling with its four valves, the systemic and the pulmonary tree with 30 compartments. The $1 \mathrm{D}$ model is based on the algebraic vessel law (11) and Coriolis coefficient is set to be $\alpha=1$ for simplicity.

The 1D-0D coupling occurs both proximally and distally to the 1D model, that is thus "embedded" in the 0D model. A segregated scheme is adopted, with two different approaches at the two interfaces. At each time step the 1D model feeds the lumped parameter system with the flow 
rate proximally and the mean pressure distally. In turn the 0D model computes pressure and flow rate at the two respective interfaces. These quantities are properly combined so to prescribe in fact the incoming characteristic variables to the 1D model - see (74).

A summary of the results follows.

- An explicit treatment of the partitioned algorithm based on performing just one iteration per time step may be enough to obtain stable results.

- The adoption of the characteristic variables as boundary conditions for the $1 \mathrm{D}$ ending points is able to eliminate spurious boundary reflections from the 1D solution.

- A consistency check with a stand-alone 0D problem, where the 1D model is substituted by an elementary $0 \mathrm{D}$ compartment with appropriate parameters, highlights that the pressure waveforms perfectly matched at the distal interface, whereas small artifacts as reflections (not visible in the pure 0D model) occur at the proximal interface with the 1D model.

More than for the complexity of the models presented - that is indeed quite limited - this paper has the value of providing a first mathematical formulation of geometrical multiscale concepts, anticipating different problems and possible variants.

\subsection{2. "A global multiscale mathematical model for the human circulation with emphasis on the} venous system", by L.O. Muller and E.F. Toro, 2014 [143]

In this work, the authors study the entire cardiovascular system, with a particular attention to the venous system of the head and neck. The final aim is the study of neurovascular diseases linked to the venous vasculature of the head and neck.

They consider a detailed 1D model of the arterial and venous systems, with almost 100 branches for each system. A detailed description of the venous system of the head and neck is obtained by reconstructing 3D geometries with the software VMTK [4] and successively extracting the centerlines to build the related 1D network. The wall law is given by (12), with different values of $n_{1}$ and $n_{2}$ depending on the district considered. Venous valves are modeled with diodes. To date, this is one of the most complex $1 \mathrm{D}$ anatomy-based networks together with the ADAN network featuring about 2000 segments presented in [34].

For including the heart and the pulmonary circulation, a 0D model suitably coupled with the 1D model is introduced. This comprises the four heart chambers and corresponding cardiac valves, as well as a simplified description of the arteries and veins in the pulmonary circulation, arterioles, capillaries, and venules. These are in fact artery-vein connections coupling the arterial and the venous systems and are modeled by means of $0 \mathrm{D}$ compartments with different characteristics, so as to consider different type of connections (e.g. distribution of flow from a single artery to multiple veins, or more arteries feeding a single vein).

For the numerical solution of the coupled 1D-0D problem, the authors use a partitioned algorithm where the $1 \mathrm{D}$ model provides mean pressure terms to the $0 \mathrm{D}$ system, and it receives the incoming characteristics based on the lumped parameter results. An explicit procedure is proven to be enough to provide stable and accurate results, as in [70].

In what follows we report the main results obtained by the authors.

- Agreement with physiological values in the heart and artery system is excellent. Specifically: (a) pressure variation and volume curves well represent physiological conditions in atria and 
ventricles. (b) also the results obtained in the major arteries (aorta, legs arteries, internal carotid, basilar and vertebral arteres) are in good agreement with data reported in the literature; (c) the values of computed pressures in the arterioles, capillaries, and venules varied around physiological values $(40-60,20-30$, and 13-17 $\mathrm{mmHg}$, respectively); (d) results obtained in the venous system were found to be accurate when compared with PC-MRI flow data retrieved in the neck; In particular, numerical results obtained in the systemic venous system capture the biphasic behavior characterizing the hemodynamic in these vessels, where two marked peaks in both pressure and flow are present.

- The model does not include respiration, gravity and tone regulations. However, in view of the very good agreement with experimental data, the authors argue that these features have probably a minor role, at least when simulating rest supine position. Possible techniques to account for these aspects and preliminary numerical results are nevertheless discussed.

\subsection{D-1D-0D coupling}

\subsection{1. "Large scale simulation of the human arterial tree" by Grinberg et al., 2009 [88]}

Even though the complete coupling of all the three levels all together, 3D,1D,0D, was early prospected in [70], in practice it has been implemented in relatively few contributions. In spite of its solid mathematical foundation, the coupling of the three - so mathematically diverse - models raises some practical issues and the additional effort is not always justified by the applications. For instance, when the $3 \mathrm{D}$ component is assumed to be rigid, in many cases a direct interface with $0 \mathrm{D}$ models for the systemic circulation is preferred, since propagative dynamics are not the main focus. On the other hand, when the simulation of the pressure wave propagation is the main concern, 3D/FSI-1D models are preferred, possibly with simplified terminal boundary conditions yet subject to a trivial 0D interpretation, as we have illustrated in Sect. 4.2.2. Alternatively, when the systemic dynamics is of major interest, 1D-0D models may be enough.

In some cases (see Sect. 4.1), 0D models have been used in 3D-1D coupling for representing a specific feature missed in the 3D models - like, e.g., the compliance - rather than for representing a particular peripheral compartment, see [157].

Nevertheless, we mention the paper [88] for providing a detailed analysis of the computational costs of a complete coupling.

In particular, two aspects are peculiar of the approach presented by the authors.

1. The spectral element method is used for simulating the fluid dynamics in 3D. This is advocated as alternative to the finite element method and provides a trade off between the accuracy (given by spectral methods) and the geometrical versatility (yielded by finite element decomposition).

2. Multilevel partitioning of the arterial tree is necessary for an efficient numerical solution of the problem. This means that two layers of subdomains are envisioned, (i) an "external" layer of subdomains coupled by an explicit enforcement of the interface conditions , (i) an "inner" level where each domain of the external partition is split into a number of strongly coupled subdomains. Computational facilities employed to solve this include thousands of parallel processors. The efficient exploitation of such facilities demands 4-5 layers of processors groups on a single computer or 5-6 when on grids.

The combination of highly performing parallel facilities and high order methods allows the 3D simulation of large portions of the vasculature, even if typically only the fluid part. Yet, this is not 
enough even in principle for solving the entire 3D system, with an estimated number of grid points (for the fluid) of 85.5 billions, out of scale for petaflop computers. Not to mention the substantial impossibility of retrieving geometrical data below a certain spatial scale. The need of properly coupling methods for including the other regions of the circulatory system is clearly pointed out on the basis of the imaging discriminant: what can be reconstructed from patient-specific images being "super-pixel" sized is treated as a 3D model, what is sup-pixel dimensioned can be solved only with low-fidelity models. Under this perspective, the following nomenclature is introduced.

- MaN, standing for Macroscopic Network, is used to identify the vessels with a diameter larger than $0.5 \mathrm{~mm}$, where a complete Navier-Stokes model can be solved after a proper patientspecific image processing. In particular, the authors present a 3D parallel simulation of the cerebral vasculature including 65 rigid vessels with 4 inlets, 31 outlets, by using 3265 CPUs with simulations of polynomial order 5 .

- MeN, standing for Mesoscopic Network, for vessels with diameter ranging down to $10 \mu \mathrm{m}$ radius. In these regions $1 \mathrm{D}$ models are considered as appropriate, spanning small arteries and arterioles in a number of 10 millions.

- MiN, standing for Microvascular Network, for the capillary bed comprising billions of arteries. Here the compartment representation is necessary. Lumped parameter models can be used as a closure approach for terminal conditions to 1D models. When the MeN covers a large number of arteries, an homogenization of the bed based on the Darcy Law can be used as well (see also [51]).

Although not providing any specific example of coupling of the three levels, the authors give a reliable estimate of computational costs required with the aim of simulating the three levels with the suggested cut-offs ( $5 \mathrm{~mm}$ radius for $\mathrm{MaN}, 10 \mu \mathrm{m}$ for $\mathrm{MeN}$ ) as a part of the Virtual Physiological Human (VPH - http://www.physiome.org) project. For instance, if MaN includes 100 arteries of the cerebral vasculature, this will require 27.7 wall-clock hours per cardiac cycle on 40,000 processors. Within the same time 10 millions of arterioles modeled by $1 \mathrm{D}$ models branching according to the Murray's law can be solved with a discontinuous Galerkin method. Likewise, 30,000 processors are required for covering the capillary beds in the same time with a Darcy empirical model. The memory requirements for this simulation range from 100 to 500 Terabytes, depending on the accuracy of the solver. These numbers apply to the cerebral districts and need to be multiplied by a factor of 10 for the entire circulation. In summary, 110,000 processors are estimated to be required for a 1-day-per-cardiac-cycle simulation, using however a memory considered out of scale at the date of the paper.

Up to date, the significance of these numbers for 2015 is not only to stress the intrinsic extreme complexity of the problem, as stated in the final sentence "there is much more modeling complexity to be added in the VPH, including blood rheology, biochemistry, blood-endothelium interactions etc., which will make such full-scale simulations intractable even on the next generation of hexascale computers". This statement also stresses how important is the judicious modeling of the vessel network for clinical purposes. If the dimensionally heterogeneous coupling is to date the only possible way to deal with these problems, fortunately in many applications we do not need this level of complexity, since the interest for a specific pathology calls for a focal quantitative analysis. The appropriate selection of the different regions to be described by the different models is crucial anyway to have both reliable and timely simulations. 


\subsection{2. "A black-box decomposition approach for coupling heterogeneous components in hemodynam-}

ics simulations" by P.J. Blanco, J. Leiva and G. Buscaglia, 2013 [31]

After a first proof of concept in [33] where the three different components are assembled by a block Gauss-Seidel approach, P.J. Blanco and coauthors propose an effective strongly modular approach in [31]. The three levels of modeling are here denoted by HP (High-Pressure), LP (LowPressure) and SV (Single Vessel). The first one is basically described by 1D models completed by windkessel elements as terminal conditions; the second one is described by 0D networks; and the last one is represented by 3D models, including the Navier-Stokes equations and the independent rings as a simple structural membrane model (see $[112,177]$ ).

The motivation given is that the three components have not only a different space scale, but different numerical features that it is worth treating in a modular or "black-box" fashion. The advantages of this approach pointed out here are that

- the codes of the different moduli do not need to be accessed to run the solver (henceforth improving code readability and maintainability);

- legacy well tested codes for each subproblem can be exploited;

- the effects of nonlinearities numerically reflected by iterations of proper linearization methods are confined and managed within a single modulus;

- the different time scales that guarantee numerical stability to the different moduli can be handled separately, with an overall efficiency gain.

The dimensionally heterogeneous solver is obtained by coupling the different moduli prescribing continuity of the interface variables and the corresponding fluxes. The defective problems are managed by a variational "do-nothing" approach and the interfaces with 0D models obey the bridging region compatibility that we addressed in Sect. 5.3 (even if this denomination is not used in [31]). For the sake of a strong modular approach, each modulus is regarded as an inputoutput relation between the interface variables. Then, a Broyden-like method for solving nonlinear problems is used for the numerical coupling. This approach relies on the correction of the current solution of a generic equation by computing its residual. For heterogeneous models, this results in solving the different moduli individually - with a specific time step - while the synchronization required by the Broyden algorithm is performed according to a global time step (larger than the modular ones). Inner iterations of the solvers of each component take care of the nonlinearities of the single modulus, while the Broyden outer iterations manage the coupling.

The approach is tested on three cases, (i) a 1D-0D model of the entire circulation; (2) a 3D1D-0D model related to the cerebral circulation with a patient-specific model of an aneurysm; (3) a 3D-1D-0D model of the arm. The results confirm the efficiency of the modularity approach. In particular, substepping for the lumped parameter (closed loops) components allows a flexible management of nonlinearities as opposed to a monolithic approach. An appropriate tuning of the parameters of the numerical discretization/linearization can in fact reduce significantly the computational time of a monolithic solver depending on the nature of the 0D model. In addition, the number of Broyden iterations is pretty insensitive to the number of interface variables; this property guarantees highly scalability properties of the heterogeneous solver.

The combination of the modular approach with the Broyden framework for outer iterations seems therefore to be one of the most effective methods for using dimensionally heterogeneous solvers in clinical applications. 


\section{Conclusions}

When translating mathematical models into real practical applications the gap between theory and practice comes into play and our sins - so to say with the proverb - find us out. This is particularly sensible for life sciences, when the constraints determining the gap have a diverse nature - from practical to ethical. It is reasonable to think that some of those constraints will be dropped or at least reduced but some other will always stand. For the successful impact of numerical methods it is crucial not to give up with mathematical rigor when filling the gap.

Impact of numerical methods in cardiovascular sciences ultimately means providing tools for quantitative analysis to be used in the clinical routine and for the decision-making process. This scenario implies an additional constraint: the computational time needs to be fitted - or more realistically compressed - into the clinical and sometimes emergency timelines. Computer Aided Clinical Trials (CACT) are now a reality [208], as large clinical studies are progressively supported by numerical models to complement data retrieved from patients in traditional manners. Reliability and efficiency are both fundamental and competing issues. As we have pointed out in the previous sections, geometric multiscale models provided the solution for filling the gap between available/measurable data and all the information required to make mathematical models theoretically sound. Enhanced computational power, albeit expected, will not guarantee to obtain patient-specific full model (3D) analysis for years. Dimensionally heterogeneous models have provided the appropriate versatile solution to be calibrated to diverse clinical problems. This has been testified by the formidable methodological developments over the years summarized in this paper as well as by several demonstrations in the literature that are a robust proof-of-concept and sometimes go beyond. As Fig. 1 in [70] was prepared to illustrate our prospected vision in a file called "dream.fig", we can certainly state that the dream came true. In addition, these studies have provided indications for problems in other fields, yet requiring dimensional heterogeneous modeling $[137,51,30,218,134,189,215]$ - just to mention some.

However, CACT and the clinical environments are raising several challenges still demanding for the development of mathematical tools as well the proper exploitation of infrastructures. In particular, we highlight two aspects that we think may be decisive for the overall impact of computational hemodynamics and geometric multiscale models on healthcare and society.

- Patient-specific parameter estimation. As also highlighted by the present work, reduced models features parameters that surrogate different aspects of the dynamics of interest. A precise patient-specific quantification of those parameters is not trivial, since it is not obtained by direct measurements of physical quantities. The accurate quantification can be obtained by a combination of measures and numerical techniques that goes under the more general name of data assimilation [213]. There are different approaches to attack this problem, from stochastic-based methods like the Kalman filter to variational techniques possibly paired with model reduction, see e.g. $[22,20,19,225,107]$. These methods are to date quite computationally intensive and often do not fit into timelines of medical interest, basically for the intrinsic nonlinearity of the problems at hand and the large number of parameters that need to be estimated. Trial and error approaches based on empirical adjustments of parameters available from the literature are usually preferred. The definition of rigorous and effective methods to achieve multiparameter estimation is in this context a major challenge for the years to come.

- Heterogeneous platforms management. Local clusters may not be adequate to deliver the 
computational needs of the quantitative analysis of large numbers of patients. Alternative solutions like grids and on-demand cloud resources may be the answer. In fact, it is not realistic to prospect that hospitals and healthcare facilities will equip with High Performance Computing resources, they will rather outsource the needed services. The efficient workload splitting over heterogeneous architectures for CFD in hemodynamics raises nontrivial problems in terms of efficiency and cost evaluation [195, 196, 158, 92]. The interplay between heterogeneous architectures and heterogeneous geometric multiscale models partially addressed in [88], need to be investigated in more detail for the effective deployment of infrastructures to assist the clinical activity.

From the perspective of scientific research, geometric multiscale modeling of the circulation has triggered in the years truly interdisciplinary efforts with a combination of biology, medicine, radiology and imaging sciences, mathematics (both theoretical and applied) and computer science. The role of rigorous mathematical tools is central. The appropriate formulation of problems with different geometric scales is in fact fundamental for overcoming the "insuperable difficulties" of the circulation (using Euler words) that still challenge the most modern computing architectures. Also, it clearly demonstrates that the gap between theory and practice can be actually filled by converting empirical engineering ideas into rigorous numerical methods. It is worth remembering how this research strongly relies on the contribution of "giant" mathematicians like L. Euler. Without the incredibly pioneering Euler's intuitions for representing the blood circulation none of the present geometric multiscale numerical models would have been possible.

The challenge of computational hemodynamics to cardiovascular diseases is on, the progressive refinement of methodologies and technologies gives more than a reason to get hope - and numerical mathematics of multiscale models had, has and will have to play a fundamental part in this.

\section{Acknowledgments}

AV wants to acknowledge the support of the National Science Foundation Grants DMS 1419060 and DMS 1412963 projects, Georgia Research Alliance, The Coulter Foundation, Emory University Research Committee 2015, Abbott Inc. and Fondazione Cariplo (Italy) for the support given relevant for the topics presented here. $\mathrm{CV}$ and $\mathrm{AQ}$ has been partially supported by the Italian MIUR PRIN09 project no. 2009Y4RC3B 001. The research of AQ was also supported by the Swiss National Science Foundation (SNF), project no. 140184.

\section{References}

[1] M. Aletti, A. Bortolossi, S. Perotto, and A. Veneziani. One-dimensional surrogate models for advection-diffusion problems. In Numerical Mathematics and Advanced ApplicationsENUMATH 2013, pages 447-455. Springer, 2015.

[2] D. Amadori, S. Ferrari, and L. Formaggia. Derivation and analysis of a fluid-dynamical model in thin and long elastic vessels. Networks and Heterogeneous Media, 2(1):99-125, 2007.

[3] L. Antiga, J. Peiró, and D.A. Steinman. From image data to computational domains. In Cardiovascular mathematics, edited by A. Quarteroni, L. Formaggia, A. Veneziani, Chapter 4, pages 123-175. Springer, 2009. 
[4] L. Antiga and D. Steinman. The vascular modeling toolkit (VMTK), 2009.

[5] L. Antiga, B.A. Wasserman, and D.A. Steinman. On the overestimation of early wall thickening at the carotid bulb by black blood mri, with implications for coronary and vulnerable plaque imaging. Magnetic Resonance in Medicine, 60(5):1020-1028, 2008.

[6] U.M. Ascher and L.R. Petzold. Computer methods for ordinary differential equations and differential-algebraic equations, volume 61. Siam, 1998.

[7] A. P. Avolio. Multi-branched model of the human arterial system. Medical and Biological Engineering and Computing, 18:709-718, 1980.

[8] K. Azer and C.S. Peskin. A one-dimensional model of blood flow in arteries with friction and convection based on the Womersley velocity profile. Cardiovasc Eng, 7:51-73, 2007.

[9] S. Badia, F. Nobile, and C. Vergara. Fluid-structure partitioned procedures based on Robin transmission conditions. J. Comput. Physics, 227:7027-7051, 2008.

[10] S. Badia, F. Nobile, and C. Vergara. Robin-Robin preconditioned Krylov methods for fluidstructure interaction problems. Comput. Methods Appl. Mech. Engrg., 198(33-36):2768-2784, 2009 .

[11] S. Badia, A. Quaini, and A. Quarteroni. Modular vs. non-modular preconditioners for fluid-structure systems with large added-mass effect. Comput. Methods Appl. Mech. Engrg., 197:4216-4232, 2008.

[12] R. Balossino, G. Pennati, F. Migliavacca, L. Formaggia, A. Veneziani, M. Tuveri, and G. Dubini. Computational models to predict stenosis growth in carotid arteries: which is the role of boundary conditions? Comput Methods Biomech Biomed Engin., 12(1):113-123, 2009.

[13] A. Barker and X.C. Cai. Scalable parallel methods for monolithic coupling in fluid-structure interaction with application to blood flow modeling. J. Comput. Phys., 229:642-659, 2010.

[14] A.C.L. Barnard, W.A. Hunt, W.P. Timlake, and E. Varley. A theory of fluid flow in compliant tubes. Biophysical journal, 6(6):717-724, 1966.

[15] Y. Bazilevs, V.M Calo, Y. Zhang, and T.J.R Hughes. Isogeometric fluid-structure interaction analysis with applications to arterial blood flow. Computational Mechanics, 38(4-5):310-322, 2006.

[16] Y. Bazilevs, J.R. Gohean, T.J.R. Hughes, R.D. Moser, and Y. Zhang. Patient-specific isogeometric fluid-structure interaction analysis of thoracic aortic blood flow due to implantation of the jarvik 2000 left ventricular assist device. Computer Methods in Applied Mechanics and Engineering, 198(45):3534-3550, 2009.

[17] Y. Bazilevs, K. Takizawa, and T.E. Tezduyar. Computational fluid-structure interaction: methods and applications. John Wiley \& Sons, 2012.

[18] H. Beirao da Veiga. On the existence of strong solutions to a coupled fluid-structure evolution problem. Journal of Mathematical Fluid Mechanics, 6(1):21-52, 2004. 
[19] L. Bertagna, M. D'Elia, M. Perego, and A. Veneziani. Data assimilation in cardiovascular fluid-structure interaction problems: An introduction. In Fluid-Structure Interaction and Biomedical Applications, pages 395-481. Springer, 2014.

[20] L. Bertagna and A. Veneziani. A model reduction approach for the variational estimation of vascular compliance by solving an inverse fluid-structure interaction problem. Inverse Problems, 30(5), 2014.

[21] C. Bertoglio, A. Caiazzo, and M. Fernández. Fractional-step schemes for the coupling of distributed and lumped models in hemodynamics. SIAM J Sc Comp, 35(3):B551-B575, 2013.

[22] C. Bertoglio, P. Moireau, and J.F. Gerbeau. Sequential parameter estimation for fluidstructure problems: Application to hemodynamics. International Journal for Numerical Methods in Biomedical Engineering, 28(4):434-455, 2012.

[23] D. Bessems, M. Rutten, and F.N. Van De Vosse. A wave propagation model of blood flow in large vessels using an approximate velocity profile function. J. Fluid Mech., 580:145-168, 2007.

[24] P. Blanco, M. Discacciati, and A. Quarteroni. Modeling dimensionally-heterogeneous problems: analysis, approximation and applications. Numer. Math., 119:299-335, 2011.

[25] P.J. Blanco, L.A. Mansilla Alvarez, and R.A. Feijóo. Hybrid element-based approximation for the Navier-Stokes equations in pipe-like domains. Computer Methods in Applied Mechanics and Engineering, 283:971 - 993, 2015.

[26] P.J. Blanco, G.D. Ares, S.A. Urquiza, and R.A. Feijóo. On the effect of preload and prestretch on hemodynamic simulations: an integrative approach. Biomechanics and Modeling in Mechanobiology, 2015.

[27] P.J. Blanco, S. Deparis, and A.C.I. Malossi. On the continuity of mean total normal stress in geometrical multiscale cardiovascular problems. J Comp Phys, 51:136-155, 2013.

[28] P.J. Blanco and R.A. Feijóo. A dimensionally-heterogeneous closed-loop model for the cardiovascular system and its applications. Medical Engineering 83 Physics, 35(5):652667, 2013.

[29] P.J. Blanco, R.A. Feijóo, and S.A. Urquiza. A unified variational approach for coupling 3d-1d models and its blood flow applications. Comput. Methods Appl. Mech. Engrg., 196:4391-4410, 2007.

[30] P.J. Blanco, R.A. Feijóo, and S.A. Urquiza. A variational approach for coupling kinematically incompatible structural models. Comput. Methods Appl. Mech. Engrg., 197(17-18):1577$1602,2008$.

[31] P.J. Blanco, J.S. Leiva, and G.C. Buscaglia. A black-box decomposition approach for coupling heterogeneous components in hemodynamics simulations. International journal for numerical methods in biomedical engineering, 29(3):408-427, 2013.

[32] P.J. Blanco, M.R. Pivello, S.A. Urquiza, and R.A. Feijóo. On the potentialities of 3d-1d coupled models in hemodynamics simulations. J Biomech, 42:919-930, 2009. 
[33] P.J. Blanco, S.A. Urquiza, and R.A. Feijóo. Assessing the influence of heart rate in local hemodynamics through coupled 3d-1d-0d models. Int J Num Meth Biomed Eng, 26:890-903, 2010 .

[34] P.J. Blanco, S.M. Watanabe, M.A.R.F. Passos, Lemos P., Feijóo R.A., et al. An anatomically detailed arterial network model for one-dimensional computational hemodynamics. Biomedical Engineering, IEEE Transactions on, 62(2):736-753, 2015.

[35] T. Bodnár, G.P. Galdi, and Š. Nečasová. Fluid-Structure Interaction and Biomedical Applications. Springer, 2014.

[36] J.M. Boese, M. Bock, S.O. Schoenberg, and L.R. Schad. Estimation of aortic compliance using magnetic resonance pulse wave velocity measurement. Physics in Medicine and Biology, 45(6):1703-1713, June 2000. PMID: 10870719.

[37] D. Boffi and L. Gastaldi. A finite element approach for the immersed boundary method. Comp. and Struct, 81(8-11):491-501, 2003.

[38] E. Boileau, P. Nithiarasu, P.J. Blanco, L.O. Muller, F.E. Fossan, L.R. Hellevik, W.P. Donders, W. Huberts, M. Willemet, and J. Alastruey. A benchmark study of numerical schemes for one-dimensional arterial blood flow modelling. International journal for numerical methods in biomedical engineering, DOI: 10.1002/cnm.2732, 2015.

[39] J. Bols, J. Degroote, B. Trachet, B. Verhegghe, P. Segers, and J. Vierendeels. A computational method to assess the in vivo stresses and unloaded configuration of patient-specific blood vessels. Journal of Computational and Applied Mathematics, 246:10-17, 2013.

[40] W. Boyce and R.C. Di Prima. Elementary differential equations and boundary value problems. John Wiley and Sons. Inc. New York, hal, 511, 2008.

[41] C.H. Bruneau and P. Fabrie. Effective downstream boundary conditions for incompressible NavierStokes equations. Int J Num Meth Fl, 19(8):963-705, 1994.

[42] C.H. Bruneau and P. Fabrie. New efficient boundary conditions for incompressible NavierStokes equations: a well-posedness result. Rairo, 30(7):815-840, 1996.

[43] I.C. Campbell, J. Ries, S.S. Dhawan, A.A. Quyyumi, W.R. Taylor, and J.N. Oshinski. Effect of inlet velocity profiles on patient-specific computational fluid dynamics simulations of the carotid bifurcation. J Biomech Eng, 134(5):051001, 2012.

[44] T.E. Carew, R.N. Vaishnav, and D.J. Patel. Compressibility of the arterial wall. Circ Res, 23:61-68, 1968.

[45] P. Causin, J.F. Gerbeau, and F. Nobile. Added-mass effect in the design of partitioned algorithms for fluid-structure problems. Comput. Methods Appl. Mech. Engrg., 194(42-44):4506$4527,2005$.

[46] C.M. Colciago, S. Deparis, and A. Quarteroni. Comparisons between reduced order models and full 3d models for fluidstructure interaction problems in haemodynamics. Journal of Computational and Applied Mathematics, 2754:120-138, 2014. 
[47] C. Conca, C. Pares, O. Pironneau, and M. Thiriet. Navier-Stokes equations with imposed pressure and velocity fluxes. International Journal for Numerical Methods in Fluids, (20(4)):267-287, 1995.

[48] W. Cousins and P.A. Gremaud. Boundary conditions for hemodynamics: The structured tree revisited. J Comp Phys, 231(18):6086-6096, 2012.

[49] P. Crosetto. Fluid-Structure Interaction Problems in Hemodynamics: Formulation, Solver, Preconditioners and Applications. PhD thesis, Ecole Polytechnique Fédérale de Lausanne, 2011.

[50] P. Crosetto, S. Deparis, G. Fourestey, and A. Quarteroni. Parallel algorithms for fluidstructure interaction problems in haemodynamics. SIAM J. Sci. Comput., 33:1598-1622, 2011.

[51] C. D'Angelo and A. Quarteroni. On the coupling of $1 \mathrm{~d}$ and 3d diffusion-reaction equations: Application to tissue perfusion problems. Mathematical Models and Methods in Applied Sciences, 18(08):1481-1504, 2008.

[52] S. de Putter, B.J.B.M. Wolters, M.C.M. Rutten, M. Breeuwer, F.A. Gerritsen, and F.N. van de Vosse. Patient-specific initial wall stress in abdominal aortic aneurysms with a backward incremental method. Journal of Biomechanics, 40:1081-1090, 2007.

[53] J. Degroote, K.J. Bathe, and J. Vierendeels. Performance of a new partitioned procedure versus a monolithic procedure in fluid-structure interaction. Computer \& Structures, 1112(87):793-801, 2009.

[54] M. D'Elia, L. Mirabella, T. Passerini, M. Perego, M. Piccinelli, C. Vergara, and A. Veneziani. Applications of variational data assimilation in computational hemodynamics, volume Modeling of Physiological Flows. Springer, 2011.

[55] M. D'Elia, M. Perego, and A. Veneziani. A variational data assimilation procedure for the incompressible Navier-Stokes equations in hemodynamics. J. Sc. Comp., 52(2):340-359, 2012 .

[56] M. D'Elia and A. Veneziani. Uncertainty quantification for data assimilation in a steady incompressible Navier-Stokes problem. ESAIM: Mathematical Modelling and Numerical Analysis, 47(04):1037-1057, 2013.

[57] S. Deparis, D. Forti, G. Grandperrin, and A. Quarteroni. Facsi: A block parallel preconditioner for Fluid-Structure interaction in hemodynamics. MATHICSE Technical Report, 13, 2015.

[58] J. Donea. An arbitrary Lagrangian-Eulerian finite element method for transient dynamic fluid-structure interaction. Comput. Methods Appl. Mech. Engrg., 33:689-723, 1982.

[59] V.J. Ervin and H. Lee. Numerical approximation of a quasi-newtonian Stokes flow problem with defective boundary conditions. SIAM J. Numer. Anal., 45(5):21202140, 2007. 
[60] L. Euler. Principia pro motu sanguinis per arterias determinando. Opera posthuma mathematica et physica anno 1844 detecta. Ediderunt P.H. Fuss et N. Fuss Petropoli; Apund Eggers et Socios., 1:814-823, 1775.

[61] M.A. Fernandez and J.F. Gerbeau. Algorithms for fluid-structure interaction problems. In Cardiovascular mathematics, edited by A. Quarteroni, L. Formaggia, A. Veneziani, Chapter 9, pages 307-346. Springer, 2009.

[62] M.A. Fernández, J.F. Gerbeau, and C. Grandmont. A projection semi-implicit scheme for the coupling of an elastic structure with an incompressible fluid. Int. J. Num. Methods Engrg., 69(4):794-821, 2007.

[63] M.A. Fernàndez, V. Milisic, and A. Quarteroni. Analysis of a geometrical multiscale blood flow model based on the coupling of odes and hyperbolic pdes. Multiscale Model Simul, $4(1): 215-236,2005$.

[64] C.A. Figueroa, I.E. Vignon-Clementel, K.E. Jansen, T.J.R Hughes, and C.A. Taylor. A coupled momentum method for modeling blood flow in three-dimensional deformable arteries. Computer Methods in Applied Mechanics and Engineering, 195(41-43):5685-5706, 2006.

[65] L. Formaggia, J.F. Gerbeau, F. Nobile, and A. Quarteroni. On the coupling of 3D an 1D Navier-Stokes equations for flow problems in compliant vessels. Comput. Methods Appl. Mech. Engrg., 191(6-7):561-582, 2001.

[66] L. Formaggia, J.F. Gerbeau, F. Nobile, and A. Quarteroni. Numerical treatment of defective boundary conditions for the Navier-Stokes equation. SIAM J. Numer. Anal., 40(1):376-401, 2002 .

[67] L. Formaggia, D. Lamponi, and A. Quarteroni. One-dimensional models for blood flow in arteries. Journal of Engineering Mathematics, 47(3-4):251-276, 2003.

[68] L. Formaggia, D. Lamponi, M. Tuveri, and A. Veneziani. Numerical modeling of 1d arterial networks coupled with a lumped parameters description of the heart. Computer Methods in Biomechanics and Biomedical Engineering, 9(5):273-288, 2006.

[69] L. Formaggia, A. Moura, and F. Nobile. On the stability of the coupling of 3d and 1d fluidstructure interaction models for blood flow simulations. M2AN Math. Model. Numer. Anal., 41(4):743-769, 2007.

[70] L. Formaggia, F. Nobile, A. Quarteroni, and A. Veneziani. Multiscale modelling of the circulatory system: a preliminary analysis. Comput and Visual in Science, 2:75-83, 1999.

[71] L. Formaggia, A. Quarteroni, and A. Veneziani (Eds.). Cardiovascular Mathematics - Modeling and simulation of the circulatory system. Springer, 2009.

[72] L. Formaggia, A. Quarteroni, and C. Vergara. On the physical consistency between threedimensional and one-dimensional models in haemodynamics. J. Comput. Physics, 244:97$112,2013$. 
[73] L. Formaggia, A. Veneziani, and C. Vergara. A new approach to numerical solution of defective boundary value problems in incompressible fluid dynamics. SIAM J. Numer. Anal., 46(6):2769-2794, 2008.

[74] L. Formaggia, A. Veneziani, and C. Vergara. Flow rate boundary problems for an incompressible fluid in deformable domains: formulations and solution methods. Comput. Methods Appl. Mech. Engrg., 199 (9-12):677-688, 2009.

[75] L. Formaggia and C. Vergara. Prescription of general defective boundary conditions in fluiddynamics. Milan Journal of Mathematics, 80(2):333-350, 2012.

[76] C. Forster, W. Wall, and E. Ramm. Artificial added mass instabilities in sequential staggered coupling of nonlinear structures and incompressible viscous flow. Comput. Methods Appl. Mech. Engrg., 196(7):1278-1293, 2007.

[77] O. Franck. The basic shape of the arterial pulse. first treatise: mathematical analysis. 1899. J. Mol. Cell. Cardiol., 22(3):255-277, 1990.

[78] Y. Fung. Biomechanics: mechanical properties of living tissues. Springer, 1993.

[79] Y. C. Fung, K. Fronek, and P. Patitucci. Pseudoelasticity of arteries and the choice of its mathematical expression. American Journal of Physiology, 237(5):H620-H631, 1979.

[80] K. Galvin and H. Lee. Analysis and approximation of the cross model for quasi-newtonian flows with defective boundary conditions. Appl. Math. Comp., 222:244-254, 2013.

[81] K. Galvin, H. Lee, and L.G. Rebholz. Approximation of viscoelastic flows with defective boundary conditions. J. Non Newt. Fl. Mech., 169-170:104-113, 2012.

[82] M.W. Gee, U. Kuttler, and W.A. Wall. Truly monolithic algebraic multigrid for fluidstructure interaction. Int. J. Num. Methods Engrg., 85(8):987-1016, 2011.

[83] M.W. Gee, C. Reeps, H.H. Eckstein, and W.A. Wall. Prestressing in finite deformation abdominal aortic aneurysm simulation. Journal of Biomechanics, 42:1732-1739, 2009.

[84] G. Gigante and C. Vergara. Analysis and optimization of the generalized schwarz method for elliptic problems with application to fluid-structure interaction. Numer Math, 131(2):369404, 2015.

[85] L. Gerardo Giorda, F. Nobile, and C. Vergara. Analysis and optimization of robin-robin partitioned procedures in fluid-structure interaction problems. SIAM J. Numer. Anal., 48(6):2091-2116, 2010.

[86] C. Grandmont. Analyse Mathematique et Numerique de Quelques Problemes d Interaction Fluide-Structure. PhD thesis, Laboratoire d Analyse Numrique de Paris VI, 1998.

[87] A.E. Green, P.M. Naghdi, and M. J. Stallard. A direct theory of viscous fluid flow in pipes II. Flow of incompressible viscous fluid in curved pipes. Philosophical Transactions of the Royal Society of London. Series A: Physical and Engineering Sciences, 342:543-572, 1993. 
[88] L. Grinberg, T. Anor, J.R. Madsen, A. Yakhot, and G.E. Karniadakis. Large-scale simulation of the human arterial tree. Clinical and Experimental Pharmacology and Physiology, 36(2):194-205, 2009.

[89] L. Grinberg, E. Cheever, T. Anor, J.R. Madsen, and G.E. Karniadakis. Modeling blood flow circulation in intracranial arterial networks: a comparative 3d/1d simulation study. Annals of Biomed Eng, 39(1):297-309, 2010.

[90] L. Grinberg and G.E. Karniadakis. Outflow boundary conditions for arterial networks with multiple outlets. Annals of Biomed Eng, 36(9):1496-1514, 2008.

[91] B. Guerciotti, C. Vergara, L. Azzimonti, L. Forzenigo, A. Buora, P. Biondetti, and M. Domanin. Computational study of the fluid-dynamics in carotids before and after endarterectomy. Journal of Biomechanics, 2015.

[92] S. Guzzetti, T. Passerini, J. Slawinsky, U. Villa, A. Veneziani, and V. Sunderam. Platform and algorithm effects on computational fluid dynamics applications in life sciences. Tr-2015006, Dept. Math. and Comp. Science, Emory Univ, Atlanta, GA, USA, 2015.

[93] C.M. Haggerty, L. Mirabella, M. Restrepo, D. A de Zélicourt, J. Rossignac, F. Sotiropoulos, T.L. Spray, K.R. Kanter, M.A. Fogel, and A.P. Yoganathan. Patient-specific surgery planning for the fontan procedure. In Computer Models in Biomechanics, pages 217-228. Springer, 2013.

[94] X. He, D.N. Ku, and J.E. Moore Jr. Simple calculation of the velocity profiles for pulsatile flow in a blood vessel using mathematica. Ann Biomed Eng, 21:45-49, 1993.

[95] G.W. Hedstrom. Nonreflecting boundary conditions for nonlinear hyperbolic systems. Journal of Computational Physics, 30(2):222-237, 1979.

[96] M. Heil. An efficient solver for the fully coupled solution of large-displacement fluid-structure interaction problems. Comput. Methods Appl. Mech. Engrg., 193:1-23, 2004.

[97] J.G. Heywood, R. Rannacher, and S. Turek. Artificial boundaries and flux and pressure conditions for the incompressible NAvier-STokes equations. Int. J. Num. Methods Fluids, 22:325-352, 1996.

[98] B. Hillen, H.W. Hoogstraten, and L. Post. A wave propagation model of blood flow in large vessels using an approximate velocity profile function. J Biomech, 19:187-194, 1986.

[99] R. Holenstein, P. Niederer, and M. Anliker. A viscoelastic model for use in predicting arterial pulse waves. J Biomech Eng, 102:318-325, 1980.

[100] G.A. Holzapfel, T.C. Gasser, and R.W. Ogden. A new constitutive framework for arterial wall mechanics and a comparative study of material models. J Elast, 61:1-48, 2000.

[101] G.A. Holzapfel and R.W. Ogden. Constitutive modelling of arteries. Proc. R. Soc. Lond. Ser. A Math. Phys. Eng. Sci., 466(2118):1551-1596, 2010.

[102] F.C. Hoppensteadt and C.S. Peskin. Mathematics in medicine and the life sciences. Springer, 1992. 
[103] M.-C. Hsu and Y. Bazilevs. Blood vessel tissue prestress modeling for vascular fluidstructure interaction simulation. Finite Elements in Analysis and Design, 47:593-599, 2011.

[104] T. J. R. Hughes, W. K. Liu, and T. K. Zimmermann. Lagrangian-Eulerian finite element formulation for incompressible viscous flows. Comput. Methods Appl. Mech. Engrg., 29(3):329$349,1981$.

[105] T.J.R. Hughes. A Study of the One-Dimensional Theory of Arterial Pulse Propagation. PhD thesis, University of California, Berkeley, 1974.

[106] T.J.R. Hughes and J. Lubliner. On the one-dimensional theory of blood flow in the larger vessels. Mathematical Biosciences, 18(1-2):161-170, 1973.

[107] L. Itu, P. Sharma, T. Passerini, A. Kamen, C. Suciu, and D. Comaniciu. A parameter estimation framework for patient-specific hemodynamic computations. Journal of Computational Physics, 281:316-333, 2015.

[108] K.P. Ivanov and E.G. Bournaski. Combined distributed and lumped parameters model for transient flow analysis in complex pipe networks. Computer methods in applied mechanics and engineering, 130(1):47-56, 1996.

[109] J. Janela, A. Moura, and A. Sequeira. Absorbing boundary conditions for a 3d non-newtonian fluid-structure interaction model for blood flow in arteries. International Journal of Engineering Science, 48(11):1332-1349, 2010.

[110] AK Jayanthy, N Sujatha, and M Ramasubba Reddy. Measuring blood flow: techniques and applications-a review. Int. J. Res. Review Appl. Sci, 6:203-216, 2011.

[111] D.A. Johnson, U.P. Naik, and A.N. Beris. Efficient implementation of the proper outlet flow conditions in blood flow simulations through asymmetric arterial bifurcations. Int. J. Num. Methods Fluids, 66(11):1383-1408, 2011.

[112] G. Karner, K. Perktold, M. Hofer, and D. Liepsch. Flow characteristics in an anatomically realistic compliant carotid artery bifurcation model. Methods in Biomechanics and Biomedical Engineering, 2(39-41):171-185, 1999.

[113] H. Kim, I.E. Vignon-Clementel, C. Figueroa, J. Ladisa, K. Jansen, J. Feinstein, and C. Taylor. On coupling a lumped parameter heart model and a three-dimensional finite element aorta model. Ann Biomed Eng, 37:2153-2169, 2009.

[114] T. Korakianitis and Y. Shi. Numerical simulation of cardiovascular dynamics with healthy and diseased heart valves. J Biomech, 39(11):19641982, 2006.

[115] R.H. Kufahl and M.E. Clark. A circle of Willis simulation using distensible vessels and pulsatile flow. J Biomech Eng, 107(2):112-122, 1985.

[116] E.S. Kuh and R.A. Rohrer. The state-variable approach to network analysis. Proceedings of the IEEE, 53(7):672-686, 1965. 
[117] U. Kuttler, M. Gee, C. Forster, A. Comerford, and W.A. Wall. Coupling strategies for biomedical fluid-structure interaction problems. Int. J. Num. Methods Biomed. Engrg., $26: 305-321,2010$.

[118] K. Laganá, R. Balossino, F. Migliavacca, G. Pennati, E.L. Bove, M.R. de Leval, and G. Dubini. Multiscale modeling of the cardiovascular system: application to the study of pulmonary and coronary perfusions in the univentricular circulation. Journal of biomechanics, 38(5):1129-1141, 2005.

[119] K. Laganá, G. Dubini, F. Migliavacca, R. Pietrabissa, G. Pennati, A. Veneziani, and A. Quarteroni. Multiscale modelling as a tool to prescribe realistic boundary conditions for the study of surgical procedures. Biorheol, 39(3-4):359-364, 2002.

[120] J.D. Lambert. Computational methods in ordinary differential equations. Chichester, New York, 2014.

[121] R. M. Lancellotti. Numerical Computations of Deflated Vascular Geometries fo FluidStructure Interaction in Haemodynamics. PhD thesis, Universit degli Studi di Napoli Federico II, July 2012.

[122] H. Lee. Optimal control for quasi-newtonian flows with defective boundary conditions. Comput. Methods Appl. Mech. Engrg., 200:2498-2506, 2011.

[123] J.S. Leiva, P.J. Blanco, and G.C. Buscaglia. Partitioned analysis for dimensionallyheterogeneous hydraulic networks. Mult Model Simul, 9:872-903, 2011.

[124] R.J. LeVeque. Numerical methods for conservation laws, volume 132. Birkhäuser Basel, 1992.

[125] F. Liang, S. Takagi, R. Himeno, and H. Liu. Multi-scale modeling of the human cardiovascular system with applications to aortic valvular and arterial stenoses. Medical $\&$ Biological Engineering \& Computing, 47(7):743-755, 2009.

[126] Y. Liu, C. Charles, M. Gracia, H. Gregersen, and G. S. Kassab. Surrounding tissues affect the passive mechanics of the vessel wall: theory and experiment. Am J Physiol Heart Circ Physiol, 293:H3290-H3300, 2007.

[127] J. Lu, X. Zhou, and M. Raghavan. Inverse elastostatic stress analysis in pre-deformed biological structures: demonstration using abdominal aortic aneurysms. Journal of Biomechanics, 40:693-696, 2007.

[128] Y. Maday. Analysis of coupled models for fluid-structure interaction of internal flows. In Cardiovascular mathematics, edited by A. Quarteroni, L. Formaggia, A. Veneziani, Chapter 8, pages 279-306. Springer, 2009.

[129] A. Mahmoud, A. El-Barkouky, H.E. Farag, J. Graham, and A. Farag. A non-invasive method for measuring blood flow rate in superficial veins from a single thermal image. In Computer Vision and Pattern Recognition Workshops (CVPRW), 2013 IEEE Conference on, pages 354-359. IEEE, 2013. 
[130] A.C.I. Malossi, P.J. Blanco, P. Crosetto, S. Deparis, and A. Quarteroni. Implicit coupling of one-dimensional and three-dimensional blood flow models with compliant vessels. Multiscale Model Simul, 11(2):474-506, 2013.

[131] A.C.I. Malossi, P.J. Blanco, and S. Deparis. A two-level time step technique for the partitioned solution of one-dimensional arterial networks. Comput. Methods Appl. Mech. Engrg.,, 237-240:212-226, 2012.

[132] V. Martin, F. Clément, A. Decoene, and J.F. Gerbeau. Parameter identification for a onedimensional blood flow model. Proceedings Cemracs, 14:174-200, 2005.

[133] K.S. Matthys, J. Alastruey, J. Peiró, A.W. Khir, P. Segers, P.R. Verdonck, K.H. Parker, and S.J. Sherwin. Pulse wave propagation in a model human arterial network: Assessment of 1-d numerical simulations against in vitro measurements. J Biomech, 40(15):3476-3486, 2007.

[134] L. Mauri, S. Perotto, and A. Veneziani. Adaptive geometrical multiscale modeling for hydrodynamic problems. In Numerical Mathematics and Advanced Applications 2011, pages 723-730. Springer, 2013.

[135] A. Melani. Adjoint-based parameter estimation in human vascular one dimensional models. $\mathrm{PhD}$ thesis, Mathematical Models and Methods in Engineering, Department of Mathematics, Politecnico di Milano, 2013.

[136] F. Migliavacca, R. Balossino, G. Pennati, G. Dubini, T.Y. Hsia, M.R. de Leval, and E.L. Bove. Multiscale modelling in biofluidynamics: application to reconstructive paediatric cardiac surgery. J Biomech, 39:1010-1020, 2006.

[137] E. Miglio, S. Perotto, and F. Saleri. Model coupling techniques for free-surface flow problems: Part i. Nonlinear Analysis: Theory, Methods \& Applications, 63(5):e1885-e1896, 2005.

[138] M.E. Moghadam, Y. Bazilevs, T.Y. Hsia, I. Vignon-Clementel, and A. Marsden. A comparison of outlet boundary treatments for prevention of backflow divergence with relevance to blood flow simulations. Computational Mechanics, 48(3):277-291, 2011.

[139] M.E. Moghadam, I.E. Vignon-Clementel, R. Figliola, and Alison L. Marsden. A modular numerical method for implicit $0 \mathrm{~d} / 3 \mathrm{~d}$ coupling in cardiovascular finite element simulations. $J$ Comp Phys, 244(1):63-79, 2013.

[140] P. Moireau, N. Xiao, M. Astorino, C. A. Figueroa, D. Chapelle, C. A. Taylor, and J.F. Gerbeau. External tissue support and fluid-structure simulation in blood flows. Biomechanics and Modeling in Mechanobiology, 11(1-2):1-18, 2012.

[141] U. Morbiducci, R. Ponzini, G. Rizzo, M. Cadioli, A. Esposito, F. De Cobelli, A. Del Maschio, F.M. Montevecchi, and A. Redaelli. In vivo quantification of helical blood flow in human aorta by time-resolved three-dimensional cine phase contrast magnetic resonance imaging. Ann Biomed Eng, 37(3):516-531, 2009.

[142] L. Muller and E.F. Toro. Well-balanced high-order solver for blood flow in networks of vessels with variable properties. Int J Num Meth Biomed Eng, 29(12):1388-1411, 2013. 
[143] L. Muller and E.F. Toro. A global multiscale mathematical model for the human circulation with emphasis on the venous system. Int J Num Meth Biomed Eng, 30(7):681-725, 2014.

[144] J. Munch, A. Veneziani, and U. Villa. A factorization method for the exact prescription of flow rate conditions in incompressible fluid dynamics. in preparation, 2015.

[145] W.W. Nichols and M.F. O'Rourke, editors. McDonald's Blood Flow in Arteries. Hodder Arnold, 2005.

[146] J.A. Nitsche. Uber ein variationsprinzip zur lozung von dirichlet-problemen bei verwendung von teilraumen, die keinen randbedingungen unterworfen sind. Abhandlungen aus dem Mathematischen Seminar der Universitat Hamburg, 36:9-15, 1970/71.

[147] F. Nobile, M. Pozzoli, and C. Vergara. Time accurate partitioned algorithms for the solution of fluid-structure interaction problems in haemodynamics. Computer $\mathscr{G}$ Fluids, 86:470-482, 2013.

[148] F. Nobile, M. Pozzoli, and C. Vergara. Inexact accurate partitioned algorithms for fluidstructure interaction problems with finite elasticity in haemodynamics. Journal of Computational Physics, 273:598-617, 2014.

[149] F. Nobile and A. Veneziani. Fluid structure interaction in blood flow problems. ZAMMJournal of Applied Mathematics and Mechanics/Zeitschrift für Angewandte Mathematik und Mechanik, 79(S1):255-258, 1999.

[150] F. Nobile and C. Vergara. An effective fluid-structure interaction formulation for vascular dynamics by generalized Robin conditions. SIAM J Sc Comp, 30(2):731-763, 2008.

[151] F. Nobile and C. Vergara. Partitioned algorithms for fluid-structure interaction problems in haemodynamics. Milan Journal of Mathematics, 80(2):443-467, 2012.

[152] M.S. Olufsen. Structured tree outflow condition for blood flow in larger systemic arteries. American journal of physiology-Heart and circulatory physiology, 276(1):H257-H268, 1999.

[153] M.S. Olufsen, C.S. Peskin, W.Y. Kim, E.M. Pedersen, A. Nadim, and J. Larsen. Numerical simulation and experimental validation of blood flow in arteries with structured-tree outflow conditions. Ann Biomed Eng, 28(11):1281-1299, 2000.

[154] J.T. Ottesen, M.S. Olufsen, and J.K. Larsen. Applied mathematical models in human physiology. SIAM, 2004.

[155] S. Pant, B. Fabréges, J.F. Gerbeau, and I.E. Vignon-Clementel. A methodological paradigm for patient-specific multi-scale cfd simulations: from clinical measurements to parameter estimates for individual analysis. International Journal for Numerical Methods in Biomedical Engineering, 30(12):1614-1648, 2014.

[156] G. Papadakis. Coupling 3d and 1d fluid-structure-interaction models for wave propagation in flexible vessels using a finite volume pressure-correction scheme. Comm Numer Meth Eng, $25(5): 533-551,2009$. 
[157] T. Passerini, M. de Luca, L. Formaggia, A. Quarteroni, and A. Veneziani. A 3d/1d geometrical multiscale model of cerebral vasculature. Journal of Engineering Mathematics, 64(4):319-330, 2009.

[158] T. Passerini, J. Slawinski, U. Villa, and V. Sunderam. Experiences with cost and utility trade-offs on iaas clouds, grids, and on-premise resources. In Cloud Engineering (IC2E), 2014 IEEE International Conference on, pages 391-396. IEEE, 2014.

[159] T.J. Pedley. The fluid mechanics of large blood vessels. Cambridge monographs on mechanics and applied mathematics: Cambridge University Press, 1980.

[160] J. Peiró and A. Veneziani. Reduced models of the cardiovascular system. In Cardiovascular mathematics, edited by A. Quarteroni, L. Formaggia, A. Veneziani, Chapter 10, pages 347394. Springer, 2009.

[161] G. Pennati, F. Migliavacca, G. Dubini, R. Pietrabissa, and M.R. de Leval. A mathematical model of circulation in the presence of the bidirectional cavopulmonary anastomosis in children with a univentricular heart. Med. Eng. Phys., 19(3):223-234, 1997.

[162] M. Perego, A. Veneziani, and C. Vergara. A variational approach for estimating the compliance of the cardiovascular tissue: An inverse fluid-structure interaction problem. SIAM J Sc Comp, 33(3):1181-1211, 2011.

[163] K. Perktold, E. Thurner, and T. Kenner. Flow and stress characteristics in rigid walled and compliant carotid artery bifurcation models. Medical and Biological Engineering and Computing, 32(1):19-26, 1994.

[164] S. Perotto. A survey of hierarchical model (Hi-Mod) reduction methods for elliptic problems. Numerical Simulations of Coupled Problems in Engineering. Series: Computational Methods in Applied Sciences, Springer, S.R. Idelsohn Ed., 33:217-241, 2014.

[165] S. Perotto, A. Ern, and A. Veneziani. Hierarchical local model reduction for elliptic problems: A domain decomposition approach. Multiscale Model Simul, 8(4):1102-1127, 2010.

[166] S. Perotto and A. Veneziani. Coupled model and grid adaptivity in hierarchical reduction of elliptic problems. J. Sci. Comput., 60:505-536, 2014.

[167] C. Peskin. Flow patterns around heart valves: A numerical method. J. Comput. Physics, 10(2):252271, 1972.

[168] M. Piccinelli, C. Vergara, L. Antiga, L. Forzenigo, P. Biondetti, and M. Domanin. Impact of hemodynamics on lumen boundary displacements in abdominal aortic aneurysms by means of dynamic computed tomography and computational fluid dynamics. Biomech Model Mechanobiol, 12(6):1263-1276, 2013.

[169] Y. Pinchover and J. Rubinstein. An introduction to partial differential equations. Cambridge University Press, 2005.

[170] R. Ponzini, C. Vergara, A. Redaelli, and A. Veneziani. Reliable cfd-based estimation of flow rate in haemodynamics measures. Ultrasound in Med. and Biol., 32(10):1545-1555, 2006. 
[171] R. Ponzini, C. Vergara, G. Rizzo, A. Veneziani, A. Roghi, A. Vanzulli, O. Parodi, and A. Redaelli. Womersley number-based estimates of blood flow rate in doppler analysis: In vivo validation by means of phase contrast magnetic resonance imaging. IEEE Transaction on Biomedical Engineering, 57(7):1807-1815, 2010.

[172] A. Porpora, P. Zunino, C. Vergara, and M. Piccinelli. Numerical treatment of boundary conditions to replace lateral branches in haemodynamics. Int. J. Numer. Meth. Biomed. Eng., 28(12):1165-1183, 2012.

[173] M. Pozzoli. Efficient partitioned algorithms for the solution of fluid-structure interaction problems in haemodynamics. $\mathrm{PhD}$ thesis, Mathematical Models and Methods in Engineering, Dipartimento di Matematica, Politecnico di Milano, 2012.

[174] A. Quarteroni and L. Formaggia. Mathematical Modelling and Numerical Simulation of the Cardiovascular System, Computational models for the human body, Handbook of Numerical Analysis, volume 12. Elsevier Science, 2004.

[175] A. Quarteroni, S. Ragni, and A. Veneziani. Coupling between lumped and distributed models for blood flow problems. Comp Vis Sc, 4(2):111-124, 2001.

[176] A. Quarteroni, R. Sacco, and F. Saleri. Numerical mathematics. Springer Berlin, 2000.

[177] A. Quarteroni, M. Tuveri, and A. Veneziani. Computational vascular fluid dynamics: Problems, models and methods. Computing and Visualisation in Science, 2:163-197, 2000.

[178] A. Quarteroni and A. Valli. Domain Decomposition Methods for Partial Differential Equations. Oxford Science Publications, 1999.

[179] A. Quarteroni and A. Veneziani. Modeling and simulation of blood flow problems. In Computational Science for the 21st Century, pages 369-379. J. Periaux et al. eds, J. Wiley and Sons, 1997.

[180] A. Quarteroni and A. Veneziani. Modeling and simulation of blood flow problems. Technical report, Bristeau, M.-O.(ed.) et al. Chichester: John Wiley \& Sons., 1997.

[181] A. Quarteroni and A. Veneziani. Analysis of a geometrical multiscale model based on the coupling of ode and pde for blood flow simulations. Multiscale Model. Simul., 1(2):173-195, 2003.

[182] M. Raghavan and D. Vorp. Towards a biomechanical tool to evaluate rupture potential of abdominal aortic aneurysm: identification of a finite strain constitutive model and evaluation of its applicability. Journal of Biomechanics, 33:475-482, 2000.

[183] P. Reymond, F. Merenda, F.Perren, D. Rufenacht, and N. Stergiopulos. Validation of a onedimensional model of the systemic arterial tree. Am J Physiol Heart Circ Physiol, 297:H208H222, 2009.

[184] A. M Robertson and A. Sequeira. A director theory approach for modeling blood flow in the arterial system: an alternative to classical 1d models. Mathematical Models and Methods in Applied Sciences, 15(06):871-906, 2005. 
[185] A.M. Robertson, A. Sequeira, and R.G. Owens. Rheological models for blood. In Cardiovascular mathematics, edited by A. Quarteroni, L. Formaggia, A. Veneziani, Chapter 6, pages 211-241. Springer, 2009.

[186] W. Rudin. Real and complex analysis. Tata McGraw-Hill Education, 1987.

[187] K. Sagawa. The ventricular pressure-volume diagram revisited. Circulation Research, 43(5):677-687, 1978.

[188] S. Salsa. Partial differential equations in action, From Modelling to Theory. Springer, Milan, 2008.

[189] O. Sander and A. Schiela. Variational analysis of the coupling between a geometrically exact cosserat rod and an elastic continuum. Zeitschrift fur Angewandte Mathematik und Physik, 65(6):1261-1288, 2013. Sandia National Laboratories Report SAND 2005-7817J.

[190] S.J. Savader, G.B. Lund, and F.A. Osterman. Volumetric evaluation of blood flow in normal renal arteries with a doppler flow wire: A feasibility study. J Vasc Interventional Radiol, 8(2):209-214, 1997.

[191] D. Schiavazzi and A. Mardsen. Data assimilation and propagation of uncertainty in multiscale cardiovascular simulation. Bulletin of the American Physical Society, 60(21):L24.00007, 2015.

[192] P. Segers, N. Stergiopulos, P. Verdonck, and R. Verhoeven. Assessment of distributed arterial network models. Medical and Biological Engineering and Computing, 35(6):729-736, 1997.

[193] S.J. Sherwin, L. Formaggia, J. Peiró, and V. Franke. Computational modelling of 1d blood ow with variable mechanical properties and its application to the simulation of wave propagation in the human arterial system. Int J Num Meth Fluids, 43:673-700, 2003.

[194] S.J. Sherwin, V. Franke, J. Peiró, and K. Parker. One-dimensional modelling of a vascular network in space-time variables. Journal of Engineering Mathematics, 47(3-4):217-259, 2003.

[195] J. Slawinski, T. Passerini, U. Villa, A. Veneziani, and V. Sunderam. Experiences with target-platform heterogeneity in clouds, grids, and on-premises resources. In Parallel and Distributed Processing Symposium Workshops \& PhD Forum (IPDPSW), 2012 IEEE 26th International, pages 41-52. IEEE, 2012.

[196] J. Slawinski, U. Villa, T. Passerini, A. Veneziani, and V. Sunderam. Issues in communication heterogeneity for message-passing concurrent computing. In Parallel and Distributed Processing Symposium Workshops 83 PhD Forum (IPDPSW), 2013 IEEE 27th International, pages 93-102. IEEE, 2013.

[197] B.N. Steele, J. Wan, J.P. Ku, T.J.R Hughes, and C.A. Taylor. In vivo validation of a onedimensional finite-element method for predicting blood flow in cardiovascular bypass grafts. IEEE Trans Biomed Eng, 50:649-656, 2003.

[198] N. Stergiopulos, B.E. Westerhof, J.J. Meister, and N. Westerhof. The four-element windkessel model. In Engineering in Medicine and Biology Society, 1996. Bridging Disciplines for Biomedicine. Proceedings of the 18th Annual International Conference of the IEEE, volume 4, pages 1715-1716, Oct 1996. 
[199] N. Stergiopulos, B.E. Westerhof, and N. Westerhof. Total arterial inertance as the fourth element of the windkessel model. American Journal of Physiology-Heart and Circulatory Physiology, 276(1):H81-H88, 1999.

[200] C.A. Taylor, T.J.R. Hughes, and C.K. Zarins. Finite element analysis of pulsatile flow in the abdominal aorta under resting and exercise conditions. American Society of Mechanical Engineers, Bioengineering Division, 33:81-82, 1996.

[201] C.A. Taylor, T.J.R. Hughes, and C.K. Zarins. Finite element modeling of blood flow in arteries. Computer Methods in Applied Mechanics and Engineering, 158(1-2):155-196, 1998.

[202] C.A. Taylor, D. Parker, T.J.R. Hughes, and C.K. Zarins. Finite element analysis of pulsatile flow in the human abdominal aorta: Geometric model construction from spiral ct data. American Society of Mechanical Engineers, Bioengineering Division, 35:361-362, 1997.

[203] T.E. Tezduyar, S. Sathe, T. Cragin, B. Nanna, B.S. Conklin, J. Pausewang, and M. Schwaab. Modelling of fluid-structure interactions with the space-time finite elements: arterial fluid mechanics. Int. J. Num. Methods Fluids, 54:901-922, 2007.

[204] K.W. Thompson. Time dependent boundary conditions for hyperbolic systems. Journal of computational physics, 68(1):1-24, 1987.

[205] R. Torii, M. Oshima, T. Kobayashi, K. Takagi, and T.E. Tezduyar. Role of Od peripheral vasculature model in fluidstructure interaction modeling of aneurysms. Computational Mechanics, 46(1):43-52, 2010.

[206] E.F. Toro. Brain venous haemodynamics, neurological diseases and mathematical modelling. a review. Applied Mathematics and Computation, page In press, 2015.

[207] E.F. Toro and A. Siviglia. Flow in collapsible tubes with discontinuous mechanical properties: mathematical model and exact solutions. Communications in Computational Physics, 13(2):361-385, 2013.

[208] A. Veneziani. The future of coronary stenting, A mathematical view, volume Coronary Stents, Innovations in 2015.

[209] A. Veneziani. Boundary conditions for blood flow problems. Proceedings of ENUMATH, Rannacher et al. eds., World Sci. Publishing, River Edge, NJ, 1998.

[210] A. Veneziani. Mathematical and Numerical Modeling of Blood Flow Problems. PhD thesis, University of Milan, 1998.

[211] A. Veneziani and C. Vergara. Flow rate defective boundary conditions in haemodinamics simulations. Int. J. Num. Methods Fluids, 47:803-816, 2005.

[212] A. Veneziani and C. Vergara. An approximate method for solving incompressible NavierStokes problems with flow rate conditions. Comput. Methods Appl. Mech. Engrg., 196(912):1685-1700, 2007. 
[213] A. Veneziani and C. Vergara. Inverse problems in cardiovascular mathematics: toward patient-specific data assimilation and optimization. International journal for numerical methods in biomedical engineering, 29(7):723-725, 2013.

[214] C. Vergara. Nitsche's method for defective boundary value problems in incompressibile fluiddynamics. J Sci Comp, 46(1):100-123, 2011.

[215] C. Vergara, M. Lange, S. Palamara, T. Lassila, A.F. Frangi, and A. Quarteroni. A coupled 3d-1d numerical monodomain solver for cardiac electrical activation in the myocardium with detailed purkinje network. J Comp Phys, in press, 2015.

[216] C. Vergara, R. Ponzini, A. Veneziani, and A. Redaelli D. Neglia O. Parodi. Womersley number-based estimation of flow rate with doppler ultrasound: Sensitivity analysis and first clinical application. Computer Methods and Programs in Biomedicine, 98(2):151-160, 2010.

[217] C. Vergara, F. Viscardi, L. Antiga, and G.B. Luciani. Influence of bicuspid valve geometry on ascending aortic fluid-dynamics: a parametric study. Artificial Organs, 36(4):368-378, 2012 .

[218] C. Vergara and P. Zunino. Multiscale modeling and simulation of drug release from cardiovascular stents. Multiscale Modeling and Simulation, 7(2):565-588, 2008.

[219] I.E. Vignon-Clementel, C.A. Figueroa, K. Jansen, and C. Taylor. Outflow boundary conditions for three-dimensional finite element modeling of blood flow and pressure waves in arteries. Comput. Methods Appl. Mech. Engrg., 195:3776-3996, 2006.

[220] F. Viscardi, C. Vergara, L. Antiga, S. Merelli, A. Veneziani, G. Puppini, G. Faggian, A. Mazzucco, and G.B. Luciani. Comparative finite element model analysis of ascending aortic flow in bicuspid and tricuspid aortic valve. Artificial organs, 34(12):1114-20, 2010.

[221] J. Wan, B. Steele, S.A. Spicer, S. Strohband, T.J.R. Hughes G.R. Feijóo, and C.A. Taylor. A one-dimensional finite element method for simulation-based medical planning for cardiovascular disease. Computer Methods in Biomechanics and Biomedical Engineering, 5(3):195-206, 2002.

[222] N. Westerhof, F. Bosman, C.J. De Vries, and A. Noordergraaf. Analog studies of the human systemic arterial tree. J Biomech, 2:121-143, 1969.

[223] N. Westerhof, J.W. Lankhaar, and B.E. Westerhof. The arterial windkessel. Medical $\&$ biological engineering \&3 computing, 47(2):131-141, 2009.

[224] D. Xiu and S. Sherwin. Parametric uncertainty analysis of pulse wave propagation in a model of a human arterial network. Journal of Computational Physics, 226(2):1385-1407, 2007.

[225] Huanhuan Yang and Alessandro Veneziani. Estimation of cardiac conductivities in ventricular tissue by a variational approach. Inverse Problems, 31(11):115001, 2015.

[226] H. Zafar, F. Sharif, and M.J. Leahy. Measurement of the blood flow rate and velocity in coronary artery stenosis using intracoronary frequency domain optical coherence tomography: Validation against fractional flow reserve. IJC Heart $\mathscr{E}$ Vasculature, 5:68-71, 2014. 
[227] P. Zunino. Numerical approximation of incompressible flows with net flux defective boundary conditions by means of penalty technique. Comput. Methods Appl. Mech. Engrg., 198(3740):3026-3038, 2009. 